\title{
Connection Problems for Quantum Affine KZ Equations and Integrable Lattice Models
}

\author{
Jasper V. Stokman ${ }^{1,2}$ \\ 1 KdV Institute for Mathematics, University of Amsterdam, Science Park 105-107, 1098 XG Amsterdam, \\ The Netherlands. E-mail: j.v.stokman@uva.nl \\ 2 IMAPP, Radboud University, Heyendaalseweg 135, 6525 AJ Nijmegen, The Netherlands
}

Received: 10 November 2014 / Accepted: 26 December 2014

Published online: 13 May 2015 - (C) The Author(s) 2015. This article is published with open access at Springerlink.com

\begin{abstract}
Cherednik attached to an affine Hecke algebra module a compatible system of difference equations, called quantum affine Knizhnik-Zamolodchikov (KZ) equations. In the case of a principal series module, we construct a basis of power series solutions of the quantum affine KZ equations. Relating the bases for different asymptotic sectors gives rise to a Weyl group cocycle, which we compute explicitly in terms of theta functions.

For the spin representation of the affine Hecke algebra of type $C$, the quantum affine $\mathrm{KZ}$ equations become the boundary qKZ equations associated to the Heisenberg spin- $\frac{1}{2}$ XXZ chain. We show that in this special case the results lead to an explicit 4-parameter family of elliptic solutions of the dynamical reflection equation associated to Baxter's 8vertex face dynamical $R$-matrix. We use these solutions to define an explicit 9-parameter elliptic family of boundary quantum Knizhnik-Zamolodchikov-Bernard (KZB) equations.
\end{abstract}

\section{Contents}

1. Introduction . . . . . . . . . . . . . . . . . . 1364

2. The Connection Problem for the Spin- $\frac{1}{2}$ XXZ Boundary qKZ Equations . 1366

2.1 The spin representation . . . . . . . . . . . . . . . . . . . . 1366

2.2 The boundary qKZ equations . . . . . . . . . . . . . . . . . . . 1369

2.3 basis consisting of power series solutions . . . . . . . . . . . . 1371

2.4 The connection problem . . . . . . . . . . . . . . . . . 1373

2.5 Dynamical $R$ - and $K$-matrices . . . . . . . . . . . . . . . . . . . 1374

2.6 Gauges of dynamical $R$ - and $K$-matrices . . . . . . . . . . . . . . . 1377

2.7 Face reformulation . . . . . . . . . . . . . . . . . 1380

2.8 Boundary qKZB equations . . . . . . . . . . . . . . . . . . 1383

3. The Connection Problem for Quantum Affine KZ Equations . . . . . . . . 1387

3.1 Notations . . . . . . . . . . . . . . . . . . . 1387 
3.2 Principal series representations . . . . . . . . . . . . . . . . . . . . . 1389

3.3 Quantum affine KZ equations . . . . . . . . . . . . . . . . . . . 1394

3.4 Power series solutions . . . . . . . . . . . . . . . . . . . . . . . . . . . 1395

3.5 The connection problem and its solution . . . . . . . . . . . 1398

3.6 The link to the spin- $-\frac{1}{2}$ XXZ boundary qKZ equations . . . . . . . 1403

References . . . . . . . . . . . . . . . . . . . . . . . 1407

\section{Introduction}

In Cherednik [10] associates to an abstract affine $R$-matrix $\left\{\mathcal{R}_{\alpha}\right\}_{\alpha}$, labelled by the roots $\alpha$ of an affine root system, a compatible system of equations called quantum affine $\mathrm{KZ}$ equations. For type A, affine $R$-matrices can be constructed using the braiding of quantum affine algebras. The resulting quantum affine $\mathrm{KZ}$ equations become the Frenkel-Reshetikhin-Smirnov qKZ equations [27,60]. For type C, affine $R$-matrices arise naturally in the context of integrable lattice models with boundaries. The corresponding quantum affine $\mathrm{KZ}$ equations are called boundary $\mathrm{qKZ}$ equations. In this case quantum affine symmetric pairs [41] produce examples, see, e.g., [4,13]. Besides the quantum group approach, which always leads to quantum affine $\mathrm{KZ}$ equations of classical type, one can attach quantum affine KZ equations to affine Hecke algebra modules. The associated affine $R$-matrices are constructed using the affine intertwiners of the double affine Hecke algebra [11].

In special cases the affine $R$-matrices can be obtained from both the quantum group and the Hecke algebra construction. The underlying actions of the quantum group and Hecke algebra are related by quantum Schur-Weyl type dualities.

In this paper we construct bases of power series solutions of quantum affine $\mathrm{KZ}$ equations associated to principal series modules of the affine Hecke algebra. The bases depend on asymptotic sectors, which are given in terms of Weyl chambers of the underlying root system. The connection matrices relating the bases for different asymptotic sectors give rise to a Weyl group cocycle. We solve the connection problem by deriving an explicit expression of the Weyl group cocycle in terms of theta functions. In addition we study the applications to integrable lattice models with boundaries in detail.

The connection to quantum integrable models is threefold. Firstly, for arbitrary root systems the difference Cherednik-Matsuo correspondence $[11,62,64]$ gives a bijective correspondence between the solutions of the quantum affine $\mathrm{KZ}$ equations associated to principal series modules and solutions of the spectral problem of RuijsenaarsMacdonald-Koornwinder-Cherednik (RMKC) difference operators. The RMKC difference operators are the Hamiltonians of the quantum relativistic trigonometric CalogeroMoser system, first introduced by Ruijsenaars [57] for type A. These quantum relativistic integrable models are intimately related to Macdonald-Koornwinder polynomials [9]. This point of view was emphasized in [64].

Secondly, special cases of the quantum affine $\mathrm{KZ}$ equations for principal series modules arise as compatibility conditions for correlation functions and form factors of (semi-)infinite XXZ spin chains [34,35,69]. In these cases vertex operator and algebraic Bethe ansatz techniques have been employed to construct solutions of the quantum affine $\mathrm{KZ}$ equations (see, e.g., $[27,34,54,68]$ and references therein for type A, and $[4,25,26,30,35,38,40,55,65,69]$ for type C). These methods have the drawback that they require additional assumptions on the associated XXZ spin chains, such as the existence of pseudo-vacuum vectors. Our construction of power series solutions of the quantum affine $\mathrm{KZ}$ equations is applicable without such restrictions. 
Thirdly, the solution of the connection problem is an explicit Weyl group cocycle expressed in terms of theta functions. For classical type and for special classes of principal series modules the cocycle gives rise to elliptic solutions of dynamical quantum YangBaxter and reflection equations.

In Sect. 3 we treat the general theory, but we start in Sect. 2 with a detailed discussion of the applications to integrable lattice models with boundaries. Applications to other classes of integrable lattice models, such as quasi-periodic lattice models with quantum supersymmetry or higher spin models, will be addressed in future work. The crucial step in applying the general theory to integrable lattice models is to decompose the affine Hecke algebra module underlying the pertinent integrable lattice model as a direct sum of principal series modules. For each principal series component the general theory from Sect. 3 can be applied to obtain an associated explicit connection cocycle of elliptic type.

We treat in full detail the spin representation of the affine Hecke algebra of type $\mathrm{C}$ $[29,65]$ encoding the affine Hecke algebra symmetries of the Heisenberg spin- $\frac{1}{2} \mathrm{XXZ}$ chain with arbitrary reflecting boundary conditions on both ends. The associated quantum affine KZ equations are boundary qKZ equations depending on 6 parameters: a representation parameter, a bulk parameter, and two free parameters for each end of the XXZ spin chain with reflecting ends. The associated $K$-matrices, encoding the integrability at the boundary, are those first obtained in [67] by direct computations. We explicitly apply the general theory from Sect. 3 to this context, resulting in the construction of bases of power series solutions of the boundary qKZ equations (Theorem 2.7) and the description of the associated connection matrices in terms of explicit elliptic solutions of dynamical quantum Yang-Baxter and reflection equations (Theorem 2.10). It leads to a 4-parameter family of solutions of the dynamical reflection equation with respect to Baxter's [5] dynamical elliptic $R$-matrix, with the representation parameter playing the role of the dynamical parameter. It can be reinterpreted as a 4-parameter family of solutions of the boundary Yang-Baxter equation for Baxter's eight vertex face model. Solutions to the boundary Yang-Baxter equations for the eight vertex face model, or equivalently, by vertex-face correspondences, to reflection equations for the eight vertex model itself, have been computed by direct computations before; see [32,33,67] from the vertex perspective, $[6,18,43]$ from the face perspective, and [31] for the explicit link by vertex-face correspondences.

The current paper thus provides a conceptual understanding of such solutions and of their free parameters. In fact, by the difference Cherednik-Matsuo correspondence $[11,62,65]$, the solutions of the boundary qKZ equations associated to the spin representation correspond to solutions of the spectral problem of the (higher order) Koornwinder operators [46] for a special family of spectral points which are naturally parametrized by the representation parameter of the spin representation. It results in the interpretation of the remaining 5 free parameters of the boundary $\mathrm{qKZ}$ equations as the free parameters in the theory of Koornwinder polynomials (see [65] for a detailed discussion). The four free parameters in the elliptic solutions of the dynamical reflection equation obtained from the computation of the associated connection problem then correspond to the Askey-Wilson parameters [64] in the theory of Koornwinder polynomials [46].

In $[19,21]$ solutions of dynamical quantum Yang-Baxter equations are used to define quantum analogs of the Knizhnik-Zamolodchikov-Bernard (KZB) equations. In the final part of Sect. 2 we construct quantum analogs of the KZB equations in the presence of boundaries. These boundary quantum KZB equations are defined in terms of solutions of the dynamical quantum Yang-Baxter equation and associated solutions of the left and right dynamical reflection equations. Applied to Baxter's elliptic dynamical 
R-matrix and the associated 4-parameter family of solutions of the dynamical reflection equation, we obtain an explicit 9-parameter elliptic family of boundary quantum Knizhnik-Zamolodchikov-Bernard (KZB) equations. We expect that a generalisation of the difference Cherednik-Matsuo correspondence relates their solutions to the solutions of the spectral problem of the 9-parameter family of the elliptic Ruijsenaars' systems of type $C$, as introduced by van Diejen [14] and Komori and Hikami [42].

In Sect. 3 we construct the bases of power series solutions of the quantum affine $\mathrm{KZ}$ equations associated to principal series modules. The explicit expressions of the associated connection matrices in terms of theta functions are derived using results of [64] (which dealt with the case of minimal principal series modules). In the last part of Sect. 3 we explain how these results, when applied to the spin representation of the affine Hecke algebra of type $C$, produce the results on integrable lattice models with boundaries as described in Sect. 2.

\section{The Connection Problem for the Spin- $\frac{1}{2}$ XXZ Boundary qKZ Equations}

2.1. The spin representation. Let $n \geq 2$ and $0<q<1$. We fix a basis $\left\{v_{+}, v_{-}\right\}$of $\mathbb{C}^{2}$ and represent linear operators on $\mathbb{C}^{2}$ and $\mathbb{C}^{2} \otimes \mathbb{C}^{2}$ as matrices with respect to the ordered basis $\left(v_{+}, v_{-}\right)$and $\left(v_{+} \otimes v_{+}, v_{+} \otimes v_{-}, v_{-} \otimes v_{+}, v_{-} \otimes v_{-}\right)$respectively.

Let $S_{n}$ be the symmetric group in $n$ letters and write $W_{0}=S_{n} \ltimes( \pm 1)^{n}$ for the hyperoctahedral group. Let $s_{1}, \ldots, s_{n}$ be simple reflections of $W_{0}$ satisfying $s_{i}^{2}=e$ and the braid relations $s_{i} s_{i+1} s_{i}=s_{i+1} s_{i} s_{i+1}(1 \leq i<n), s_{n-1} s_{n} s_{n-1} s_{n}=s_{n} s_{n-1} s_{n} s_{n-1}$ and $s_{i} s_{j}=s_{j} s_{i}$ if $|i-j|>1$, with $e$ the neutral element of $W_{0}$. The hyperoctahedral group $W_{0}$ acts on $\mathbb{C}^{n}$ by

$$
\begin{aligned}
& s_{i} \mathbf{z}=\left(z_{1}, \ldots, z_{i-1}, z_{i+1}, z_{i}, z_{i+2}, \ldots, z_{n}\right), \quad 1 \leq i<n, \\
& s_{n} \mathbf{z}=\left(z_{1}, \ldots, z_{n-1},-z_{n}\right),
\end{aligned}
$$

where $\mathbf{z}=\left(z_{1}, \ldots, z_{n}\right)$. Note that $\mathbb{Z}^{n}$ is $W_{0}$-stable.

The affine Weyl group of type $C_{n}$ is $W:=W_{0} \ltimes \mathbb{Z}^{n}$. We write $\lambda \mapsto \tau(\lambda)$ for the canonical group embedding $\mathbb{Z}^{n} \hookrightarrow W$. The action of $W_{0}$ on $\mathbb{Z}^{n}$ (resp. $\mathbb{C}^{n}$ ) extends to an action of $W$ by

$$
\tau(\lambda) \mathbf{z}:=\mathbf{z}+\lambda=\left(z_{1}+\lambda_{1}, \ldots, z_{n}+\lambda_{n}\right), \quad \lambda=\left(\lambda_{1}, \ldots, \lambda_{n}\right) \in \mathbb{Z}^{n} .
$$

Let $\left\{e_{i}\right\}_{i=1}^{n}$ be the standard orthonormal basis of $\mathbb{R}^{n}$ and set

$$
s_{0}:=\tau\left(e_{1}\right) s_{1} \cdots s_{n-1} s_{n} s_{n-1} \cdots s_{1} .
$$

Then

$$
s_{0} \mathbf{z}:=\left(1-z_{1}, z_{2}, \ldots, z_{n}\right)
$$

and $W$ is a Coxeter group with simple reflections $s_{0}, s_{1}, \ldots, s_{n}$. The associated Coxeter graph is

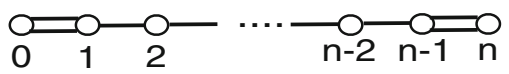

Note that

$$
\tau\left(e_{i}\right)=s_{i-1} \ldots s_{1} s_{0} s_{1} \ldots s_{n-1} s_{n} s_{n-1} \ldots s_{i}
$$

for $i=1, \ldots, n$. 
Fix parameters $\zeta^{\prime}, \kappa, \zeta \in \mathbb{C}$. The affine Hecke algebra $H=H\left(q^{\zeta^{\prime}}, q^{\kappa}, q^{\zeta}\right)$ of type $C_{n}$ is the unital associative algebra over $\mathbb{C}$ with generators $T_{0}, \ldots, T_{n}$ that satisfy the braid relations associated to the above Coxeter graph and satisfy the Hecke relations

$$
\begin{aligned}
\left(T_{0}-q^{-\zeta^{\prime}}\right)\left(T_{0}+q^{\zeta^{\prime}}\right) & =0, \\
\left(T_{i}-q^{-\kappa}\right)\left(T_{i}+q^{\kappa}\right) & =0, \quad 1 \leq i<n, \\
\left(T_{n}-q^{-\zeta}\right)\left(T_{n}+q^{\zeta}\right) & =0 .
\end{aligned}
$$

The spin representation $[29,65]$ of $H$ is defined as follows.

Proposition 2.1. There exists a unique representation $\pi_{\xi, \xi^{\prime}}^{s p}: H \rightarrow \operatorname{End}_{\mathbb{C}}\left(\left(\mathbb{C}^{2}\right)^{\otimes n}\right)$ such that

$$
\pi_{\xi, \xi^{\prime}}^{s p}\left(T_{i}\right)=\left(\begin{array}{cccc}
q^{-\kappa} & 0 & 0 & 0 \\
0 & 0 & 1 & 0 \\
0 & 1 & q^{-\kappa}-q^{\kappa} & 0 \\
0 & 0 & 0 & q^{-\kappa}
\end{array}\right)_{i, i+1}
$$

for $1 \leq i<n$ and

$$
\pi_{\xi, \xi^{\prime}}^{s p}\left(T_{0}\right)=\left(\begin{array}{cc}
q^{-\zeta^{\prime}}-q^{\zeta^{\prime}} & q^{-\xi^{\prime}} \\
q^{\xi^{\prime}} & 0
\end{array}\right)_{1}, \quad \pi_{\xi, \xi^{\prime}}^{s p}\left(T_{n}\right)=\left(\begin{array}{cc}
0 & q^{\xi} \\
q^{-\xi} & q^{-\zeta}-q^{\zeta}
\end{array}\right)_{n} .
$$

Here we have used the standard tensor leg notation to indicate on which tensor legs of the $n$-fold tensor product space $\left(\mathbb{C}^{2}\right)^{\otimes n}$ the matrices act.

The isomorphism class of $\pi_{\xi, \xi^{\prime}}^{s p}$ only depends on $\xi+\xi^{\prime}$, see [65, Prop. 3.5]. We write $\pi_{\left(\xi^{\prime}\right)}^{s p}$ for the representation $\pi_{0, \xi^{\prime}}^{s p}$, so that $\pi_{\xi, \xi^{\prime}}^{s p} \simeq \pi_{\left(\xi+\xi^{\prime}\right)}^{s p}$. We will sometimes suppress the representation parameter $\xi$ and write $\pi^{s p}=\pi_{(\xi)}^{s p}$.

Remark 2.2. The spin representation $\pi^{s p}$ factorizes through a representation of the twoboundary Temperley-Lieb algebra, see [29,65].

For generic parameters there are two natural complex linear bases of the representation space $\left(\mathbb{C}^{2}\right)^{\otimes n}$ which we denote by $\left\{v_{\epsilon}\right\}_{\epsilon}$ and $\left\{b_{\epsilon}\right\}_{\epsilon}$, where the indices $\underline{\epsilon}=\left(\epsilon_{1}, \epsilon_{2}, \ldots, \epsilon_{n}\right)$ are running over $\{ \pm 1\}^{n}$. The first basis $\left\{v_{\underline{\epsilon}}\right\}_{\underline{\epsilon}}$ is simply defined as

$$
\left(\mathbb{C}^{2}\right)^{\otimes n}=\bigoplus_{\underline{\epsilon}} \mathbb{C} v_{\underline{\epsilon}}, \quad v_{\underline{\epsilon}}:=v_{\epsilon_{1}} \otimes v_{\epsilon_{2}} \otimes \cdots \otimes v_{\epsilon_{n}},
$$

where $v_{ \pm 1}:=v_{ \pm}$. In particular, $v_{1}=v_{+}^{\otimes n}$ for $\underline{1}:=(1, \ldots, 1) \in\{ \pm 1\}^{n}$.

The basis elements $v_{\epsilon}$ can be expressed in terms of the $\pi^{s p}$-action of $H$ on the vector $v_{+}^{\otimes n}$ as follows. For any $w \in W$ and corresponding reduced expression $w=s_{i_{1}} s_{i_{2}} \cdots s_{i_{r}}$ $\left(0 \leq i_{j} \leq n\right)$ set

$$
T_{w}:=T_{i_{1}} T_{i_{2}} \cdots T_{i_{r}} \in H .
$$

The $T_{w} \in H$ are well defined (independent of the choice of reduced expression of $w \in W)$. Define $w_{\underline{\epsilon}} \in W_{0}$ for a $n$-tuple $\underline{\epsilon}=\left(\epsilon_{1}, \ldots, \epsilon_{n}\right) \in\{ \pm 1\}^{n}$ by

$$
w_{\underline{\epsilon}}:=\left(s_{i_{k}} s_{i_{k}+1} \cdots s_{n}\right) \cdots\left(s_{i_{2}} s_{i_{2}+1} \cdots s_{n}\right)\left(s_{i_{1}} s_{i_{1}+1} \cdots s_{n}\right)
$$


where $\left\{i_{1}, i_{2}, \ldots, i_{k}\right\}:=\left\{i \mid \epsilon_{i}=-1\right\}$ and $1 \leq i_{1}<i_{2}<\cdots<i_{k} \leq n$. Note that $w_{\epsilon}(1)=\underline{\epsilon}$ with respect to the action of $W_{0}$ on $\{ \pm 1\}^{n} \subset \mathbb{Z}^{n}$. The following lemma is now easy to verify.

Lemma 2.3. The elements $\left\{w_{\underline{\epsilon}}\right\}_{\underline{\epsilon}}$ are the minimal coset representatives of $W_{0} / S_{n}$, and

$$
v_{\underline{\epsilon}}=\pi^{s p}\left(T_{w_{\underline{\epsilon}}}\right) v_{+}^{\otimes n} .
$$

The second basis $\left\{b_{\underline{\epsilon}}\right\}_{\underline{\epsilon}}$ of $\left(\mathbb{C}^{2}\right)^{\otimes n}$ is defined as follows. Set for $i \in\{1, \ldots, n\}$,

$$
Y_{i}:=T_{i-1}^{-1} \cdots T_{1}^{-1} T_{0} T_{1} \cdots T_{n-1} T_{n} T_{n-1} \cdots T_{i} \in H
$$

(cf. (2.1)). Then $\left[Y_{i}, Y_{j}\right]=0$ and the elements $T_{1}, \ldots, T_{n}, Y_{1}^{ \pm 1}, \ldots, Y_{n}^{ \pm 1}$ algebraically generate $H$. The affine Hecke algebra can be completely characterised in terms of these generators, leading to the Bernstein-Zelevinsky presentation of $H$ (see [49]). We write $Y^{\lambda}=Y_{1}^{\lambda_{1}} Y_{2}^{\lambda_{2}} \ldots Y_{n}^{\lambda_{n}}$ for $\lambda=\left(\lambda_{1}, \ldots, \lambda_{n}\right) \in \mathbb{Z}^{n}$.

For $\eta=\left(\eta_{1}, \eta_{2}, \ldots, \eta_{n}\right) \in \mathbb{C}^{n}$ and a left $H$-module $V$ set

$$
\begin{aligned}
V_{\eta} & :=\left\{v \in V \mid Y_{i} v=q^{-\eta_{i}} v \quad \forall i\right\} \\
& =\left\{v \in V \mid Y^{\lambda} v=q^{-(\lambda, \eta)} v \quad \forall \lambda \in \mathbb{Z}^{n}\right\}
\end{aligned}
$$

for the common eigenspace of the commuting operators $Y_{i}$ on $V$ with eigenvalues $q^{-\eta_{i}}$, where we have used the standard bilinear form $(\mathbf{z}, \mathbf{w})=\sum_{i=1}^{n} z_{i} w_{i}$ on $\mathbb{C}^{n}$.

Set

$$
\gamma:=(\xi+(n-1) \kappa, \xi+(n-3) \kappa, \ldots, \xi+(1-n) \kappa) \in \mathbb{C}^{n} .
$$

It follows from the fact that $\left(\mathbb{C}^{2}\right)^{\otimes n}$ is a principal series module of $H$ with central character $q^{-\gamma}$ (see [65, Prop. 3.5] and Sect. 3.6) that the spin representation $\left(\pi_{(\xi)}^{s p},\left(\mathbb{C}^{2}\right)^{\otimes n}\right)$ decomposes for generic parameters as

$$
\left(\mathbb{C}^{2}\right)^{\otimes n}=\bigoplus_{\underline{\epsilon \in\{ \pm 1\}^{n}}}\left(\left(\mathbb{C}^{2}\right)^{\otimes n}\right)_{w_{\underline{\epsilon}} \gamma}
$$

with the common eigenspaces $\left(\left(\mathbb{C}^{2}\right)^{\otimes n}\right)_{w_{\epsilon} \gamma}$ being one-dimensional. The generic conditions on the parameters can be made precise, see [62, Prop. 2.12] and Sect. 3.2.

The basis element $b_{\underline{\epsilon}}$ is a particular choice of nonzero element from $\left(\left(\mathbb{C}^{2}\right)^{\otimes n}\right)_{w_{\underline{\epsilon}} \gamma}$. To define it we need to introduce a bit more notation. Let $R_{0}=\left\{ \pm e_{i} \pm e_{j}\right\}_{1 \leq i \neq j \leq n} \cup\left\{ \pm e_{i}\right\}_{i=1}^{n}$ be the root system of type $B_{n}$. We fix

$$
\left\{\alpha_{1}, \ldots, \alpha_{n-1}, \alpha_{n}\right\}=\left\{e_{1}-e_{2}, \ldots, e_{n-1}-e_{n}, e_{n}\right\}
$$

as basis of $R_{0}$ and write $R_{0}^{+}$(respectively $R_{0}^{-}$) for the corresponding set of positive (respectively negative) roots in $R_{0}$. The simple reflections $s_{1}, \ldots, s_{n}$ of $W_{0}$ correspond with the reflections in the simple roots $\alpha_{1}, \ldots, \alpha_{n}$. Let $\leq$ be the associated Bruhat order on $W_{0}$. Set for roots $\alpha \in R_{0}$,

$$
N_{\alpha}(\mathbf{z}):= \begin{cases}\frac{\left(1-q^{(\alpha, \mathbf{z})}\right)}{q^{\kappa}\left(1-q^{-2 \kappa+(\alpha, \mathbf{z})}\right)} & \text { if }\|\alpha\|^{2}=2, \\ \frac{\left(1-q^{2(\alpha, \mathbf{z})}\right)}{q^{\zeta}\left(1-q^{-\zeta-\zeta^{\prime}+(\alpha, \mathbf{z})}\right)\left(1+q^{-\zeta+\zeta^{\prime}+(\alpha, \mathbf{z})}\right)} & \text { if }\|\alpha\|^{2}=1 .\end{cases}
$$

It is easy to check that if $\alpha \in R_{0}^{+} \cap w_{\epsilon}^{-1} R_{0}^{-}\left(\underline{\epsilon} \in\{ \pm 1\}^{n}\right)$ then $\mathbf{z} \mapsto N_{\alpha}(\mathbf{z})$ is regular at $\mathbf{z}=\gamma$ (see also Sect. 3.2). The following lemma follows from standard techniques involving affine Hecke algebra intertwiners (see [62, Sect. 2.5] and Sect. 3.2). 
Lemma 2.4. Fix generic parameters and fix $\underline{\epsilon} \in\{ \pm 1\}^{n}$. There exists a unique $0 \neq b_{\underline{\epsilon}} \in$ $\left(\left(\mathbb{C}^{2}\right)^{\otimes n}\right)_{w_{\underline{\epsilon}} \gamma}$ satisfying

$$
b_{\underline{\epsilon}}=N_{\underline{\epsilon}} v_{\underline{\epsilon}}+\sum_{\underline{\epsilon^{\prime} \in\{ \pm 1\}^{n}: w_{\underline{\epsilon^{\prime}}}<w_{\underline{\epsilon}}}} L_{\underline{\epsilon^{\prime}}} v_{\underline{\epsilon}^{\prime}}
$$

for certain coefficients $L_{\underline{\epsilon}^{\prime}} \in \mathbb{C}$, where

$$
N_{\underline{\epsilon}}:=\prod_{\alpha \in R_{0}^{+} \cap w_{\underline{\epsilon}}^{-1} R_{0}^{-}} N_{\alpha}(\gamma) .
$$

The vectors $b_{\underline{\epsilon}}\left(\underline{\epsilon} \in\{ \pm 1\}^{n}\right)$ forms a basis of $\left(\mathbb{C}^{2}\right)^{\otimes n}$.

Note that $b_{1}=v_{+}^{\otimes n}=v_{\underline{1}}$.

2.2. The boundary $q K Z$ equations. The Baxterization [65, Sect. 4] of the representation $\pi^{s p}$ gives rise to a $W$-cocycle $\left\{C^{u}(\mathbf{z})\right\}_{u \in W}$ depending on two additional parameters $v$ and $v^{\prime}$, which are the two additional degrees of freedom in the $C^{\vee} C_{n}$ type double affine Hecke algebra $\mathbb{H}$ containing $H$ as a subalgebra. We do not recall here this construction of the cocycle $\left\{C^{u}(\mathbf{z})\right\}_{u \in W}$, which uses the intertwiners of the double affine Hecke algebra $\mathbb{H}$, but instead will give the resulting explicit formulas for $C^{u}(\mathbf{z})$ directly. Details on this Baxterization procedure can be found in $[9,62,65]$.

The values $C^{u}(\mathbf{z})$ are $\operatorname{End}_{\mathbb{C}}\left(\left(\mathbb{C}^{2}\right)^{\otimes n}\right)$-valued meromorphic functions in $\mathbf{z} \in \mathbb{C}^{n}$ satisfying

$$
C^{e}(\mathbf{z})=\operatorname{Id}_{\left(\mathbb{C}^{2}\right)^{\otimes n}}, \quad C^{u v}(\mathbf{z})=C^{u}(\mathbf{z}) C^{v}\left(u^{-1} \mathbf{z}\right)
$$

for all $u, v \in W$ and satisfying

$$
\begin{aligned}
& C^{s_{0}}(\mathbf{z})=\underline{\mathcal{K}}_{1}\left(\frac{1}{2}-z_{1}\right), \\
& C^{s_{i}}(\mathbf{z})=P_{i, i+1} \mathcal{R}_{i, i+1}\left(z_{i}-z_{i+1}\right), \quad 1 \leq i<n, \\
& C^{s_{n}}(\mathbf{z})=\mathcal{K}_{n}\left(z_{n}\right)
\end{aligned}
$$

with

$$
\mathcal{R}(z):=\frac{1}{1-q^{-2 \kappa+z}}\left(\begin{array}{cccc}
1-q^{-2 \kappa+z} & 0 & 0 & 0 \\
0 & q^{-\kappa}\left(1-q^{z}\right) & 1-q^{-2 \kappa} & 0 \\
0 & \left(1-q^{-2 \kappa}\right) q^{z} & q^{-\kappa}\left(1-q^{z}\right) & 0 \\
0 & 0 & 0 & 1-q^{-2 \kappa+z}
\end{array}\right)
$$

and

$$
\begin{aligned}
& \underline{\mathcal{K}}(z)=\underline{k}(z)\left(\begin{array}{cc}
\left(q^{\zeta^{\prime}}-q^{-\zeta^{\prime}}\right) q^{2 z}+\left(q^{v^{\prime}}-q^{-v^{\prime}}\right) q^{z} & q^{-\xi}\left(1-q^{2 z}\right) \\
q^{\xi}\left(1-q^{2 z}\right) & q^{\zeta^{\prime}}-q^{-\zeta^{\prime}}+\left(q^{v^{\prime}}-q^{-v^{\prime}}\right) q^{z}
\end{array}\right), \\
& \mathcal{K}(z)=k(z)\left(\begin{array}{cc}
q^{\zeta}-q^{-\zeta}+\left(q^{v}-q^{-v}\right) q^{z} & 1-q^{2 z} \\
1-q^{2 z} & \left(q^{\zeta}-q^{-\zeta}\right) q^{2 z}+\left(q^{v}-q^{-v}\right) q^{z}
\end{array}\right)
\end{aligned}
$$


and scalar functions $\underline{k}(z)$ and $k(z)$ given by

$$
\underline{k}(z):=\frac{q^{-\zeta^{\prime}}}{\left(1-q^{-\zeta^{\prime}-v^{\prime}+z}\right)\left(1+q^{-\zeta^{\prime}+v^{\prime}+z}\right)}, \quad k(z):=\frac{q^{-\zeta}}{\left(1-q^{-\zeta-v+z}\right)\left(1+q^{-\zeta+v+z}\right)},
$$

see [65, Sect. 4]. The fact that $\left\{C^{u}(\mathbf{z})\right\}_{u \in W}$ is a $W$-cocycle of the form (2.4) is equivalent to $\mathcal{R}(z)$ being a unitary $R$-matrix:

$$
\mathcal{R}_{12}\left(z_{1}-z_{2}\right) \mathcal{R}_{13}\left(z_{1}-z_{3}\right) \mathcal{R}_{23}\left(z_{2}-z_{3}\right)=\mathcal{R}_{23}\left(z_{2}-z_{3}\right) \mathcal{R}_{13}\left(z_{1}-z_{3}\right) \mathcal{R}_{12}\left(z_{1}-z_{2}\right),
$$

$\mathcal{R}_{21}(z) \mathcal{R}(-z)=\operatorname{Id}_{\mathbb{C}^{2} \otimes \mathbb{C}^{2}}$, and $\underline{\mathcal{K}}(z)$ respectively $\mathcal{K}(z)$ being a left respectively right unitary $K$-matrix with respect to $\mathcal{R}(z)$ :

$$
\begin{aligned}
& \mathcal{R}\left(z_{1}-z_{2}\right) \underline{\mathcal{K}}_{1}\left(z_{1}\right) \mathcal{R}_{21}\left(z_{1}+z_{2}\right) \underline{\mathcal{K}}_{2}\left(z_{2}\right)=\underline{\mathcal{K}}_{2}\left(z_{2}\right) \mathcal{R}\left(z_{1}+z_{2}\right) \underline{\mathcal{K}}_{1}\left(z_{1}\right) \mathcal{R}_{21}\left(z_{1}-z_{2}\right), \\
& \mathcal{R}_{21}\left(z_{1}-z_{2}\right) \mathcal{K}_{1}\left(z_{1}\right) \mathcal{R}\left(z_{1}+z_{2}\right) \mathcal{K}_{2}\left(z_{2}\right)=\mathcal{K}_{2}\left(z_{2}\right) \mathcal{R}_{21}\left(z_{1}+z_{2}\right) \mathcal{K}_{1}\left(z_{1}\right) \mathcal{R}\left(z_{1}-z_{2}\right),
\end{aligned}
$$

$\underline{\mathcal{K}}(z) \underline{\mathcal{K}}(-z)=\operatorname{Id}_{\mathbb{C}^{2}}=\mathcal{K}(z) \mathcal{K}(-z)$ (compare with [29, Sect. 2.3]). The equations (2.5) are called reflection equations, or boundary Yang-Baxter equations.

A transfer operator can be associated to the data $\underline{\mathcal{K}}(z), \mathcal{R}(z)$ and $\mathcal{K}(z)$ (see [59]). It is the transfer operator of the finite inhomogeneous XXZ spin- $\frac{1}{2}$ chain with arbitrary reflecting boundaries at both ends, see [24,29,65] and references therein.

In this paper we are interested in the quantum affine $\mathrm{KZ}$ equations related to the integrability data $\underline{\mathcal{K}}(z), \mathcal{R}(z)$ and $\mathcal{K}(z)$. They are the following boundary quantum $K Z$ equations (cf. $[10,65]$ and references therein).

Definition 2.5. Let $f: \mathbb{C}^{n} \rightarrow\left(\mathbb{C}^{2}\right)^{\otimes n}$ be a $\left(\mathbb{C}^{2}\right)^{\otimes n}$-valued meromorphic function on $\mathbb{C}^{n}$. We say that $f$ is a solution of the boundary quantum KZ equations associated to the spin representation $\pi^{s p}=\pi_{(\xi)}^{s p}$ if $f$ satisfies the difference equations

$$
C^{\tau(\lambda)}(\mathbf{z}) f(\mathbf{z}-\lambda)=f(\mathbf{z}) \quad \forall \lambda \in \mathbb{Z}^{n} .
$$

We write $\operatorname{Sol}_{s p}$ for the space of meromorphic solutions $f: \mathbb{C}^{n} \rightarrow\left(\mathbb{C}^{2}\right)^{\otimes n}$ of (2.6).

Remark 2.6. (i) By the cocycle property of $\left\{C^{v}(\mathbf{z})\right\}_{v \in W}$ we obtain a $W$-action on the space of $\left(\mathbb{C}^{2}\right)^{\otimes n}$-valued meromorphic functions on $\mathbb{C}^{n}$ by

$$
(\nabla(v) f)(\mathbf{z}):=C^{v}(\mathbf{z}) f\left(v^{-1} \mathbf{z}\right), \quad v \in W .
$$

The boundary quantum KZ equations (2.6) are equivalent to the equations $\nabla(\tau(\lambda)) f=f$ for all $\lambda \in \mathbb{Z}^{n}$. The solution space $\operatorname{Sol}_{s p}$ becomes a $W_{0}$-module by restricting the $\nabla$-action of $W_{0}$ to $\mathrm{Sol}_{s p}$.

(ii) The boundary quantum $\mathrm{KZ}$ equations (2.6) are equivalent to the equations $\nabla\left(\tau\left(e_{i}\right)\right) f=f(i=1, \ldots, n)$ which, by the cocycle property of $\left\{C^{u}(\mathbf{z})\right\}_{u \in W}$, (2.4) and (2.1), take on the explicit form

$$
C^{\tau\left(-e_{i}\right)}(\mathbf{z}) f\left(\mathbf{z}+e_{i}\right)=f(\mathbf{z}), \quad i=1, \ldots, n
$$

with

$$
\begin{aligned}
& C^{\tau\left(-e_{i}\right)}(\mathbf{z})=\mathcal{R}_{i+1, i}\left(z_{i}-z_{i+1}\right) \mathcal{R}_{i+2, i}\left(z_{i}-z_{i+2}\right) \cdots \mathcal{R}_{n i}\left(z_{i}-z_{n}\right) \mathcal{K}_{i}\left(z_{i}\right) \\
& \quad \times \mathcal{R}_{i n}\left(z_{i}+z_{n}\right) \cdots \mathcal{R}_{i, i+1}\left(z_{i}+z_{i+1}\right) \mathcal{R}_{i, i-1}\left(z_{i-1}+z_{i}\right) \cdots \mathcal{R}_{i 1}\left(z_{1}+z_{i}\right) \\
& \quad \times \underline{\mathcal{K}}_{i}\left(\frac{1}{2}+z_{i}\right) \mathcal{R}_{1 i}\left(1-z_{1}+z_{i}\right) \cdots \mathcal{R}_{i-2, i}\left(1-z_{i-2}+z_{i}\right) \mathcal{R}_{i-1, i}\left(1-z_{i-1}+z_{i}\right) .
\end{aligned}
$$


It is in this form that the boundary quantum $\mathrm{KZ}$ equations often appear in the literature, see, e.g., $[10,25,26,30,35,40,55,69]$.

(iii) For special choices of the $K$-matrices $\underline{\mathcal{K}}(z)$ and $\mathcal{K}(z)$ a vertex operator approach leads to solutions of the associated boundary quantum $\mathrm{KZ}$ equations [35]. These solutions give rise to correlation functions of the semi-infinite XXZ spin- $\frac{1}{2}$ chain. See $[4,25,26,38,65]$ for other constructions of solutions for special classes of $K$ matrices $\underline{\mathcal{K}}(z)$ and $\mathcal{K}(z)$.

2.3. basis consisting of power series solutions. Note that $\mathrm{Sol}_{s p}$ is a vector space over the field $F$ of scalar valued $\mathbb{Z}^{n}$-translation invariant meromorphic functions on $\mathbb{C}^{n}$. We now give, for generic parameters $\xi, \kappa, \zeta, \zeta^{\prime}, v, v^{\prime}$, the construction of a $F$-basis of $\operatorname{Sol}_{s p}$. It is a special case of the construction of a basis of solutions of the quantum affine $\mathrm{KZ}$ equations associated to principal series modules in Sect. 3.4 using the asymptotic techniques from $[50,51,63,64]$.

Let $w_{0} \in W_{0}$ for the longest Weyl group element $\left(w_{0}=-1\right.$ in the natural action of $W_{0}$ on $\mathbb{C}^{n}$ by permutations and sign changes) and define $\rho \in \mathbb{C}^{n}$ by

$$
\rho=\left(\zeta+\zeta^{\prime}+(n-1) \kappa, \zeta+\zeta^{\prime}+(n-3) \kappa, \ldots, \zeta+\zeta^{\prime}+(1-n) \kappa\right) .
$$

Let $\widetilde{\rho}$ be the vector $\rho$ with $v$ and $\zeta^{\prime}$ interchanged. The role of the plane wave in the asymptotic expansion is played by

$$
\mathcal{W}(\mathbf{z}, \mathbf{w}):=q^{\left(\rho-\mathbf{w}, \widetilde{\rho}+w_{0} \mathbf{z}\right)} .
$$

Let $\zeta, \zeta^{\prime}, v, v^{\prime} \in \mathbb{C}$. Define the associated Askey-Wilson parameters by

$$
\{a, b, c, d\}:=\left\{q^{\zeta+v},-q^{\zeta-v}, q^{\frac{1}{2}+\zeta^{\prime}+v^{\prime}},-q^{\frac{1}{2}+\zeta^{\prime}-v^{\prime}}\right\}
$$

and the dual Askey-Wilson parameters by

$$
\{\widetilde{a}, \widetilde{b}, \widetilde{c}, \widetilde{d}\}:=\left\{q^{\zeta+\zeta^{\prime}},-q^{\zeta-\zeta^{\prime}}, q^{\frac{1}{2}+v+v^{\prime}},-q^{\frac{1}{2}+v-v^{\prime}}\right\}
$$

cf. [64]. Note that the dual Askey-Wilson parameters are obtained from the AskeyWilson parameters by interchanging $v$ and $\zeta^{\prime}$. This defines an involution on the parameters, which we call the duality involution.

Write

$$
\left(x_{1}, \ldots, x_{m} ; q\right)_{\infty}:=\prod_{i=1}^{r}\left(x_{i} ; q\right)_{\infty}, \quad(x ; q)_{\infty}:=\prod_{j=0}^{\infty}\left(1-q^{j} x\right)
$$

for products of $q$-shifted factorials and set

$$
\begin{aligned}
\mathcal{S}_{s p}(\mathbf{z}):= & \prod_{i=1}^{n}\left(q^{1-z_{i}} / a, q^{1-z_{i}} / b, q^{1-z_{i}} / c, q^{1-z_{i}} / d ; q\right)_{\infty} \\
& \times \prod_{1 \leq r<s \leq n}\left(q^{1-2 \kappa-z_{r}+z_{s}}, q^{1-2 \kappa-z_{r}-z_{s}},-q^{1-z_{r}+z_{s}},-q^{1-z_{r}-z_{s}} ; q\right)_{\infty}
\end{aligned}
$$


and

$$
\begin{aligned}
\mathcal{U}(\mathbf{z}) & :=\frac{\mathcal{S}_{s p}(\mathbf{z})}{\prod_{i=1}^{n}\left(q^{1-2 z_{i}} ; q\right)_{\infty} \prod_{1 \leq r<s \leq n}\left(q^{2-2 z_{r}+2 z_{s}}, q^{2-2 z_{r}-2 z_{s}} ; q^{2}\right)_{\infty}} \\
& =\prod_{i=1}^{n} \frac{\left(q^{1-z_{i}} / a, q^{1-z_{i}} / b, q^{1-z_{i}} / c, q^{1-z_{i}} / d ; q\right)_{\infty}}{\left(q^{1-2 z_{i}} ; q\right)_{\infty}} \\
& \times \prod_{1 \leq r<s \leq n} \frac{\left(q^{1-2 \kappa-z_{r}+z_{s}}, q^{1-2 \kappa-z_{r}-z_{s}} ; q\right)_{\infty}}{\left(q^{1-z_{r}+z_{s}}, q^{1-z_{r}-z_{s}} ; q\right)_{\infty}} .
\end{aligned}
$$

Write $\tilde{\mathcal{U}}(\mathbf{z})$ for $\mathcal{U}(\mathbf{z})$ with $v$ and $\zeta^{\prime}$ interchanged (i.e. the Askey-Wilson parameters are replaced by the dual Askey-Wilson parameters). Write $Q_{+}$for the $\mathbb{Z}_{\geq 0}$-linear combinations of the simple roots $\alpha_{j}(1 \leq j \leq n)$.

Theorem 2.7. For generic parameter values we have

$$
\operatorname{Sol}_{s p}=\bigoplus_{\underline{\epsilon} \in\{ \pm 1\}^{n}} F \Phi_{\underline{\epsilon}}
$$

with $\Phi_{\epsilon} \in \operatorname{Sol}_{s p}$ characterised by the expansion formula

$$
\Phi_{\underline{\epsilon}}(\mathbf{z}):=\frac{\mathcal{W}\left(\mathbf{z}, w_{\underline{\epsilon}} \gamma\right)}{\mathcal{S}_{s p}(\mathbf{z}) \widetilde{\mathcal{U}}\left(w_{\underline{\epsilon}} \gamma\right)} \sum_{\alpha \in Q_{+}} \Gamma_{\alpha, \underline{\epsilon}} q^{-(\alpha, \mathbf{z})}, \quad \Gamma_{\alpha, \underline{\epsilon}} \in\left(\mathbb{C}^{2}\right)^{\otimes n}
$$

with the power series converging normally for $\mathbf{z}$ in compacta of $\mathbb{C}^{n}$, with $\gamma$ given by (2.3), and with leading coefficient given by

$$
\Gamma_{0, \underline{\epsilon}}:=\pi^{s p}\left(T_{w_{0}}\right) b_{\underline{\epsilon}} .
$$

The proof of the theorem is given in Sect. 3.6. It is obtained as a special case of Proposition 3.13, which deals with power series solutions of quantum affine KZ equations associated to principal series modules of affine Hecke algebras. Note that $\mathcal{S}_{s p}(\mathbf{z}) \Phi_{\epsilon}(\mathbf{z})$ is holomorphic in $\mathbf{z} \in \mathbb{C}^{n}$, i.e. the factor $\mathcal{S}_{s p}(\mathbf{z})$ in (2.8) is singling out the singularities of $\Phi_{\epsilon}(\mathbf{z})$.

Observe that $\Phi_{\underline{\epsilon}}(\mathbf{z})$ is the solution of the boundary qKZ equations which behaves as the "plane wave" $\left(\widetilde{\mathcal{U}}\left(w_{\epsilon} \gamma\right)^{-1} \pi^{s p}\left(T_{w_{0}}\right) b_{\epsilon}\right) \mathcal{W}\left(\mathbf{z}, w_{\epsilon} \gamma\right)$ when $\mathfrak{R}\left(\left(\alpha_{i}, \mathbf{z}\right)\right) \rightarrow-\infty$ for $i=1, \ldots, n$. This power series basis thus picks the Weyl chamber $C_{-}:=\{\mathbf{x} \in$ $\left.\mathbb{R}^{n} \mid\left(\alpha_{i}, \mathbf{x}\right)<0 \forall i\right\}$ as the asymptotic region in which the basis elements behave as plane waves.

Remark 2.8. (i) The generic conditions on the parameters can be made precise, see Sects. 3.4 and 3.6.

(ii) The choice of normalisation factor $\widetilde{\mathcal{U}}\left(w_{\epsilon} \gamma\right)^{-1}$ in (2.8) is motivated by duality properties of the asymptotic series solutions of quantum affine $\mathrm{KZ}$ equations associated to minimal principal series representations (see [50,51,63,64] and Sect. 3.4). 
2.4. The connection problem. In this subsection we assume that the parameters are generic. Let $v \in W_{0}$. Then $\left\{\left(\nabla(v) \Phi_{\underline{\epsilon}^{\prime}}\right)(\mathbf{z})\right\}_{\underline{\epsilon}^{\prime}}$ is a $F$-linear basis of $\operatorname{Sol}_{s p}$ with the basis elements behaving asymptotically as plane waves for $\mathfrak{R}\left(\left(\alpha_{i}, v^{-1} \mathbf{z}\right)\right) \rightarrow-\infty$ for $i=$ $1, \ldots, n$. Let $M_{c m ; \underline{\epsilon}, \underline{\epsilon}^{\prime}}^{v}(\cdot, \xi) \in F$ be the unique elements satisfying

$$
\left(\nabla(v) \Phi_{\underline{\epsilon}^{\prime}}\right)(\mathbf{z})=\sum_{\underline{\epsilon} \in\{ \pm 1\}^{n}} M_{c m ; \underline{\epsilon}, \underline{\epsilon}^{\prime}}^{v}(\mathbf{z}, \xi) \Phi_{\underline{\epsilon}}(\mathbf{z})
$$

(the subindex "cm" stands for "connection matrix"). We single out the dependence on the representation parameter $\xi$, because it will play the role of the dynamical parameter when relating the $M_{c m ; \epsilon, \epsilon^{\prime}}^{v}(\mathbf{z} ; \xi)$ to solutions of dynamical quantum Yang-Baxter and reflection equations.

Consider the corresponding $2^{n} \times 2^{n}$-matrix

$$
M_{c m}^{v}(\cdot, \xi)=\left(M_{c m ; \underline{\epsilon}, \underline{\epsilon}^{\prime}}^{v}(\cdot, \xi)\right)_{\underline{\epsilon}, \underline{\epsilon}^{\prime} \in\{ \pm 1\}^{n}}
$$

as an $\operatorname{End}_{\mathbb{C}}\left(\left(\mathbb{C}^{2}\right)^{\otimes n}\right)$-valued meromorphic function on $\mathbb{C}^{n}$ by

$$
M_{c m}^{v}(\mathbf{z}, \xi) v_{\underline{\epsilon}^{\prime}}:=\sum_{\underline{\epsilon} \in\{ \pm 1\}^{n}} M_{c m ; \underline{\epsilon}, \underline{\epsilon}^{\prime}}^{v}(\mathbf{z}, \xi) v_{\underline{\epsilon}} .
$$

Corollary 2.9. The set $\left\{M_{c m}^{v}(\mathbf{z}, \xi)\right\}_{v \in W_{0}}$ of $\operatorname{End}_{\mathbb{C}}\left(\left(\mathbb{C}^{2}\right)^{\otimes n}\right)$-valued meromorphic functions in $\mathbf{z} \in \mathbb{C}^{n}$ is a $W_{0}$-cocycle:

$$
M_{c m}^{e}(\mathbf{z}, \xi)=\operatorname{Id}_{\left(\mathbb{C}^{2}\right)^{\otimes n}}, \quad M_{c m}^{u v}(\mathbf{z}, \xi)=M_{c m}^{u}(\mathbf{z}, \xi) M_{c m}^{v}\left(u^{-1} \mathbf{z}, \xi\right)
$$

for all $u, v \in W_{0}$.

We call $\left\{M_{c m}^{v}(\mathbf{z}, \xi)\right\}_{v \in W_{0}}$ the connection cocycle of the boundary quantum KZ equations associated to the spin representation $\pi_{(\xi)}^{s p}$.

Let $h$ be the linear operator on $\mathbb{C}^{2}$ defined by $h v_{\epsilon}=\epsilon v_{\epsilon}(\epsilon \in\{ \pm 1\})$. Write $h_{i}$ for the linear operator on $\left(\mathbb{C}^{2}\right)^{\otimes n}$ given by

$$
h_{i}:=\mathrm{id}_{\left(\mathbb{C}^{2}\right)^{\otimes(i-1)}} \otimes h \otimes \mathrm{id}_{\left(\mathbb{C}^{2}\right) \otimes(n-i)} .
$$

For a family $S(\xi)$ of linear operators on $\left(\mathbb{C}^{2}\right)^{\otimes n}$ depending meromorphically on $\xi$ we write

$$
S\left(\xi+\alpha h_{i}\right) v_{\underline{\epsilon}}:=S\left(\xi+\alpha \epsilon_{i}\right) v_{\underline{\epsilon}} .
$$

for the associated linear operator on $\left(\mathbb{C}^{2}\right)^{\otimes n}$ in which the representation parameter $\xi$ is shifted according to the "weight" of the $i$ th tensor component of $v_{\underline{\epsilon}}$.

Write

$$
\theta\left(x_{1}, \ldots, x_{r} ; q\right):=\prod_{i=1}^{r} \theta\left(x_{i} ; q\right), \quad \theta(x ; q):=\prod_{j=0}^{\infty}\left(1-q^{j} x\right)\left(1-q^{j+1} / x\right)
$$

for products of renormalised Jacobi theta functions. Set

$$
\mathcal{C}(z, \xi):=\frac{\theta\left(\widetilde{a} q^{\xi}, \widetilde{b} q^{\xi}, \widetilde{c} q^{\xi}, d q^{\xi-z} / \widetilde{a} ; q\right)}{\theta\left(q^{2 \xi}, d q^{-z} ; q\right)} q^{-(\zeta+v-z)\left(\zeta+\zeta^{\prime}-\xi\right)}
$$


and write $\widetilde{\mathcal{C}}(z, \xi)$ for $\mathcal{C}(z, \xi)$ with $v$ and $\zeta^{\prime}$ interchanged,

$$
\widetilde{\mathcal{C}}(z, \xi):=\frac{\theta\left(a q^{\xi}, b q^{\xi}, c q^{\xi}, \widetilde{d} q^{\xi-z} / a ; q\right)}{\theta\left(q^{2 \xi}, \widetilde{d} q^{-z} ; q\right)} q^{-\left(\zeta+\zeta^{\prime}-z\right)(\zeta+v-\xi)}
$$

Note that $\mathcal{C}(z, \xi)$ is one-periodic in both $z$ and $\xi$. We show in Sect. 3.6 that the connection cocycle $\left\{M_{c m}^{u}(\mathbf{z}, \xi)\right\}_{u \in W_{0}}$ is characterised by the following formulas.

Theorem 2.10. We have

$$
\begin{aligned}
& M_{c m}^{s_{i}}(\mathbf{z}, \xi)=P_{i, i+1} R_{c m}\left(z_{i}-z_{i+1}, 2 \xi-2 \kappa\left(h_{1}+h_{2}+\cdots+h_{i-1}\right)\right)_{i, i+1}, \quad 1 \leq i<n, \\
& M_{c m}^{s_{n}}(\mathbf{z}, \xi)=K_{c m}\left(z_{n}, \xi-\kappa\left(h_{1}+h_{2}+\cdots+h_{n-1}\right)\right)_{n}
\end{aligned}
$$

where $P: \mathbb{C}^{2} \otimes \mathbb{C}^{2} \rightarrow \mathbb{C}^{2} \otimes \mathbb{C}^{2}$ is the permutation operator and

$$
\begin{aligned}
R_{c m}(z, \xi) & :=\left(\begin{array}{cccc}
1 & 0 & 0 & 0 \\
0 & A_{c m}(z, \xi) & B_{c m}(z, \xi) & 0 \\
0 & B_{c m}(z,-\xi) & A_{c m}(z,-\xi) & 0 \\
0 & 0 & 0 & 1
\end{array}\right) \\
K_{c m}(z, \xi) & :=\left(\begin{array}{ccc}
\alpha_{c m}(z, \xi) & \beta_{c m}(z, \xi) \\
\beta_{c m}(z,-\xi) & \alpha_{c m}(z,-\xi)
\end{array}\right)
\end{aligned}
$$

with the matrix coefficients given explicitly by

$A_{c m}(z, \xi):=\frac{\theta\left(q^{2 \kappa-\xi}, q^{-z} ; q\right)}{\theta\left(q^{2 \kappa-z}, q^{-\xi} ; q\right)} q^{2 \kappa(z-\xi)}, \quad B_{c m}(z, \xi):=\frac{\theta\left(q^{2 \kappa}, q^{-z-\xi} ; q\right)}{\theta\left(q^{-\xi}, q^{2 \kappa-z} ; q\right)} q^{(2 \kappa+\xi) z}$

and

$$
\alpha_{c m}(z, \xi):=\frac{\mathcal{C}(z, \xi)-\widetilde{\mathcal{C}}(\xi, z)}{\widetilde{\mathcal{C}}(\xi,-z)}, \quad \beta_{c m}(z, \xi):=\frac{\mathcal{C}(z, \xi)}{\widetilde{\mathcal{C}}(-\xi,-z)} .
$$

Note that $R_{c m}(z, \xi)$ and $K_{c m}(z, \xi)$ are one-periodic in both $z$ and $\xi$.

2.5. Dynamical $R$ - and $K$-matrices. The explicit form (2.10) of the connection cocycle implies that $R_{c m}(z, \xi)$ is a unitary solution of the dynamical quantum Yang-Baxter equation and $K_{c m}(z, \xi)$ a unitary solution of the dynamical reflection equation associated to $R_{c m}(z, \xi)$. Before stating the exact result we introduce the dynamical quantum YangBaxter and reflection equations in a slightly more general setting, replacing the spin space $\mathbb{C}^{2}$ by an arbitrary finite dimensional complex vector space $V$. Before doing so we need to extend some of the notations introduced in the previous paragraph to this more general context.

Let $h: V \rightarrow V$ be a semisimple complex linear operator. We write $V=\bigoplus_{\mu \in \mathbb{C}} V_{\mu}$ for the corresponding eigenspace decomposition, with $V_{\mu}$ the eigenspace of $h$ with eigenvalue $\mu$. Write $h_{i}: V^{\otimes n} \rightarrow V^{\otimes n}$ for the linear operator

$$
h_{i}:=\operatorname{Id}_{V}^{\otimes(i-1)} \otimes h \otimes \operatorname{Id}_{V}^{\otimes(n-i)} .
$$


We use the shorthand notation $\underline{\mu}:=\left(\mu_{1}, \ldots, \mu_{n}\right)$ for a $n$-tuple of complex numbers $\mu_{i}$. Set

$$
\left(V^{\otimes n}\right)_{\underline{\mu}}:=V_{\mu_{1}} \otimes \cdots \otimes V_{\mu_{n}}=\left\{v \in V^{\otimes n} \mid h_{i} v=\mu_{i} v, \quad i=1, \ldots, n\right\},
$$

so that $V^{\otimes n}=\bigoplus_{\mu}\left(V^{\otimes n}\right)_{\mu}$. Let $S(\xi)$ be a family of linear operator on $V^{\otimes n}$ depending meromorphically on a complex parameter $\xi \in \mathbb{C}$. For a given complex number $\alpha \in \mathbb{C}$ we write $\xi \mapsto S\left(\xi+\alpha h_{i}\right)$ for the family of linear operator on $V^{\otimes n}$ defined by

$$
S\left(\xi+\alpha h_{i}\right) v:=S\left(\xi+\alpha \mu_{i}\right) v, \quad v \in\left(V^{\otimes n}\right)_{\underline{\mu}} .
$$

We also occasionally use the backward weight shift. To define it, let $P_{\underline{\mu}}: V^{\otimes n} \rightarrow$ $\left(V^{\otimes n}\right)_{\underline{\mu}}$ be the projection along the direct sum decomposition $V^{\otimes n}=\bigoplus_{\underline{\nu}}\left(V^{\otimes n}\right)_{\underline{\nu}}$. Then we define

$$
S\left(\xi+\alpha \underline{h}_{i}\right):=\sum_{\underline{v}} P_{\underline{v}} S\left(\xi+\alpha v_{i}\right) .
$$

It is a linear operator on $V^{\otimes n}$ depending meromorphically on $\xi$.

A family $(z, \xi) \mapsto R(z, \xi)$ of linear operators on $V \otimes V$ depending meromorphically on two complex parameters $z$ and $\xi$ is called a dynamical $R$-matrix if $R(z, \xi)$ satisfies the dynamical quantum Yang-Baxter equation with spectral parameter [20]:

$$
\begin{aligned}
& R_{12}\left(z_{1}-z_{2}, \xi-2 \kappa h_{3}\right) R_{13}\left(z_{1}-z_{3}, \xi\right) R_{23}\left(z_{2}-z_{3}, \xi-2 \kappa h_{1}\right) \\
& =R_{23}\left(z_{2}-z_{3}, \xi\right) R_{13}\left(z_{1}-z_{3}, \xi-2 \kappa h_{2}\right) R_{12}\left(z_{1}-z_{2}, \xi\right)
\end{aligned}
$$

as a meromorphic family of linear operators on $V^{\otimes 3}$ (for later purposes it is convenient to add the factor two in the step size $2 \kappa$ ). The complex parameter $z$ is called the spectral parameter and $\xi$ the dynamical parameter. The dynamical quantum Yang-Baxter equation (2.11), also known as the Gervais-Neveu-Felder equation, first appeared in [28]. It is closely related to Baxter's star-triangle equation, see [20, Thm. 3] and Sect. 2.7.

We say that a dynamical $R$-matrix $R(z, \xi)$ satisfies the ice rule if

$$
[R(z, \xi), \Delta(h)]=0,
$$

where $\Delta(h):=h_{1}+h_{2}$. It is said to be unitarity if

$$
R_{21}(z, \xi) R(-z, \xi)=\mathrm{Id}_{V}^{\otimes 2} .
$$

We call $R(z, \xi)$ dynamically $P$-symmetric if

$$
R_{21}(z, \xi)=R(z,-\xi+2 \kappa \Delta(h))
$$

as linear operators on $V \otimes V$.

Remark 2.11. In [19] a family $\widetilde{R}(z, \xi)$ of linear operator on $V \otimes V$ is said to satisfy the dynamical quantum Yang-Baxter equation if

$$
\begin{aligned}
& \widetilde{R}_{12}\left(z_{1}-z_{2}, \xi-\kappa h_{3}\right) \widetilde{R}_{13}\left(z_{1}-z_{3} ; \xi+\kappa h_{2}\right) \widetilde{R}_{23}\left(z_{2}-z_{3} ; \xi-\kappa h_{1}\right) \\
& \quad=\widetilde{R}_{23}\left(z_{2}-z_{3} ; \xi+\kappa h_{1}\right) \widetilde{R}_{13}\left(z_{1}-z_{3} ; \xi-\kappa h_{2}\right) \widetilde{R}_{12}\left(z_{1}-z_{2} ; \xi+\kappa h_{3}\right) .
\end{aligned}
$$

The two versions (2.11) and (2.15) of the dynamical quantum Yang-Baxter equations are equivalent with the identification given by

$$
R(z, \xi)=\widetilde{R}(z, \xi-\kappa \Delta(h)),
$$

provided that $R(z, \xi)$ (hence also $\widetilde{R}(z, \xi))$ satisfies the ice-rule. 
Dynamical $R$-matrices encode the integrable structures underlying 2-dimensional face models from statistical mechanics with periodic boundary conditions (see, e.g., $[5,19,20])$. A well-known example is Baxter's 8-vertex face dynamical R-matrix. We come back to this example in Sect. 2.7.

Solutions of dynamical versions of reflection equations are related to integrable reflecting boundary conditions for face models, see, e.g., [2,6,7,23] and Sect. 2.7. In this paper we will use the following left and right version of the dynamical reflection equation.

Definition 2.12. Let $(z, \xi) \mapsto R(z, \xi)$ be a meromorphic family of linear operators on $V^{\otimes 2}$ of two complex parameters $z$ and $\xi$.

1. A meromorphic family $(z, \xi) \mapsto K(z, \xi)$ of linear operators on $V$ is called a right dynamical $K$-matrix with respect to $R(z, \xi)$ if $K(z, \xi)$ satisfies the right dynamical reflection equation

$$
\begin{aligned}
& R_{21}\left(z_{1}-z_{2}, 2 \xi\right) K_{1}\left(z_{1}, \xi-\kappa h_{2}\right) R_{12}\left(z_{1}+z_{2}, 2 \xi\right) K_{2}\left(z_{2}, \xi-\kappa h_{1}\right) \\
& \quad=K_{2}\left(z_{2}, \xi-\kappa h_{1}\right) R_{21}\left(z_{1}+z_{2}, 2 \xi\right) K_{1}\left(z_{1}, \xi-\kappa h_{2}\right) R_{12}\left(z_{1}-z_{2}, 2 \xi\right)
\end{aligned}
$$

as a family of linear operators on $V \otimes V$. The right dynamical $K$-matrix $K(z, \xi)$ is called unitary if

$$
K(z, \xi) K(-z, \xi)=\operatorname{Id}_{V} .
$$

2. A meromorphic family $(z, \xi) \mapsto K(z, \xi)$ of linear operators on $V$ is called a left dynamical $K$-matrix with respect to $R(z, \xi)$ if $\underline{K}(z, \xi)$ satisfies the left dynamical reflection equation

$$
\begin{aligned}
& R\left(z_{1}-z_{2}, 2 \xi+2 \kappa \Delta(h)\right) \underline{K}_{1}\left(z_{1}, \xi+\kappa h_{2}\right) R_{21}\left(z_{1}+z_{2}, 2 \xi+2 \kappa \Delta(h)\right) \underline{K}_{2}\left(z_{2}, \xi+\kappa h_{1}\right) \\
& =\underline{K}_{2}\left(z_{2}, \xi+\kappa h_{1}\right) R\left(z_{1}+z_{2}, 2 \xi+2 \kappa \Delta(h)\right) \underline{K}_{1}\left(z_{1}, \xi+\kappa h_{2}\right) R_{21}\left(z_{1}-z_{2}, 2 \xi+2 \kappa \Delta(h)\right)
\end{aligned}
$$

as a family of linear operators on $V \otimes V$. The left dynamical $K$-matrix $\underline{K}(z, \xi)$ is called unitary if $\underline{K}(z, \xi) \underline{K}(-z, \xi)=\operatorname{Id}_{V}$.

Remark 2.13. If $R(z, \xi)$ is dynamically $P$-symmetric and if $K(z, \xi)$ is a solution of the right dynamical reflection equation (2.16) then

$$
\underline{K}(z, \xi):=K(z,-\xi)
$$

is a solution of the left dynamical reflection equation (2.18) (and vice versa).

Let $R(z, \xi)$ be a linear operator on $V \otimes V$ and $K(z, \xi)$ a linear operator on $V$, both depending meromorphically on $(z, \xi) \in \mathbb{C}^{2}$. Let $P: V \otimes V \rightarrow V \otimes V$ be the permutation operator. Consider the linear operators

$$
\begin{aligned}
& M^{s_{i}}(\mathbf{z}, \xi):=P_{i, i+1} R_{i, i+1}\left(z_{i}-z_{i+1}, 2 \xi-2 \kappa\left(h_{1}+h_{2}+\cdots+h_{i-1}\right)\right), \quad 1 \leq i<n, \\
& M^{s_{n}}(\mathbf{z}, \xi):=K_{n}\left(z_{n}, \xi-\kappa\left(h_{1}+h_{2}+\cdots+h_{n-1}\right)\right) .
\end{aligned}
$$

on $V^{\otimes n}$, depending meromorphically on $(\mathbf{z}, \xi) \in \mathbb{C}^{n} \times \mathbb{C}$.

Proposition 2.14. Suppose that $R(z, \xi): V \otimes V \rightarrow V \otimes V$ satisfies the ice-rule (2.12). The following two statements are equivalent. 
(1) The linear operators (2.19) are part of a (necessarily unique) set $\left\{M^{u}(\mathbf{z}, \xi)\right\}_{u \in W_{0}}$ of $\operatorname{End}_{\mathbb{C}}\left(V^{\otimes n}\right)$-valued meromorphic functions $M^{u}(\mathbf{z}, \xi)$ in $(\mathbf{z}, \xi) \in \mathbb{C}^{n} \times \mathbb{C}$ satisfying the cocycle properties

$$
M^{e}(\mathbf{z}, \xi)=\operatorname{Id}_{V^{\otimes n}}, \quad M^{u v}(\mathbf{z}, \xi)=M^{u}(\mathbf{z}, \xi) M^{v}\left(u^{-1} \mathbf{z}, \xi\right)
$$

for all $u, v \in W_{0}$;

(2) $R(z, \xi)$ is a unitary dynamical $R$-matrix and $K(z, \xi)$ is an associated right unitary dynamical $K$-matrix.

Proof. This follows from a direct computation.

Consider now the special case that $V=\mathbb{C}^{2}$ with basis $\left\{v_{+}, v_{-}\right\}$and with $h$ the linear operator on $\mathbb{C}^{2}$ satisfying $h v_{\epsilon}=\epsilon v_{\epsilon}$. Since $R_{c m}(z, \xi)$ satisfies the ice-rule (2.12) we obtain from Corollary 2.9, Theorem 2.10 and Proposition 2.14 the following result.

Corollary 2.15. $R_{c m}(z, \xi)$ is a unitary dynamical $R$-matrix and $K_{c m}(z, \xi)$ is a right unitary dynamical $K$-matrix associated to $R_{c m}(z, \xi)$.

In the following subsection we will constructed gauges that transform $R_{c m}(z, \xi)$ into Baxter's [5] dynamical $R$-matrix associated to the eight vertex face model and $K_{c m}(z, \xi)$ into associated right dynamical $K$-matrices.

2.6. Gauges of dynamical $R$ - and $K$-matrices. We give two types of gauge transformations for a special class of dynamical $R$ - and $K$-matrices. We only consider the case that $V=\mathbb{C}^{2}$ and $h$ is the linear operator on $\mathbb{C}^{2}$ defined by $h v_{\epsilon}=\epsilon v_{\epsilon}$.

Proposition 2.16. Suppose that

$$
R(z, \xi):=\left(\begin{array}{cccc}
1 & 0 & 0 & 0 \\
0 & A(z, \xi) & B(z, \xi) & 0 \\
0 & B(z,-\xi) & A(z,-\xi) & 0 \\
0 & 0 & 0 & 1
\end{array}\right)
$$

is a unitary dynamical $R$-matrix and that

$$
K(z, \xi):=\left(\begin{array}{cc}
\alpha(z, \xi) & \beta(z, \xi) \\
\beta(z,-\xi) & \alpha(z,-\xi)
\end{array}\right)
$$

is a right unitary dynamical $K$-matrix associated to $R(z, \xi)$. Then

$$
R^{g}(z, \xi):=\left(\begin{array}{cccc}
1 & 0 & 0 & 0 \\
0 & A^{g}(z, \xi) & B^{g}(z, \xi) & 0 \\
0 & B^{g}(z,-\xi) & A^{g}(z,-\xi) & 0 \\
0 & 0 & 0 & 1
\end{array}\right)
$$

is a unitary dynamical $R$-matrix and

$$
K^{g}(z, \xi):=\left(\begin{array}{cc}
\alpha^{g}(z, \xi) & \beta^{g}(z, \xi) \\
\beta^{g}(z,-\xi) & \alpha^{g}(z,-\xi)
\end{array}\right)
$$

a right unitary dynamical $K$-matrix associated to $R^{g}(z, \xi)$ if 
(i) $A^{g}(z, \xi)=u(\xi) A(z, \xi), B^{g}(z, \xi)=B(z, \xi)$ and $K^{g}(z, \xi)=K(z, \xi)$ with $u(\xi) a$ meromorphic function satisfying $u(\xi) u(-\xi)=1$,

and if

(ii) $A^{g}(z, \xi)=q^{2 \kappa(\xi-z)} A(z, \xi), B^{g}(z, \xi)=q^{-z(2 \kappa+\xi)} B(z, \xi), \alpha^{g}(z, \xi)=q^{2 z \xi} \alpha(z, \xi)$ and $\beta^{g}(z, \xi)=\beta(z, \xi)$.

Proof. This follows by a tedious but direct computation.

Remark 2.17. The type (i) gauge transformations for dynamical $R$-matrices were considered before in [16].

Baxter's dynamical $R$-matrix $R_{B a}(z, \xi)$ corresponding to the eight vertex face model $[1,5]$ is defined as

$$
R_{B a}(z, \xi):=\left(\begin{array}{cccc}
1 & 0 & 0 & 0 \\
0 & A_{B a}(z, \xi) & B_{B a}(z, \xi) & 0 \\
0 & B_{B a}(z,-\xi) & A_{B a}(z,-\xi) & 0 \\
0 & 0 & 0 & 1
\end{array}\right)
$$

with the matrix coefficients given by

$$
A_{B a}(z, \xi):=\frac{\theta\left(q^{-z}, q^{2 \kappa+\xi} ; q\right)}{\theta\left(q^{2 \kappa-z}, q^{\xi} ; q\right)}, \quad B_{B a}(z, \xi):=\frac{\theta\left(q^{-z-\xi}, q^{2 \kappa} ; q\right)}{\theta\left(q^{2 \kappa-z}, q^{-\xi} ; q\right)} .
$$

Note that $R_{B a}(z, w)$ is unitary, satisfies the ice-rule and is dynamically $P$-symmetric. Write

$$
K_{B a}(z, \xi):=\left(\begin{array}{cc}
\alpha_{B a}(z, \xi) & \beta_{B a}(z, \xi) \\
\beta_{B a}(z,-\xi) & \alpha_{B a}(z,-\xi)
\end{array}\right)
$$

with

$$
\begin{aligned}
& \alpha_{B a}(z, \xi):=\alpha_{c m}(z, \xi) q^{2 z \xi}=\left(\frac{\mathcal{C}(z, \xi)-\widetilde{\mathcal{C}}(\xi, z)}{\widetilde{\mathcal{C}}(\xi,-z)}\right) q^{2 z \xi}, \\
& \beta_{B a}(z, \xi):=\beta_{c m}(z, \xi)=\frac{\mathcal{C}(z, \xi)}{\widetilde{\mathcal{C}}(-\xi,-z)}
\end{aligned}
$$

(recall the definition of $\mathcal{C}(z, \xi)$ from (2.9)). If we need to specify the dependence of $K_{B a}(z, \xi)$ on the parameters $\zeta, \zeta^{\prime}, v, v^{\prime}$ then we write $K_{B a}\left(z, \xi ; \zeta, \zeta^{\prime}, v, v^{\prime}\right)$.

Corollary 2.18. $R_{B a}(z, \xi)$ is a unitary dynamical $R$-matrix and $K_{B a}(z, \xi)$ is a unitary right dynamical $K$-matrix associated to $R_{B a}(z, \xi)$.

Proof. Apply to the unitary dynamical $R$-matrix $R_{c m}(z, \xi)$ and the associated unitary right dynamical $K$-matrix $K_{c m}(z, \xi)$ gauge (i) with

$$
u(\xi)=\frac{\theta\left(q^{2 \kappa+\xi}, q^{-\xi} ; q\right)}{\theta\left(q^{2 \kappa-\xi}, q^{\xi} ; q\right)}
$$

and apply subsequently gauge (ii). Then we obtain $R_{B a}(z, \xi)$ and $K_{B a}(z, \xi)$. Hence $R_{B a}(z, \xi)$ is a unitary dynamical $R$-matrix and $K_{B a}(z, \xi)$ an associated unitary right dynamical $K$-matrix as a consequence of Corollary 2.15. 
The fact that $R_{B a}(z, \xi)$ is a unitary solution of the dynamical quantum Yang-Baxter equation goes back to Baxter [5] (the dynamical quantum Yang-Baxter equation in [5] takes the form of star-triangle equations, see [19] and the next subsection for more details on this viewpoint). The interpretation of Baxter's solution $R_{B a}(z, \xi)$ in terms of connection matrices for the Frenkel-Reshetikhin-Smirnov qKZ equations goes back to [27] (see also [44,52]). Other examples of unitary dynamical $R$-matrices have been constructed in, e.g., $[12,36,37,44]$. Some of these examples are constructed using fusion techniques, others by computing connection matrices. A quantum group context for dynamical $R$-matrices was developed by Felder, Etingof and Varchenko, see, e.g., [16, 17,20].

Baxter's unitary dynamical $R$-matrix $R_{B a}(z, \xi)$ satisfies dynamical crossing symmetry. In coordinates it reads

$$
\delta_{2} \epsilon_{2} R_{\epsilon_{1},-\delta_{2}}^{\delta_{1},-\epsilon_{2}}(z, \xi)=q^{-\kappa\left(1+\epsilon_{2}\right)} \frac{\theta\left(q^{\xi+2 \kappa \epsilon_{2}}, q^{z} ; q\right)}{\theta\left(q^{\xi}, q^{z-2 \kappa} ; q\right)} R_{\delta_{1} \delta_{2}}^{\epsilon_{1} \epsilon_{2}}\left(2 \kappa-z, \xi+2 \kappa \epsilon_{2}\right),
$$

where we have written $R_{B a}(z, \xi) v_{\delta_{1}} \otimes v_{\delta_{2}}=\sum_{\epsilon_{1}, \epsilon_{2}} R_{\delta_{1} \delta_{2}}^{\epsilon_{1} \epsilon_{2}}(z, \xi) v_{\epsilon_{1}} \otimes v_{\epsilon_{2}}$. In coordinate free dynamical notations it reads

$$
\sigma_{2}^{y} R_{B a}(z, \xi)^{T_{1}} \sigma_{2}^{y}=q^{-\kappa\left(1+h_{2}\right)} \frac{\theta\left(q^{\xi+2 \kappa h_{2}}, q^{z} ; q\right)}{\theta\left(q^{\xi}, q^{z-2 \kappa} ; q\right)} R_{B a}\left(2 \kappa-z, \xi+2 \kappa \underline{h}_{2}\right)
$$

where $\sigma^{y}=\left(\begin{array}{cc}0 & -\sqrt{-1} \\ \sqrt{-1} & 0\end{array}\right)$ and $T_{1}$ is transposition in the first tensor component.

In Corollary 2.18 we have constructed a 4-parameter family of unitary right dynamical $K$-matrices associated to $R_{B a}(z, \xi)$ by relating them to connection matrices of boundary qKZ equations. Dynamical $K$-matrices associated to $R_{B a}(z, \xi)$ have been constructed before by direct computations in the study of the eight vertex solid-on-solid model with integrable reflecting boundaries (see $[32,33,67]$ from the vertex perspective, $[6,18,43]$ from the face perspective, and [31] for the explicit link by vertex-face correspondences). In particular, see [6, Sect. 4.3] for alternative parametrisations of unitary right dynamical $K$-matrices associated to $R_{B a}(z, \xi)$ depending on four parameters. We will explain the link to the eight vertex face model with integrable reflecting boundaries in more detail in the following subsection.

Remark 2.19. (i) Let $S$ be the spin-reversal operator on $\mathbb{C}^{2}$, defined as the linear map $S: \mathbb{C}^{2} \rightarrow \mathbb{C}^{2}$ satisfying $S\left(v_{+}\right)=v_{-}$and $S\left(v_{-}\right)=v_{+}$. Then $K_{B a}(z, w)$ has the spin-reversal symmetry

$$
S K_{B a}(z, \xi) S^{-1}=K_{B a}(z,-\xi) .
$$

(ii) The matrix coefficient $\beta_{B a}(z, \xi)$ of $K_{B a}(z, \xi)$ decouples,

$$
\beta_{B a}(z, \xi)=\frac{\widetilde{\mu}(\xi)}{\mu(-z)}
$$

with

$$
\mu(z):=\frac{\theta\left(a q^{z}, b q^{z}, c q^{z}, d q^{z} ; q\right)}{\theta\left(q^{2 z} ; q\right)} q^{2\left(\zeta+\zeta^{\prime}\right) z}
$$

and with $\widetilde{\mu}(z)$ obtained from $\mu(z)$ by interchanging $v$ and $\zeta^{\prime}$. The function $\mu(z)$ is a natural elliptic analog of the $c$-function associated to the Askey-Wilson 
polynomials [3]. It naturally appears in the study of the Askey-Wilson function transform (see [39,63, Lem. 4.4]), as well as in Rains' [53] difference operator acting on BC-symmetric theta functions, which plays an important role in the study of Rains' interpolation theta functions.

(iii) In [23] a reflection equation different from (2.16) is considered in relation to Baxter's dynamical $R$-matrix $R_{B a}(z, \xi)$. It does not involve shifts in the dynamical parameter. The one-parameter family of diagonal solutions from [23] appears to be unrelated to the four-parameter family of dynamical $K$-matrices $K_{B a}(z, \xi)$.

(iv) The dynamical $K$-matrix $K_{B a}(z, \xi)$ is anti-diagonal iff $\mathcal{C}(z, \xi)=\widetilde{\mathcal{C}}(\xi, z)$. An explicit discrete set of parameter values for which this is the case has been derived in $[61$, Sect. 3], see also [64]. In [61,64] the condition $\mathcal{C}(z, \xi)=\widetilde{\mathcal{C}}(\xi, z)$ is related to the theory of reflectionless Askey-Wilson second-order $q$-difference operators and to the theory of Baker-Akhiezer functions [8].

2.7. Face reformulation. Felder [20] observed that solutions of the dynamical quantum Yang-Baxter equation satisfying the ice-rule are in one-to-one correspondence to solutions of the star-triangle equation [5], see also [56, Sect. 3]. We recall it here, and extend the correspondence to associated dynamical $K$-matrices. We will match it with the notations from [6] in order to facilitate the comparison of our 4-parameter family of dynamical $K$-matrices from Corollary 2.18 with the 4-parameter family of solutions of the boundary Yang-Baxter equation from [6, Sect. 4.3].

In this subsection we fix a finite dimensional complex vector space $V$ and a semisimple linear operator $h$ with simple spectrum $I$ (in many examples $V$ is the vector space underlying a finite dimensional irreducible $\mathfrak{s l}_{2}(\mathbb{C})$-representation and $h$ is the Cartan generator of $\mathfrak{s l}_{2}(\mathbb{C})$, in which case $h$ acts semisimply on $V$ with simple spectrum given by $\{k-2 l\}_{l=0}^{k}$ for some $k \in \mathbb{N}$ ). We fix a linear basis $\left\{v_{\mu}\right\}_{\mu \in I}$ of $V$ satisfying $h v_{\mu}=\mu v_{\mu}$.

Suppose $R(z, \xi)$ is a linear operator on $V \otimes V$ depending meromorphically on $(z, \xi) \in$ $\mathbb{C}^{2}$ and write

$$
R(z, \xi) v_{\mu_{1}} \otimes v_{\mu_{2}}=\sum_{v_{1}, \nu_{2} \in I} R_{\mu_{1} \mu_{2}}^{v_{1} \nu_{2}}(z, \xi) v_{\nu_{1}} \otimes v_{v_{2}}
$$

for $\mu_{1}, \mu_{2} \in I$. For $\mu_{i}, v_{i} \in \mathbb{C}$ we set $R_{\mu_{1} \mu_{2}}^{v_{1} \nu_{2}}(z, \xi) \equiv 0$ if $\left(\mu_{1}, \mu_{2}, v_{1}, v_{2}\right) \notin I^{\times 4}$. Note that the ice rule (2.12) for $R(z, \xi)$ is equivalent to the property that $R_{\mu_{1} \mu_{2}}^{v_{1} \nu_{2}}(z, \xi) \equiv 0$ unless $\mu_{1}+\mu_{2}=v_{1}+v_{2}$. Set for $a, b \in \mathbb{C}$,

$$
A_{a b}:= \begin{cases}1 & \text { if } b-a \in I, \\ 0 & \text { if } b-a \notin I .\end{cases}
$$

Define for $a, b, c, d \in \mathbb{C}$ the Boltzmann weight

$$
W\left(\begin{array}{ll}
a & b \\
c & d
\end{array} \mid z, \xi\right):=R_{b-a, d-b}^{d-c, c-a}(z, \xi-2 \kappa a) .
$$

Note that $W\left(\begin{array}{ll}a & b \\ c & d\end{array} \mid z, \xi\right) \equiv 0$ unless $A_{a b} A_{a c} A_{b d} A_{c d}=1$. By [19, Sect. 5] (see also [56, Sect. 3]), we have the following result. 
Proposition 2.20. Suppose that $R(z, \xi): V \otimes V \rightarrow V \otimes V$ satisfies the ice rule (2.12). Then $R(z, \xi)$ is a solution of the dynamical quantum Yang-Baxter equation (2.11) if and only if the corresponding Boltzmann weights (2.25) satisfy the star triangle equations

$$
\begin{aligned}
& \sum_{g} W\left(\begin{array}{ll}
f & g \\
a & b
\end{array} \mid z_{1}-z_{2}, \xi\right) W\left(\begin{array}{ll}
g & d \\
b & c
\end{array} \mid z_{1}-z_{3}, \xi\right) W\left(\begin{array}{ll}
f & e \\
g & d
\end{array} \mid z_{2}-z_{3}, \xi\right) \\
& =\sum_{g} W\left(\begin{array}{ll}
a & g \\
b & c
\end{array} \mid z_{2}-z_{3}, \xi\right) W\left(\begin{array}{ll}
f & e \\
a & g
\end{array} \mid z_{1}-z_{3}, \xi\right) W\left(\begin{array}{ll}
e & d \\
g & c
\end{array} \mid z_{1}-z_{2}, \xi\right)
\end{aligned}
$$

for all $a, b, c, d, e, f \in \mathbb{C}$.

Remark 2.21. Note that (2.26) is a nontrivial identity only if $A_{a b} A_{b c} A_{d c} A_{e d} A_{f e} A_{f a}=$ 1. Furthermore, for fixed $a, b, c, d, e, d$ the left and right hand sides of (2.26) are well defined since the summand on the left (resp. right) is zero unless $g \in \mathbb{C}$ is in the finite (possibly empty) set of complex numbers $g$ satisfying $A_{f g} A_{g b} A_{g d}=1$ (resp. $\left.A_{a g} A_{e g} A_{g c}=1\right)$. With these two remarks it is clear that (2.26) coincides with the star triangle equations for face models, see, e.g., [6, (2.3)].

Unitarity (2.13) of $R(z, \xi)$ is equivalent to the inversion relation

$$
\sum_{e} W\left(\begin{array}{ll}
d & e \\
a & b
\end{array} \mid z, \xi\right) W\left(\begin{array}{ll}
d & c \\
e & b
\end{array} \mid-z, \xi\right)=\delta_{a c}
$$

with $\delta_{a c}$ the Kronecker delta function, cf. [6, (2.13)].

Boundary star-triangle equations are defined in $[6,7,48]$, leading to integrable face models with boundary. We now proceed to explain its equivalence to the right dynamical reflection equation (2.16). Let $K(z, \xi): V \rightarrow V$ be a linear operator depending meromorphically on $(z, \xi) \in \mathbb{C}^{2}$ and write

$$
K(z, \xi) v_{\mu}=\sum_{v \in I} K_{\mu}^{v}(z, \xi) v_{v}
$$

for $\mu \in I$. For $\mu, v \in \mathbb{C}$ we set $K_{\mu}^{\nu}(z, \xi) \equiv 0$ if $(\mu, v) \notin I \times I$. Define for $a, b, c \in \mathbb{C}$ the boundary Boltzmann weight

$$
B\left(\begin{array}{ll}
b & c \\
& a
\end{array} \mid z, \xi\right):=K_{c-b}^{a-b}\left(z, \frac{\xi}{2}-\kappa b\right) .
$$

Note that $B\left(\begin{array}{ll}b & c \\ & a\end{array} \mid z, \xi\right) \equiv 0$ unless $A_{b a} A_{b c}=1$. The following result can be checked by a direct computation.

Proposition 2.22. Assume that $R(z, \xi): V \otimes V \rightarrow V \otimes V$ is a dynamical $R$-matrix satisfying the ice rule (2.12). Define the associated Boltzmann weights by (2.25). Then $K(z, \xi)$ is a right dynamical $K$-matrix with respect to $R(z, \xi)$ if and only if the corresponding boundary Boltzmann weights (2.27) satisfy the boundary Yang-Baxter equations

$$
\begin{aligned}
& \sum_{f, g} W\left(\begin{array}{ll}
c & f \\
d & e
\end{array} \mid z_{1}-z_{2}, \xi\right) B\left(\begin{array}{c}
g \\
e
\end{array} \mid z_{1}, \xi\right) W\left(\begin{array}{ll}
c & b \\
f & g
\end{array} \mid z_{1}+z_{2}, \xi\right) B\left(\begin{array}{ll}
b & a \\
g
\end{array} \mid z_{2}, \xi\right) \\
& =\sum_{f, g} B\left(\begin{array}{l}
g \\
e
\end{array} \mid z_{2}, \xi\right) W\left(\begin{array}{cc}
c & f \\
d & g
\end{array} \mid z_{1}+z_{2}, \xi\right) B\left(\begin{array}{c}
a \\
f
\end{array} \mid z_{1}, \xi\right) W\left(\begin{array}{ll}
c & b \\
f & a
\end{array} \mid z_{1}-z_{2}, \xi\right)
\end{aligned}
$$


for all $a, b, c, d, e \in \mathbb{C}$.

By a similar reasoning as in Remark 2.21 one shows that the equations (2.28) are equivalent to the boundary Yang-Baxter equations [6, (2.4)]. Note that unitarity (2.17) of $K(z, \xi)$ is equivalent to the boundary inversion relation

$$
\sum_{d} B\left(\begin{array}{l}
d \\
c
\end{array} \mid z, \xi\right) B\left(\begin{array}{l}
a \\
d
\end{array} \mid-z, \xi\right)=\delta_{a c}
$$

cf. [6, (2.14)].

Solutions of the star triangle (2.26) and boundary Yang-Baxter equations (2.28) give rise to two-dimensional integrable face models with boundary. We refer to [6,7] and references therein for details. I will finish this subsection by giving the explicit formulas relating $R_{B a}(z, \xi)$ to the Boltzmann weights of the eight vertex face model and subsequently pushing the associated 4-parameter solutions of the right dynamical reflection equation (Corollary 2.18) to a 4-parameter family of solutions of the associated boundary Yang-Baxter equations. So in the remainder of this section we take $V=\mathbb{C}^{2}=$ $\mathbb{C} v_{+} \oplus \mathbb{C} v_{-}$and $h v_{ \pm}= \pm v_{ \pm}$, so that $I=\{-1,1\}$ and $A_{a b}=1$ iff $|a-b|=1$.

We gauge $R_{B a}(z, \xi)$ to the unitary dynamical $R$-matrix $R_{8 v S O S}(z, \xi)$ given by

$$
R_{8 v S O S}(z, \xi):=q^{\frac{z}{2}} \frac{\theta\left(q^{2 \kappa-z} ; q\right)}{\theta\left(q^{2 \kappa} ; q\right)}\left(\begin{array}{cccc}
1 & 0 & 0 & 0 \\
0 & u(\xi) A_{B a}(z, \xi) & B_{B a}(z, \xi) & 0 \\
0 & B_{B a}(z,-\xi) & u(-\xi) A_{B a}(z,-\xi) & 0 \\
0 & 0 & 0 & 1
\end{array}\right)
$$

with

$$
u(\xi):=q^{-\kappa}\left(\frac{\theta\left(q^{-\xi+2 \kappa} ; q\right)}{\theta\left(q^{-\xi-2 \kappa} ; q\right)}\right)^{\frac{1}{2}}
$$

Denote the corresponding Boltzmann weights (see (2.25)) by $W_{8 v S O S}\left(\begin{array}{ll}a & b \\ c & d\end{array} \mid z, \xi\right)$. Then the nonzero Boltzmann weights are

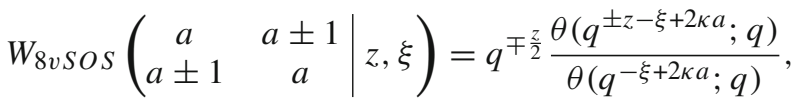

$$
\begin{aligned}
& W_{8 v \operatorname{SOS}}\left(\begin{array}{cc|c}
a \pm 1 & a \\
a & a \mp 1 & z, \xi
\end{array}\right)=q^{\frac{z}{2}} \frac{\theta\left(q^{2 \kappa-z} ; q\right)}{\theta\left(q^{2 \kappa} ; q\right)}, \\
& W_{8 v \operatorname{SOS}}\left(\begin{array}{cc}
a & a \pm 1 \\
a \mp 1 & a
\end{array} \mid z, \xi\right)=q^{\kappa}\left(\frac{\theta\left(q^{-\xi+2 \kappa(a-1)}, q^{-\xi+2 \kappa(a+1)} ; q\right)}{\theta\left(q^{-\xi+2 \kappa a} ; q\right)^{2}}\right)^{\frac{1}{2}} q^{\frac{z}{2}} \frac{\theta\left(q^{-z} ; q\right)}{\theta\left(q^{2 \kappa} ; q\right)},
\end{aligned}
$$

which coincide with the standard weights of the eight-vertex face model. To relate it to the notations in [6], note that it corresponds to the Boltzmann weights [6, (4.4)] by identifying the parameters $\lambda, w_{0}, u$ in $[6,(4.4)]$ with our parameters $2 \kappa,-\xi, z$ (note though that there is a small typo in $[6,(4.4)])$. The dynamical crossing symmetry (2.23) of $R_{B a}(z, \xi)$ corresponds to the crossing symmetry [6, Sect. 4.1 and (2.11)] of the Boltzmann weights $W_{8 v}$ Sos . 
We write $B_{8 v \operatorname{Sos}}\left(\begin{array}{cc}b & c \\ & a\end{array} z, \xi\right)$ for the boundary Boltzmann weights associated to the dynamical $K$-matrix $K_{B a}(z, \xi)$ (see (2.27)). The nonzero values are

$$
\begin{aligned}
& B_{8 v \operatorname{SOS}}\left(\begin{array}{l}
a \pm 1 \\
a \pm 1
\end{array} \mid z, \xi\right)=K_{B a, \pm}^{ \pm}\left(z, \frac{\xi}{2}-\kappa a\right)=\alpha_{B a}\left(z, \pm \frac{\xi}{2} \mp \kappa a\right), \\
& B_{8 v \operatorname{SOS}}\left(\begin{array}{c}
a \neq 1 \\
a \pm 1
\end{array} \mid z, \xi\right)=K_{B a, \mp}^{ \pm}\left(z, \frac{\xi}{2}-\kappa a\right)=\beta_{B a}\left(z, \pm \frac{\xi}{2} \mp \kappa a\right),
\end{aligned}
$$

where $\alpha_{B a}$ and $\beta_{B a}$ are explicitly given by (2.22).

Corollary 2.23. B 8 vSOS is a 4-parameter family of solutions of the boundary YangBaxter equations (2.28) with respect to the Boltzmann weights $W_{8 v}$ Sos.

Proof. By Proposition 2.16 the matrix $K_{B a}(z, \xi)$ is also a right dynamical $K$-matrix with respect to the gauged dynamical $R$-matrix $R_{8 v S O S}(z, \xi)$. Now use Proposition 2.22.

Solutions to the boundary Yang-Baxter equations for the eight vertex face model, or equivalently, by vertex-face correspondences, to reflection equations for the eight vertex model itself, have been computed by direct means in $[32,33,67]$ from the vertex perspective and in $[6,18,43]$ from the face perspective (see [31] for a discussion of the vertex-face correspondence in this context). See [6,4.3] for a direct derivation of the solutions of the boundary Yang-Baxter equations for the eight vertex face model, which are also parametrised (but in a different way) by four degrees of freedoms.

2.8. Boundary $q K Z B$ equations. A unitary solution of the dynamical quantum YangBaxter equation satisfying the ice-rule gives rise to cocycles of the extended affine symmetric group [19]. This leads to a compatible system of $q$-difference equations, known as the quantum Knizhnik-Zamolodchikov-Bernard (KZB) equations, see [19, 21]. In this subsection we establish the analog of this result in the presence of boundaries, resulting in a cocycle of the affine Weyl group of type $C_{n}$ and a new type of compatible systems of $q$-difference equations, which we will call boundary quantum KZB equations.

We return to the setting of Sect. 2.5. If $f: \mathbb{C} \rightarrow V^{\otimes n}$ is a meromorphic $V^{\otimes n}$-valued function then we define

$$
\left(T_{\alpha h_{i}} f\right)(\xi):=\sum_{\underline{\mu}} f_{\underline{\mu}}\left(\xi+\alpha \mu_{i}\right)
$$

if $f(\xi)=\sum_{\underline{\mu}} f_{\underline{\mu}}(\xi)$ is the decomposition of $f$ as the sum of $\left(V^{\otimes n}\right)_{\underline{\mu}}$-valued meromorphic functions $f_{\mu}$. In case of a meromorphic $V^{\otimes n}$-valued function $f(\mathbf{z}, \xi)$, the parameter-shift of $T_{\alpha h_{i}}$ is in the dynamical parameter $\xi$. When $n=1$ then we write $T_{\alpha h}$ for $T_{\alpha h_{1}}$.

Let $S(\mathbf{z}, \xi)$ be a family of linear operators on $V^{\otimes n}$ depending meromorphically on $(\mathbf{z}, \xi) \in \mathbb{C}^{n} \times \mathbb{C}$. Consider the associated matrix difference operator $T_{-\kappa h_{i}} S T_{\kappa h_{i}}$, acting complex linearly on the space $\mathcal{M}\left(\mathbb{C}^{n} \times \mathbb{C}\right) \otimes V^{\otimes n}$ of meromorphic $V^{\otimes n}$-valued functions on $\mathbb{C}^{n} \times \mathbb{C}$. It is a matrix valued difference operator in the dynamical parameter $\xi$, depending meromorphically on $\mathbf{z} \in \mathbb{C}^{n}$. We therefore also denote it by $T_{-\kappa h_{i}} S(\mathbf{z}, \cdot) T_{-\kappa h_{i}}$. 
Let $\underline{K}(z, \xi)$ be a linear operators on $V$ depending meromorphically on $(z, \xi) \in \mathbb{C}^{2}$. Let $P: V \otimes V \rightarrow V \otimes V$ be the permutation operator and consider the $\operatorname{End}_{\mathbb{C}}\left(V^{\otimes n}\right)$ valued difference operator in $w$ defined by

$$
\begin{aligned}
M^{S_{0}}(\mathbf{z}) & :=\left(T_{-\kappa h} \underline{K}\left(\frac{1}{2}-z_{1}, \cdot\right) T_{\kappa h}\right)_{1} \\
& =T_{-\kappa h_{1}} \underline{K}_{1}\left(\frac{1}{2}-z_{1}, \cdot\right) T_{\kappa h_{1}} .
\end{aligned}
$$

Remark 2.24. Let $\pi_{\mu}: V \rightarrow V_{\mu}$ be the projection along the decomposition $V=\bigoplus_{v} V_{\nu}$. For $f(\xi) \in V$ depending meromorphically on $\xi$ we have

$$
\left(\left(T_{-\kappa h} \underline{K}(z, \cdot) T_{\kappa h}\right) f\right)(\xi)=\sum_{\mu, v} \underline{K}_{v}^{\mu}(z, \xi-\kappa v) f_{\mu}(\xi+\kappa(\mu-v))
$$

where $f_{\mu}(\xi):=\pi_{\mu}(f(\xi))$ and $\underline{K}_{\nu}^{\mu}(z, \xi):=\left.\pi_{\nu} \underline{K}(z, \xi)\right|_{V_{\mu}}$. Hence if the left dynamical $K$-matrix $\underline{K}(z, \xi)$ satisfies

$$
[\underline{K}(z, \xi), h]=0
$$

then $M^{s_{0}}(\mathbf{z})=\underline{K}_{1}\left(\frac{1}{2}-z_{1}, \cdot-\kappa h_{1}\right)$ (which thus simply acts on the space of meromorphic $V^{\otimes n}$-valued functions in $(\mathbf{z}, \xi) \in \mathbb{C}^{n} \times \mathbb{C}$ as the linear operator $\left.\underline{K}_{1}\left(\frac{1}{2}-z_{1}, \xi-\kappa h_{1}\right)\right)$.

We have now the following affine version of Proposition 2.14.

Proposition 2.25. Let $R(z, \xi)$ be a linear operator on $V \otimes V$ and $K(z, \xi), \underline{K}(z, \xi)$ linear operators on $V$, all depending meromorphically on $(z, \xi) \in \mathbb{C}^{2}$. Suppose that $R(z, \xi)$ satisfies the ice-rule (2.12). The following two statements are equivalent.

(1) The operators (2.30) and (2.19) are part of a (necessarily unique) set $\left\{M^{v}(\mathbf{z})\right\}_{v \in W}$ of $\operatorname{End}_{\mathbb{C}}\left(V^{\otimes n}\right)$-valued meromorphic difference operators $M^{v}(\mathbf{z})$ in $\xi$, depending meromorphically on $\mathbf{z} \in \mathbb{C}^{n}$, and satisfying the cocycle conditions

$$
M^{e}(\mathbf{z})=\mathrm{Id}, \quad M^{u v}(\mathbf{z})=M^{u}(\mathbf{z}) M^{v}\left(u^{-1} \mathbf{z}\right)
$$

for all $u, v \in W$

(2) $R(z, \xi)$ is a unitary dynamical $R$-matrix, and $\underline{K}(z, \xi)$ and $K(z, \xi)$ are associated unitary left and right dynamical $K$-matrices respectively.

Proof. For $v \in W_{0}$ the $M^{v}(\mathbf{z})$ coincide with the cocycle values from Proposition 2.14. The extension to the affine setup now follows by a direct computation.

Proposition 2.25 should be compared to [19, Proposition 4.1 and Theorem 4.2] (see also [21]), which deals with the extended affine symmetric group.

Remark 2.26. Note that the cocycle values $M^{v}(\mathbf{z})(v \in W)$ are $\operatorname{End}_{\mathbb{C}}\left(V^{\otimes n}\right)$-valued difference operators in $\xi$, depending meromorphically on $\mathbf{z} \in \mathbb{C}^{n}$. For $v \in W_{0}$ they simply act as multiplication operators,

$$
\left(M^{v}(\mathbf{z}) f(\mathbf{z}, \cdot)\right)(\xi)=M^{v}(\mathbf{z}, \xi) f(\mathbf{z}, \xi), \quad v \in W_{0},
$$

with the linear operator $M^{v}(\mathbf{z}, \xi)$ on $V^{\otimes n}$ as defined in Proposition 2.14. In fact, if $\underline{K}(\mathbf{z}, \xi)$ satisfies $(2.31)$ then all cocycle values $M^{v}(\mathbf{z})(v \in W)$ act as multiplication operators in view of Remark 2.24. 
Definition 2.27. Suppose that $R(z, \xi): V \otimes V \rightarrow V \otimes V$ is a unitary solution of the dynamical quantum Yang-Baxter equation satisfying the ice-rule and suppose that $\underline{K}(z, \xi)$ and $K(z, \xi)$ are associated unitary left and right dynamical $K$-matrices. Let $\left\{M^{u}(\mathbf{z})\right\}_{u \in W}$ be the $W$-cocycle of difference operators in $\xi$ as defined in Proposition 2.25 .

A $V^{\otimes n}$-valued meromorphic function $f(\mathbf{z}, \xi)$ in $(\mathbf{z}, \xi) \in \mathbb{C}^{n} \times \mathbb{C}$ is said to satisfy the boundary quantum Knizhnik-Zamolodchikov-Bernard (KZB) equations if

$$
M^{\tau(\lambda)}(\mathbf{z}) f(\mathbf{z}-\lambda, \cdot)=f(\mathbf{z}, \cdot) \quad \forall \lambda \in \mathbb{Z}^{n} .
$$

The boundary quantum KZB equations (2.32) form a compatible systems of difference equations in both $\mathbf{z}$ and $\xi$. Note that the translation action on the dynamical parameter $\xi$ is hidden in the transport operators $M^{\tau(\lambda)}(\mathbf{z})\left(\lambda \in \mathbb{Z}^{n}\right)$.

Remark 2.28. The space of $V^{\otimes n}$-valued meromorphic functions $f(\mathbf{z}, \xi)$ in $(\mathbf{z}, \xi) \in \mathbb{C}^{n} \times$ $\mathbb{C}$ satisfying the boundary quantum KZB equations (2.32) is invariant for the $W_{0}$-action

$$
(v \cdot f)(\mathbf{z}, \xi):=M^{v}(\mathbf{z}, \xi) f\left(v^{-1} \mathbf{z}, \xi\right), \quad v \in W_{0} .
$$

Remark 2.29. If the left dynamical $K$-matrix $\underline{K}(z, \xi)$ satisfies (2.31), then the transport operators of the boundary quantum KZB equations are linear operators $M^{\tau(\lambda)}(\mathbf{z}, \xi)$ on $V^{\otimes n}$ depending meromorphically on $(\mathbf{z}, \xi)$ (see Remark 2.26). Hence, under the additional assumption (2.31) on $\underline{K}(z, \xi)$, the boundary quantum KZB equations reduce to the compatible system

$$
M^{\tau(\lambda)}(\mathbf{z}, \xi) f(\mathbf{z}-\lambda, \xi)=f(\mathbf{z}, \xi) \quad \forall \lambda \in \mathbb{Z}^{n}
$$

of difference equations acting only on the spectral parameters $\mathbf{z}$.

The boundary quantum KZB equations are equivalent to the set of equations

$$
M^{\tau\left(-e_{i}\right)}(\mathbf{z}) f\left(\mathbf{z}+e_{i}, \cdot\right)=f(\mathbf{z}, \cdot) \quad i=1, \ldots, n .
$$

The left hand side of (2.33) can be explicitly expressed in terms of the dynamical $R$-and $K$-matrices using the cocycle property of $\left\{M^{v}(\mathbf{z})\right\}_{v \in W}$ (Proposition 2.25) and (2.1). We give the explicit expression of (2.33) for $i=1$, the explicit expressions for arbitrary $i$ then follow from

$$
M^{\tau\left(-e_{i+1}\right)}(\mathbf{z})=M^{s_{i}}(\mathbf{z}) M^{\tau\left(-e_{i}\right)}\left(s_{i} \mathbf{z}\right) M^{s_{i}}\left(s_{i} \mathbf{z}+e_{i}\right)
$$

recursively. For $i=1$ the left hand side of (2.33) is

$$
\begin{aligned}
& \left(M^{\tau\left(-e_{1}\right)}(\mathbf{z}) f\left(\mathbf{z}+e_{1}, \cdot\right)\right)(\xi)=R_{21}\left(z_{1}-z_{2}, 2 \xi\right) R_{31}\left(z_{1}-z_{3}, 2 \xi-2 \kappa h_{2}\right) \\
& \quad \times \cdots \times R_{n 1}\left(z_{1}-z_{n}, 2 \xi-2 \kappa\left(h_{2}+\cdots+h_{n-1}\right)\right) K_{1}\left(z_{1}, \xi-\kappa\left(h_{2}+h_{3}+\cdots+h_{n}\right)\right) \\
& \quad \times R_{1 n}\left(z_{1}+z_{n}, 2 \xi-2 \kappa\left(h_{2}+h_{3}+\cdots+h_{n-1}\right)\right) \cdots R_{13}\left(z_{1}+z_{3}, 2 \xi-2 \kappa h_{2}\right) \\
& \quad \times R_{12}\left(z_{1}+z_{2}, 2 \xi\right) \sum_{\underline{\mu}, \underline{\underline{v}}} \underline{K}_{v_{1}}^{\mu_{1}}\left(\frac{1}{2}+z_{1}, \xi-\kappa v_{1}\right) f_{\mu}\left(\mathbf{z}+e_{1}, \xi+\kappa\left(\mu_{1}-v_{1}\right)\right) .
\end{aligned}
$$


In the special case that the left dynamical $K$-matrix $K(z, \xi)$ satisfies (2.31), the left hand side of (2.33) for $i=1$ takes on the simpler form

$$
\begin{aligned}
& M^{\tau\left(-e_{1}\right)}(\mathbf{z}, \xi) f\left(\mathbf{z}+e_{1}, \xi\right)=R_{21}\left(z_{1}-z_{2}, 2 \xi\right) R_{31}\left(z_{1}-z_{3}, 2 \xi-2 \kappa h_{2}\right) \\
& \quad \times \cdots \times R_{n 1}\left(z_{1}-z_{n}, 2 \xi-2 \kappa\left(h_{2}+\cdots+h_{n-1}\right)\right) K_{1}\left(z_{1}, \xi-\kappa\left(h_{2}+h_{3}+\cdots+h_{n}\right)\right) \\
& \quad \times R_{1 n}\left(z_{1}+z_{n}, 2 \xi-2 \kappa\left(h_{2}+h_{3}+\cdots+h_{n-1}\right)\right) \cdots R_{13}\left(z_{1}+z_{3}, 2 \xi-2 \kappa h_{2}\right) \\
& \quad \times R_{12}\left(z_{1}+z_{2}, 2 \xi\right) \underline{K}_{1}\left(\frac{1}{2}+z_{1}, \xi-\kappa h_{1}\right) f\left(\mathbf{z}+e_{1}, \xi\right)
\end{aligned}
$$

due to Remarks 2.24 and 2.29.

We now apply the construction of the boundary quantum KZB equations to Baxter's dynamical $R$-matrix $R_{B a}(z, \xi)$ and the associated 4-parameter family of unitary dynamical $K$-matrices. So in the following corollary we take $V=\mathbb{C}^{2}=\mathbb{C} v_{+} \oplus \mathbb{C} v_{-}$ and $h v_{\epsilon}=\epsilon v_{\epsilon}$ for $\epsilon \in\{ \pm\}$.

Corollary 2.30. Let $R_{B a}(z, \xi)$ be Baxter's dynamical $R$-matrix given explicitly by (2.20) (it depends on $q$ and the bulk coupling parameter $\kappa$ ). Fix four left boundary coupling parameters $\zeta_{l}, \zeta_{l}^{\prime}, v_{l}, v_{l}^{\prime}$ and four right boundary coupling parameters $\zeta, \zeta^{\prime}, v, v^{\prime}$ and set

$$
\begin{aligned}
& \underline{K}_{B a}^{l}(z, \xi):=K_{B a}\left(z,-\xi ; \zeta_{l}, \zeta_{l}^{\prime}, v_{l}, v_{l}^{\prime}\right), \\
& K_{B a}^{r}(z, \xi):=K_{B a}\left(z, \xi ; \zeta, \zeta^{\prime}, v, v^{\prime}\right)
\end{aligned}
$$

with $K_{B a}\left(z, \xi ; \zeta, \zeta^{\prime}, v, v^{\prime}\right)$ given by (2.21). Then $\underline{K}_{B a}^{l}(z, \xi)$ and $K_{B a}^{r}(z, \xi)$ are unitary left and right dynamical $K$-matrices associated to $R_{B a}(z, \xi)$ respectively. Let $\left\{M_{B a}^{v}(\mathbf{z})\right\}_{v \in W}$ be the associated $W$-cocycle of $\operatorname{End}_{\mathbb{C}}\left(\left(\mathbb{C}^{2}\right)^{\otimes n}\right)$-valued difference operators in $\xi$ depending meromorphically on $\mathbf{z} \in \mathbb{C}^{n}$ (see Proposition 2.25). The corresponding quantum $K Z B$ equations

$$
M_{B a}^{\tau(\lambda)}(\mathbf{z}) f(\mathbf{z}-\lambda, \cdot)=f(\mathbf{z}, \cdot), \quad \forall \lambda \in \mathbb{Z}^{n}
$$

for meromorphic $\left(\mathbb{C}^{2}\right)^{\otimes n}$-valued functions $f(\mathbf{z}, \xi)$ in $(\mathbf{z}, \xi) \in \mathbb{C}^{n} \times \mathbb{C}$ is a compatible system of difference equations in $\mathbf{z}$ and $\xi$ with elliptic coefficients that depends, besides on $q$, on nine coupling parameters $\kappa, \zeta_{l}, \zeta_{l}^{\prime}, v_{l}, v_{l}^{\prime}, \zeta, \zeta^{\prime}, v, v^{\prime}$.

Proof. The fact that $K^{l}(z, \xi)$ and $K^{r}(z, \xi)$ are left and right dynamical $K$-matrices respectively follows from Corollary 2.18 and Remark 2.13 .

Remark 2.31. It is natural to expect that a vertex-face transformation (cf. [31]) transforms the nine parameter family (2.34) of elliptic boundary quantum KZB equations into a nine parameter family of elliptic boundary qKZ equations defined in terms of the XYZ spin- $\frac{1}{2}$ $R$-matrix and its associated $K$-matrices. We expect that a difference Cherednik-Matsuo type of correspondence will relate the solutions of these compatible systems of difference equations to common eigenfunctions of the nine parameter family of elliptic Ruijsenaars' systems of type C introduced by van Diejen [14] and Komori and Hikami [42]. 


\section{The Connection Problem for Quantum Affine KZ Equations}

Cherednik [11, Sect. 1.3.2] introduced the Baxterization of a finite dimensional affine Hecke algebra module using the affine intertwiners of the associated double affine Hecke algebra. It leads to a consistent system of first-order difference equations acting on vector-valued multivariate meromorphic functions, called the quantum affine KnizhnikZamolodchikov (KZ) equations. These equations are equivariant with respect to a natural action of the underlying Weyl group.

A natural basis of power series solutions of the quantum affine KZ equations, defined in terms of their asymptotic behaviour deep in a fixed Weyl chamber, was constructed in $[50,51,63]$ in case of minimal principal series. We extend this result to arbitrary principal series modules, and we also solve the associated connection problem. the affine Hecke algebra of type $C$.

We explain how a special case of this general theory leads to the results on lattice models with boundaries as discussed in the previous section.

3.1. Notations. We recall here some of the notations from [64, Sects. 1.1 and 2], which are well suited to treat the twisted and untwisted Cherednik-Macdonald theory at the same time (see $[64,66]$ for details). The initial data is given by a five tuple $D=\left(R_{0}, \Delta_{0}, \bullet, \Lambda, \widetilde{\Lambda}\right)$, which is defined as follows.

The first entry $R_{0}$ is a finite, reduced crystallographic root system in an Euclidean space $(E,(\cdot, \cdot))$, irreducible within the subspace $V$ of $E$ spanned by the roots. The second entry $\Delta_{0}=\left(\alpha_{1}, \ldots, \alpha_{n}\right)$ is an ordered basis of the root system $R_{0}$. The third entry $\bullet$ equals $u$ or $t$ (" $u$ " stands for untwisted and " $t$ " for twisted). The fourth and fifth entries $\Lambda$ and $\widetilde{\Lambda}$ are lattices that are defined as follows.

Write $R_{0}=R_{0}^{+} \cup R_{0}^{-}$for the decomposition of $R_{0}$ in positive and negative roots with respect to the basis $\Delta_{0}$. The root system $\widetilde{R}_{0} \bullet$-dual to $R_{0}$ is defined as

$$
\widetilde{R}_{0}:=\left\{\widetilde{\alpha}:=\mu_{\alpha} \alpha^{\vee} \mid \alpha \in R_{0}\right\},
$$

where $\alpha^{\vee}=\frac{2 \alpha}{|\alpha|^{2}}$ is the co-root of $\alpha$ and

$$
\mu_{\alpha}:= \begin{cases}1 & \text { if } \bullet=u, \\ \frac{|\alpha|^{2}}{2} & \text { if } \bullet=t .\end{cases}
$$

So in the untwisted case $(\bullet=u), \widetilde{R}_{0}$ is the co-root system $R_{0}^{\vee}=\left\{\alpha^{\vee}\right\}_{\alpha \in R_{0}}$, while in the twisted case $(\bullet=t), \widetilde{R}_{0}=R_{0}$. Let $W_{0} \subseteq O(E)$ be the Weyl group of $R_{0}$, generated by the orthogonal reflections $s_{\alpha}$ in the hyperplanes $\alpha^{-1}(0)\left(\alpha \in R_{0}\right)$. It is a Coxeter group with Coxeter generators the simple reflections $s_{i}:=s_{\alpha_{i}}=s_{\widetilde{\alpha}_{i}}(i=1, \ldots, n)$.

Write $Q$ and $\widetilde{Q}$ for the root lattices of $R_{0}$ and $\widetilde{R}_{0}$ respectively. Let $Q^{\vee}$ and $\widetilde{Q}^{\vee}$ be the co-root lattice of $R_{0}$ and $\widetilde{R}_{0}$ respectively. The fourth entry $\Lambda \subseteq E$ in the five-tuple $D$ of initial data is a full lattice in $E$ satisfying the conditions

$$
Q \subseteq \Lambda, \quad\left(\Lambda, Q^{\vee}\right) \subseteq \mathbb{Z}
$$

The fifth entry $\widetilde{\Lambda} \subseteq E$ is a full lattice in $E$ satisfying the conditions

$$
\widetilde{Q} \subseteq \widetilde{\Lambda}, \quad\left(\widetilde{\Lambda}, \widetilde{Q}^{\vee}\right) \subseteq \mathbb{Z}
$$


We associate to $D$ the reduced irreducible affine root system

$$
R^{\bullet}:=\left\{\alpha^{(r)}:=\mu_{\alpha} r c+\alpha\right\}_{r \in \mathbb{Z}, \alpha \in R_{0}},
$$

where $\mu_{\alpha} r c+\alpha$ is the affine linear function $v \mapsto \mu_{\alpha} r+(\alpha, v)$ on $E$. Let $s_{\alpha}(r)$ be the orthogonal reflection in the affine hyperplane $\left(\alpha^{(r)}\right)^{-1}(0) \subset E$. Then $s_{\alpha^{(r)}}=\tau(-r \widetilde{\alpha}) s_{\alpha}$ with $\tau\left(v^{\prime}\right): V \rightarrow V$ for $v^{\prime} \in V$ the translation operator $v \mapsto v+v^{\prime}$. In particular, the affine Weyl group $W^{\bullet}$ associated to $R^{\bullet}$, which is defined to be the group generated by the $s_{\alpha^{(r)}}\left(\alpha \in R_{0}, r \in \mathbb{Z}\right)$, is isomorphic to $W_{0} \ltimes \widetilde{Q}$. We extend $R^{\bullet}$ to a (possibly nonreduced) irreducible affine root system $R(D)$ by adding to $R^{\bullet}$ the affine roots $2 \alpha^{(r)}$ for $r \in \mathbb{Z}$ and $\alpha \in R_{0}$ satisfying $\left(\Lambda, \alpha^{\vee}\right) \subseteq 2 \mathbb{Z}$.

The basis $\Delta_{0}$ of $R_{0}$ extends to a basis $\Delta:=\left(\alpha_{0}, \alpha_{1}, \ldots, \alpha_{n}\right)$ of $R^{\bullet}$ and $R(D)$ with the additional simple affine root $\alpha_{0}=\mu_{\psi} c-\psi$ with $\psi \in R_{0}$ the highest (reps. highest short) root with respect to $\Delta_{0}$ if $\bullet=u($ resp. $\bullet=t)$. The basis $\Delta$ of $R^{\bullet}$ gives a decomposition $R^{\bullet}=R^{\bullet,+} \cup R^{\bullet,-}$ of $R^{\bullet}$ in positive and negative affine roots. We write $s_{0}:=s_{\alpha^{(0)}}$. Note that $s_{0}=\tau(\widetilde{\psi}) s_{\psi}$. The affine Weyl group is a Coxeter group with Coxeter generators $s_{0}, s_{1}, \ldots, s_{n}$. The corresponding braid relations are of the form

$$
s_{i} s_{j} \cdots=s_{j} s_{i} \cdots \quad\left(m_{i j} \text { factors on both sides }\right)
$$

for $0 \leq i \neq j \leq n$, where the $m_{i j} \in\{2,3,4,6, \infty\}$ can be read off from the associated Coxeter graph in the usual manner.

The extended affine Weyl group is defined to be $W:=W_{0} \ltimes \widetilde{\Lambda}$. It acts on $E$ by reflections and $\widetilde{\Lambda}$-translations. Its contragredient action on the space of affine linear functions on $E$ preserves $R^{\bullet}$ and $R(D)$. The length of $w \in W$ is defined to be

$$
l(w):=\#\left(R^{\bullet,+} \cap w^{-1} R^{\bullet,-}\right) .
$$

Then $\Omega:=\{w \in W \mid l(w)=0\}$ is an abelian subgroup of $W$, isomorphic to $\widetilde{\Lambda} / \widetilde{Q}$. Conjugation by $\Omega$ stabilises $W^{\bullet}$ and

$$
W \simeq \Omega \ltimes W^{\bullet}
$$

More precisely $\Omega$, in its action on affine linear functions on $E$, is preserving the set $\left\{\alpha_{0}, \alpha_{2}, \ldots, \alpha_{n}\right\}$ of simple affine roots. For $\varpi \in \Omega$ the induced bijection on the index set $\{0, \ldots, n\}$ will also be denoted by $\varpi$, so $\varpi\left(\alpha_{j}\right)=\alpha_{\varpi(j)}$ for $0 \leq j \leq n$ and $\varpi \in \Omega$. Hence conjugation by $\Omega$ already stabilises the set $\left\{s_{0}, \ldots, s_{n}\right\}$ of simple reflections,

$$
\varpi s_{j} \varpi^{-1}=s_{\varpi(j)}, \quad \varpi \in \Omega, j \in\{0, \ldots, n\} .
$$

Write

$$
\widetilde{\Lambda}_{\min }^{+}:=\left\{v \in \widetilde{\Lambda} \mid\left(v, \widetilde{\alpha}^{\vee}\right) \in\{0,1\} \quad \forall \alpha \in R_{0}^{+}\right\} .
$$

For $v \in \widetilde{\Lambda}_{\min }^{+}$let $u(v) \in W$ be the element of minimal length in $\tau(v) W_{0}$. Define

$$
v(v):=u(v)^{-1} \tau(v) \in W_{0},
$$

so that $\tau(v)=u(v) v(v)$ and $l(\tau(v))=l(u(v))+l(v(v))$. Then it is known that

$$
\Omega=\{u(v)\}_{v \in \tilde{\Lambda}_{\text {min }}^{+}} .
$$


There is an involution on the collection of all initial data $D=\left(R_{0}, \Delta_{0}, \bullet, \Lambda, \widetilde{\Lambda}\right)$, mapping $D$ to

$$
\widetilde{D}:=\left(\widetilde{R}_{0}, \widetilde{\Delta}_{0}, \bullet, \widetilde{\Lambda}, \Lambda\right)
$$

where $\widetilde{\Delta}_{0}:=\left(\widetilde{\alpha}_{1}, \ldots, \widetilde{\alpha}_{n}\right)$. The (extended) affine Weyl group, affine root systems etc., will be denoted by adding tildes. Note that for both the twisted and the untwisted case, the affine simple root associated to $\widetilde{R}^{\bullet}$ is given by $\widetilde{\alpha}_{0}=\mu_{\widetilde{\theta}} c-\widetilde{\theta}$ with $\theta \in R_{0}^{+}$the highest short root (see [64, Sect. 2.2]). In particular, $\widetilde{s}_{0}=\tau(\theta) s_{\theta}$.

Finally we introduce multiplicity functions. A multiplicity function is a $W$-invariant function $\kappa: R(D) \rightarrow \mathbb{R}$. We write $\kappa_{a} \in \mathbb{R}$ for the value of $\kappa$ at $a \in R(D)$ and $\kappa_{j}:=\kappa_{\alpha_{j}}$ for $0 \leq j \leq n$. We furthermore write $\kappa_{2 \alpha^{(r)}}:=\kappa_{\alpha^{(r)}}$ if $2 \alpha^{(r)} \notin R(D)$. For a finite root $\alpha \in R_{0}$ we define the Askey-Wilson parameters associated to $\kappa$ and $\alpha$ by

$$
\left\{a_{\alpha}, b_{\alpha}, c_{\alpha}, d_{\alpha}\right\}:=\left\{q^{\kappa_{\alpha}+\kappa_{2 \alpha}},-q^{\kappa_{\alpha}-\kappa_{2 \alpha}}, q_{\alpha} q^{\kappa_{\alpha}(1)+\kappa_{2 \alpha}(1)},-q_{\alpha} q^{\kappa_{\alpha}(1)-\kappa_{2 \alpha}(1)}\right\},
$$

where $q_{\alpha}:=q^{\mu_{\alpha}}$. We furthermore write $\kappa_{j}:=\kappa_{\alpha_{j}}$ for $0 \leq j \leq n$.

Given a multiplicity function $\kappa$ on $R(D)$, there exists a unique multiplicity function $\widetilde{\kappa}$ on $R(\widetilde{D})$ satisfying

$$
\widetilde{\kappa}_{\widetilde{\alpha}}=\kappa_{\alpha}, \quad \widetilde{\kappa}_{\widetilde{\alpha}^{(1)}}=\kappa_{2 \alpha}, \quad \widetilde{\kappa}_{2 \widetilde{\alpha}}=\kappa_{\alpha^{(1)}}, \quad \widetilde{\kappa}_{2 \widetilde{\alpha}^{(1)}}=\kappa_{2 \alpha^{(1)}}
$$

for all $\alpha \in R_{\widetilde{D}}$. We write the Askey-Wilson parameters associated to $\widetilde{\kappa}$ and $\widetilde{\alpha} \in \widetilde{R}_{0}$ by $\left\{\widetilde{a}_{\alpha}, \widetilde{b}_{\alpha}, \widetilde{c}_{\alpha}, \widetilde{d}_{\alpha}\right\}$ (we abuse notation here by writing $\alpha$ as sublabel instead of $\widetilde{\alpha}$ ). In terms of the original multiplicity function $\kappa$ they are explicitly given by

$$
\left\{\widetilde{a}_{\alpha}, \widetilde{b}_{\alpha}, \widetilde{c}_{\alpha}, \widetilde{d}_{\alpha}\right\}=\left\{q^{\kappa_{\alpha}+\kappa_{\alpha}(1)},-q^{\kappa_{\alpha}-\kappa_{\alpha}(1)}, q_{\alpha} q^{\kappa_{2 \alpha}+\kappa_{2 \alpha}(1)},-q_{\alpha} q^{\kappa_{2 \alpha}-\kappa_{2 \alpha}(1)}\right\},
$$

+ hence they are obtained from the Askey-Wilson parameters $\left\{a_{\alpha}, b_{\alpha}, c_{\alpha}, d_{\alpha}\right\}$ by interchanging the values $\kappa_{2 \alpha}$ and $\kappa_{\alpha^{(1)}}$. We also write $\widetilde{\kappa}_{j}:=\widetilde{\kappa}_{\widetilde{\alpha}_{j}}$ for $0 \leq j \leq n$.

\subsection{Principal series representations.}

Definition 3.1. The affine Hecke algebra $H^{\bullet}(\kappa)$ is the unital associative algebra over $\mathbb{C}$ generated by $T_{j}(0 \leq j \leq n)$ with defining relations

$$
T_{i} T_{j} T_{i} \cdots=T_{j} T_{i} T_{j} \cdots \quad\left(m_{i j} \text { factors on both sides }\right)
$$

for $0 \leq i \neq j \leq n$ and $\left(T_{j}-q^{-\kappa_{j}}\right)\left(T_{j}+q^{\kappa_{j}}\right)=0$ for $0 \leq j \leq n$.

We write $H_{0}(\kappa)$ for the unital subalgebra of $H^{\bullet}(\kappa)$ generated by $T_{i}(1 \leq i \leq n)$.

The abelian group $\Omega$ of length zero elements in $W$ acts by algebra automorphisms on $H^{\bullet}(\kappa)$, with $\varpi \in \Omega$ acting by $T_{j} \mapsto T_{\varpi(j)}$ for $0 \leq j \leq n$. We write

$$
H(\kappa):=\Omega \ltimes H^{\bullet}(\kappa)
$$

for the corresponding crossed product algebra. We call it the extended affine Hecke algebra associated to $D$ and $\kappa$. The additional generators in $H(\kappa)$ parametrized by $\Omega$ are denoted by $T_{\varpi}(\varpi \in \Omega)$. For $w \in W$ we write

$$
T_{w}:=T_{\varpi} T_{j_{1}} T_{j_{2}} \cdots T_{j_{r}} \in H(\kappa)
$$

if $w=\varpi s_{j_{1}} s_{j_{2}} \cdots s_{j_{r}}$ in $W$ with $\varpi \in \Omega, 0 \leq j \leq n$ and $l(w)=r$. It gives a well defined complex linear basis $\left\{T_{w}\right\}_{w \in W}$ of $H(\kappa)$. 
Write

$$
\widetilde{\Lambda}^{+}:=\left\{v \in \widetilde{\Lambda} \mid\left(v, \widetilde{\alpha}^{\vee}\right) \geq 0 \quad \forall \alpha \in R_{0}^{+}\right\}
$$

and define for $v \in \widetilde{\Lambda}$,

$$
Y^{\nu}:=T_{\tau(\lambda)} T_{\tau(\mu)}^{-1} \in H(\kappa)
$$

if $v=\lambda-\mu$ with $\lambda, \mu \in \widetilde{\Lambda}^{+}$. The $Y^{v}$ are well defined and satisfy

$$
Y^{v} Y^{v^{\prime}}=Y^{v+v^{\prime}}, \quad Y^{0}=1
$$

for $v, v^{\prime} \in \widetilde{\Lambda}$. The extended affine Hecke algebra $H(\kappa)$ is generated by $H_{0}(\kappa)$ and the commuting elements $Y^{v}(v \in \widetilde{\Lambda})$. The additional relations characterizing $H(\kappa)$ in terms of the generators $T_{i}(1 \leq i \leq n)$ and $Y^{v}(\nu \in \widetilde{\Lambda})$ are the Bernstein-Zelevinsky-Lusztig relations

$$
Y^{v} T_{i}=T_{i} Y^{s_{i} v}+\left(q^{\widetilde{\kappa}_{i}} \frac{\left(1-q^{-\widetilde{\kappa}_{i}-\widetilde{\kappa}_{2} \widetilde{\alpha}_{i}} Y^{-\widetilde{\alpha}_{i}}\right)\left(1+q^{-\widetilde{\kappa}_{i}+\widetilde{\kappa}_{2} \widetilde{\alpha}_{i} Y^{-\widetilde{\alpha}_{i}}}\right)}{1-Y^{-2 \widetilde{\alpha}_{i}}}-q^{-\widetilde{\kappa}_{i}}\right)\left(Y^{s_{i} v}-Y^{v}\right)
$$

for $1 \leq i \leq n$ and $v \in \widetilde{\Lambda}($ see $[66,(3.4)])$.

Let $E_{\mathbb{C}}:=\mathbb{C} \otimes E$ be the complexification of $E$ and extend the scalar product $(\cdot, \cdot)$ to a complex bilinear form on $E_{\mathbb{C}}$. For $I \subseteq\{1, \ldots, n\}$ write $H_{I}(\kappa)$ for the subalgebra of $H_{I}(\kappa)$ generated by $T_{i}(i \in I)$ and $Y^{v}(v \in \widetilde{\Lambda})$. Set

$$
E_{\mathbb{C}, I}^{\kappa}:=\left\{\gamma \in E_{\mathbb{C}} \mid\left(\widetilde{\alpha}_{i}, \gamma\right)=\widetilde{\kappa}_{\widetilde{\alpha}_{i}}+\widetilde{\kappa}_{2} \widetilde{\alpha}_{i} \quad \forall i \in I\right\}
$$

Lemma 3.2. Let $I \subseteq\{1, \ldots, n\}$ and $\gamma \in E_{\mathbb{C}, I}^{\kappa}$. There exists a unique unital algebra map

$$
\chi_{I, \gamma}: H_{I}(\kappa) \rightarrow \mathbb{C}
$$

satisfying

$$
\begin{gathered}
\chi_{I, \gamma}\left(T_{i}\right)=q^{-\kappa_{i}}, \quad \forall i \in I, \\
\chi_{I, \gamma}\left(Y^{v}\right)=q^{-(v, \gamma)}, \quad \forall v \in \widetilde{\Lambda} .
\end{gathered}
$$

Proof. The assumption $\gamma \in E_{\mathbb{C}, I}^{\kappa}$ ensures that the assignment (3.2) respects the crossing relations (3.1) (we use here that $\widetilde{\kappa}_{i}=\kappa_{i}$ for $i=1, \ldots, n$ ).

Definition 3.3. Let $I \subseteq\{1, \ldots, n\}$ and $\gamma \in E_{\mathbb{C}, I}^{\kappa}$. The induced representation

$$
M_{I}(\gamma):=\operatorname{Ind}_{H_{I}(\kappa)}^{H(\kappa)}\left(\mathbb{C}_{\chi_{I, \gamma}}\right)
$$

is called the principal series representation associated to $I$, with central character $q^{-\gamma}$. We write $\pi_{\gamma}^{I}$ for its representation map. 
Let $\gamma \in E_{\mathbb{C}, I}^{\kappa}$. For $w \in W_{0}$ set

$$
v_{w}^{I}(\gamma):=T_{w} \otimes_{\left(H_{I}(\kappa), \chi_{I, \gamma}\right)} 1 \in M_{I}(\gamma)
$$

The elements $v_{w}^{I}(\gamma)\left(w \in W_{0}\right)$ span $M_{I}(\gamma)$.

Let $W_{0, I} \subset W_{0}$ be the standard parabolic subgroup generated by the simple reflections $s_{i}(i \in I)$. Denote by $W_{0}^{I}$ the minimal coset representatives of $W_{0} / W_{0, I}$. If $w=u v \in W_{0}$ with $u \in W_{0}^{I}$ and $v \in W_{0, I}$ then

$$
v_{w}^{I}(\gamma)=\chi_{I, \gamma}\left(T_{v}\right) v_{u}^{I}(\gamma)
$$

The set $\left\{v_{w}^{I}(\gamma)\right\}_{w \in W_{0}^{I}}$ is a linear basis of the principal series module $M_{I}(\gamma)$.

In case of minimal principal series $(I=\emptyset)$ we omit $I$ from the notations. In particular we write $\pi_{\gamma}: H(\kappa) \rightarrow \operatorname{End}_{\mathbb{C}}(M(\gamma))$ for the corresponding representation map. Note that $\pi_{\gamma}$ is defined for all $\gamma \in E_{\mathbb{C}}$.

Let $\gamma \in E_{\mathbb{C}, I}^{\kappa}$. There exists a unique surjective intertwiner $\phi_{I, \gamma}: M(\gamma) \rightarrow M_{I}(\gamma)$ of $H(\kappa)$-modules satisfying for $w=u v \in W_{0}\left(u \in W_{0}^{I}, v \in W_{0, I}\right)$,

$$
\phi_{I, \gamma}\left(v_{w}(\gamma)\right)=v_{w}^{I}(\gamma)=\chi_{I, \gamma}\left(T_{v}\right) v_{u}^{I}(\gamma) .
$$

Clearly $\phi_{\emptyset, \gamma}=\operatorname{Id}_{M(\gamma)}$.

We need generic conditions on $\gamma \in E_{\mathbb{C}, I}^{\kappa}$ to ensure that the principal series module $M_{I}(\gamma)$ is calibrated (i.e., simultaneously diagonalisable for the action of the commuting operators $\left.Y^{v}(v \in \widetilde{\Lambda})\right)$, cf. [62, Sect. 2.5] and references therein. To describe the results we first need to introduce intertwiners for minimal principal series modules.

Let $\gamma \in E_{\mathbb{C}}$ and let $e \in W_{0}$ be the neutral element. Set $A_{e}^{u n n}(\gamma):=\operatorname{Id}_{M(\gamma)}$. For $1 \leq i \leq n$ define a linear map $A_{s_{i}}^{u n n}(\gamma): M\left(s_{i} \gamma\right) \rightarrow M(\gamma)$ by

$$
\begin{aligned}
A_{s_{i}}^{u n n}(\gamma) v_{\sigma}\left(s_{i} \gamma\right):= & q^{-\widetilde{\kappa}_{i}}\left(1-q^{2\left(\widetilde{\alpha}_{i}, \gamma\right)}\right) v_{\sigma s_{i}}(\gamma) \\
& +\left(D_{\widetilde{\alpha}_{i}}(\gamma)-q^{-2 \chi\left(-\sigma \widetilde{\alpha}_{i}\right) \widetilde{\kappa}_{i}}\left(1-q^{2\left(\widetilde{\alpha}_{i}, \gamma\right)}\right)\right) v_{\sigma}(\gamma)
\end{aligned}
$$

for $\sigma \in W_{0}$, where $\chi(\widetilde{\alpha})=1$ if $\widetilde{\alpha} \in \widetilde{R}_{0}^{-}$and $\chi(\widetilde{\alpha})=0$ if $\widetilde{\alpha} \in \widetilde{R}_{0}^{+}$, and with

$$
\begin{aligned}
D_{\widetilde{\alpha}}(\gamma) & :=\left(1-q^{-\widetilde{\kappa}_{\widetilde{\alpha}}-\widetilde{\kappa}_{2 \widetilde{\alpha}}+(\widetilde{\alpha}, \gamma)}\right)\left(1+q^{-\widetilde{\kappa}_{\widetilde{\alpha}}+\widetilde{\kappa}_{2 \widetilde{\alpha}}+(\widetilde{\alpha}, \gamma)}\right) \\
& =\left(1-\widetilde{a}_{\alpha}^{-1} q^{(\widetilde{\alpha}, \gamma)}\right)\left(1-\widetilde{b}_{\alpha}^{-1} q^{(\widetilde{\alpha}, \gamma)}\right)
\end{aligned}
$$

for $\alpha \in R_{0}$. Then $A_{s_{i}}^{u n n}(\gamma): M\left(s_{i} \gamma\right) \rightarrow M(\gamma)$ is an intertwiner of $H(\kappa)$-modules and

$$
A_{s_{i}}^{u n n}(\gamma) A_{s_{i}}^{u n n}\left(s_{i} \gamma\right)=D_{s_{i}}(\gamma) D_{s_{i}}\left(s_{i} \gamma\right) \operatorname{Id}_{M(\gamma)}
$$

for all $1 \leq i \leq n$. More generally, if $\sigma:=s_{i_{1}} s_{i_{2}} \cdots s_{i_{r}}$ is a reduced expression for $\sigma \in W_{0}$ then

$$
A_{\sigma}^{u n n}(\gamma):=A_{s_{i_{1}}}^{u n n}(\gamma) A_{s_{i_{2}}}^{u n n}\left(s_{i_{1}} \gamma\right) \cdots A_{s_{i_{r}}}^{u n n}\left(s_{i_{r-1}} \cdots s_{i_{2}} s_{i_{1}} \gamma\right)
$$

is independent of the choice of reduced expression and defines an intertwiner

$$
A_{\sigma}^{u n n}(\gamma): M\left(\sigma^{-1} \gamma\right) \rightarrow M(\gamma)
$$


of $H(\kappa)$-modules. It satisfies

$$
A_{\sigma}^{u n n}(\gamma) A_{\sigma^{-1}}^{u n n}\left(\sigma^{-1} \gamma\right)=D_{\sigma}(\gamma) D_{\sigma^{-1}}\left(\sigma^{-1} \gamma\right) \operatorname{Id}_{M(\gamma)}
$$

with

$$
D_{\sigma}(\gamma):=\prod_{\widetilde{\alpha} \in \widetilde{R}_{0}^{+} \cap \sigma \widetilde{R}_{0}^{-}} D_{\widetilde{\alpha}}(\gamma)
$$

Note that

$$
\begin{aligned}
& A_{\sigma \tau}^{u n n}(\gamma)=A_{\sigma}^{u n n}(\gamma) A_{\tau}^{u n n}\left(\sigma^{-1} \gamma\right), \\
& D_{\sigma \tau}^{u n n}(\gamma)=D_{\sigma}^{u n n}(\gamma) D_{\tau}^{u n n}\left(\sigma^{-1} \gamma\right)
\end{aligned}
$$

for $\sigma, \tau \in W_{0}$ satisfying $l(\sigma \tau)=l(\sigma)+l(\tau)$.

Set $\widetilde{R}_{0}^{I, \pm}:=\widetilde{R}_{0}^{ \pm} \cap \operatorname{span}\left\{\widetilde{\alpha}_{i}\right\}_{i \in I}$. Since

$$
W_{0}^{I}=\left\{\sigma \in W_{0} \mid \sigma\left(\widetilde{R}_{0}^{I,+}\right) \subseteq \widetilde{R}_{0}^{+}\right\}
$$

we actually have

$$
D_{\sigma^{-1}}(\gamma)=\prod_{\widetilde{\alpha} \in\left(\widetilde{R}_{0}^{+} \backslash \widetilde{R}_{0}^{I,+}\right) \cap \sigma^{-1} \widetilde{R}_{0}^{-}} D_{\widetilde{\alpha}}(\gamma) \quad \forall \sigma \in W_{0}^{I} .
$$

Proposition 3.4. Assume that $\gamma \in E_{\mathbb{C}, I}^{\kappa}$ satisfies $q^{2(\widetilde{\alpha}, \gamma)} \neq 1$ for all $\widetilde{\alpha} \in \widetilde{R}_{0}^{+} \backslash \widetilde{R}_{0}^{I,+}$. Set for $u \in W_{0}$,

$$
b_{u}^{u n n, I}(\gamma):=\phi_{I, \gamma}\left(A_{u}^{u n n}(\gamma) v_{e}\left(u^{-1} \gamma\right)\right) \in M_{I}(\gamma)
$$

Then

(1) For all $u \in W_{0}$ and all $v \in \widetilde{\Lambda}$ we have

$$
\pi_{\gamma}^{I}\left(Y^{v}\right) b_{u}^{u n n, I}(\gamma)=q^{-\left(v, u^{-1} \gamma\right)} b_{u}^{u n n, I}(\gamma)
$$

(2) $\left\{b_{\sigma^{-1}}^{\text {unn,I}}(\gamma)\right\}_{\sigma \in W_{0}^{I}}$ is a linear basis of $M_{I}(\gamma)$;

(3) $b_{\sigma^{-1}}^{u n n, I}(\gamma)=0$ for all $\sigma \in W_{0} \backslash W_{0}^{I}$.

Proof. (1) is trivial.

(2) By the definition of the unnormalized intertwiner we have for $\sigma \in W_{0}^{I}$

$$
b_{\sigma^{-1}}^{u n n, I}(\gamma)=\left(\prod_{\widetilde{\alpha} \in\left(\widetilde{R}_{0}^{+} \backslash \widetilde{R}_{0}^{I,+}\right) \cap \sigma^{-1} \widetilde{R}_{0}^{-}} q^{-\widetilde{\kappa} \widetilde{\alpha}}\left(1-q^{2(\widetilde{\alpha}, \gamma)}\right)\right) v_{\sigma}^{I}(\gamma)+\sum_{\tau \in W_{0}^{I}: \tau<\sigma} c_{\sigma, \tau} v_{\tau}^{I}(\gamma)
$$

for certain coefficients $c_{\sigma, \tau} \in \mathbb{C}$, where $\leq$ is the Bruhat order. By the assumption that $q^{2(\widetilde{\alpha}, \gamma)} \neq 1$ for all $\widetilde{\alpha} \in \widetilde{R}_{0}^{+} \backslash \widetilde{R}_{0}^{I,+}$ the leading coefficient is nonzero.

(3) Fix $\sigma \notin W_{0}^{I}$. Let $i \in I$ such that $\sigma=\sigma^{\prime} s_{i}$ and $l(\sigma)=l\left(\sigma^{\prime}\right)+1$ for some $i \in I$. Then

$$
A_{\sigma^{-1}}^{u n n}(\gamma)=A_{s_{i}}^{u n n}(\gamma) A_{\sigma^{\prime}-1}^{u n n}\left(s_{i} \gamma\right) .
$$

Hence it suffices to show that $\phi_{I, \gamma} \circ A_{s_{i}}^{u n n}(\gamma)$ is the zero map. 
Since $\gamma \in E_{\mathbb{C}, I}^{\kappa}$ we have $D_{\widetilde{\alpha}_{i}}(\gamma)=0$, hence by (3.3),

$$
\begin{aligned}
& A_{s_{i}}^{u n n}(\gamma) v_{\tau}\left(s_{i} \gamma\right)=q^{-\widetilde{\kappa}_{i}}\left(1-q^{2\left(\widetilde{\alpha}_{i}, \gamma\right)}\right) v_{\tau s_{i}}(\gamma) \\
& \quad-q^{-2 \chi\left(-\tau \widetilde{\alpha}_{i}\right) \widetilde{\kappa}_{i}}\left(1-q^{2\left(\widetilde{\alpha}_{i}, \gamma\right)}\right) v_{\tau}(\gamma), \quad \tau \in W_{0} .
\end{aligned}
$$

Suppose that $\tau \in W_{0}$ satisfies $\tau \widetilde{\alpha}_{i} \in \widetilde{R}_{0}^{+}\left(\Leftrightarrow l\left(\tau s_{i}\right)=l(\tau)+1\right)$. Then $v_{\tau s_{i}}^{I}(\gamma)=$ $q^{-\kappa_{i}} v_{\tau}^{I}(\gamma)=q^{-\widetilde{\kappa}_{i}} v_{\tau}^{I}(\gamma)$ and $\chi\left(-\tau \widetilde{\alpha}_{i}\right)=1$, hence $\phi_{I, \gamma}\left(A_{s_{i}}^{u n n}(\gamma) v_{\tau}\left(s_{i} \gamma\right)\right)=0$.

Suppose that $\tau \in W_{0}$ satisfies $\tau \widetilde{\alpha}_{i} \in \widetilde{R}_{0}^{-}\left(\Leftrightarrow l\left(\tau s_{i}\right)=l(\tau)-1\right)$. Then

$$
v_{\tau s_{i}}^{I}(\gamma)=q^{\kappa_{i}} v_{\tau}^{I}(\gamma)=q^{\widetilde{\kappa}_{i}} v_{\tau}^{I}(\gamma) \text { and } \chi\left(-\tau \widetilde{\alpha}_{i}\right)=0
$$

hence also in this case

$$
\phi_{I, \gamma}\left(A_{s_{i}}^{u n n}(\gamma) v_{\tau}\left(s_{i} \gamma\right)\right)=0
$$

Hence indeed $\phi_{I, \gamma} \circ A_{s_{i}}^{u n n}(\gamma)$ for $i \in I$ is the zero map.

Definition 3.5. Suppose that $\gamma \in E_{\mathbb{C}}$ satisfies $q^{2(\widetilde{\alpha}, \gamma)} \neq 1$ and $D_{\widetilde{\alpha}}(\gamma) \neq 0$ for all $\widetilde{\alpha} \in \widetilde{R}_{0}^{+}$. Let $\sigma \in W_{0}$. The normalised intertwiner $A_{\sigma}(\gamma): M\left(\sigma^{-1} \gamma\right) \rightarrow M(\gamma)$ is defined by

$$
A_{\sigma}(\gamma):=D_{\sigma}(\gamma)^{-1} A_{\sigma}^{u n n}(\gamma), \quad \sigma \in W_{0}
$$

Note that normalised intertwiners satisfy $A_{e}(\gamma)=\operatorname{Id}_{M(\gamma)}$ and

$$
A_{\sigma}(\gamma) A_{\tau}\left(\sigma^{-1} \gamma\right)=A_{\sigma \tau}(\gamma) \quad \forall \sigma, \tau \in W_{0}
$$

Let $\gamma \in E_{\mathbb{C}, I}^{\kappa}$ be generic. Note that $D_{\sigma^{-1}}(\gamma)=0$ if $\sigma \in W_{0}$ is such that $\widetilde{\alpha}_{i} \in \sigma^{-1} \widetilde{R}_{0}^{-}$ for some $i \in I$. Since this cannot happen if $\sigma \in W_{0}^{I}$ (see (3.5) and (3.6)), the normalised intertwiner $A_{\sigma^{-1}}(\gamma)$ is well defined for $\sigma \in W_{0}^{I}$. Combined with Proposition 3.4 we obtain the following corollary.

Corollary 3.6. Suppose that $\gamma \in E_{\mathbb{C}, I}^{\kappa}$ satisfies the (generic) conditions that $q^{2(\widetilde{\alpha}, \gamma)} \neq 1$ and $D_{\widetilde{\alpha}}(\gamma) \neq 0$ for all $\widetilde{\alpha} \in \widetilde{R}_{0}^{+} \backslash \widetilde{R}_{0}^{I,+}$. Then

$$
\begin{aligned}
b_{\sigma^{-1}}^{I}(\gamma) & :=D_{\sigma^{-1}}(\gamma)^{-1} b_{\sigma^{-1}}^{u n n, I}(\gamma) \\
& =\phi_{I, \gamma}\left(A_{\sigma^{-1}}(\gamma) v_{e}(\sigma \gamma)\right)
\end{aligned}
$$

is well defined for all $\sigma \in W_{0}^{I}$ and $\left\{b_{\sigma^{-1}}^{I}(\gamma)\right\}_{\sigma \in W_{0}^{I}}$ is a complex linear basis of $M_{I}(\gamma)$ satisfying

$$
\pi_{\gamma}^{I}\left(Y^{\nu}\right) b_{\sigma^{-1}}^{I}(\gamma)=q^{-(\nu, \sigma \gamma)} b_{\sigma^{-1}}^{I}(\gamma) \quad \forall v \in \widetilde{\Lambda}, \forall \sigma \in W_{0}^{I} .
$$


3.3. Quantum affine $K Z$ equations. Define for $a \in R^{\bullet}$ the meromorphic function $c_{a}(\mathbf{z} ; \kappa)$ in $\mathbf{z} \in E_{\mathbb{C}}$ by

$$
c_{a}(\mathbf{z} ; \kappa):=\frac{\left(1-q^{-\kappa_{a}-\kappa_{2 a}+a(\mathbf{z})}\right)\left(1+q^{-\kappa_{a}+\kappa_{2 a}+a(\mathbf{z})}\right)}{\left(1-q^{2 a(\mathbf{z})}\right)},
$$

where we view $a=\alpha^{(r)}=\mu_{\alpha} r c+\alpha$ as affine linear function on $E_{\mathbb{C}}$ by

$$
a(\mathbf{z}):=\mu_{\alpha} r+(\alpha, \mathbf{z}) .
$$

We omit the $\kappa$-dependence from the notation if no confusion is possible. We write $c_{j}(\mathbf{z}):=c_{\alpha^{(j)}}(\mathbf{z} ; \kappa)$ and $\widetilde{c}_{j}(\mathbf{z}):=c_{\widetilde{\alpha}^{(j)}}(\mathbf{z} ; \widetilde{\kappa})$ for $0 \leq j \leq n$. The following theorem generalizes Cherednik's [9] results on the Baxterization of affine Hecke algebra modules to the current setting (including the twisted, untwisted and nonreduced cases all at once). Its proof uses the affine intertwiners of the double affine Hecke algebra associated to the data $(D, \kappa)($ see $[66])$.

Theorem 3.7. Let $\pi: H(\kappa) \rightarrow \operatorname{End}_{\mathbb{C}}(V)$ be a representation. The following formulas

$$
\begin{array}{ll}
\left(\nabla^{V}\left(s_{j}\right) f\right)(\mathbf{z}):=\left(\frac{q^{-\kappa_{j}} \pi\left(T_{j}\right)+c_{j}(\mathbf{z})-q^{-2 \kappa_{j}}}{c_{j}(\mathbf{z})}\right) f\left(s_{j} \mathbf{z}\right), & 0 \leq j \leq n, \\
\left(\nabla^{V}(\varpi) f\right)(\mathbf{z}):=\pi\left(T_{\varpi}\right) f\left(\varpi^{-1} \mathbf{z}\right), & \varpi \in \Omega
\end{array}
$$

define a left $W$-action $\nabla^{V}$ on the space of $V$-valued meromorphic functions $f(\mathbf{z})$ in $\underset{\mathbf{z}}{\mathbf{\Lambda}} \in E_{\mathbb{C}}$, where we use the natural action of $W \simeq W_{0} \ltimes \widetilde{\Lambda}$ on $\mathbf{z} \in E_{\mathbb{C}}$ by reflections and $\widetilde{\Lambda}$-translations.

Note that $\left(\nabla^{V}(w) f\right)(\mathbf{z})=C_{w}^{V}(\mathbf{z}) f\left(w^{-1} \mathbf{z}\right)$ for $w \in W$, with $C_{w}^{V}(\mathbf{z}): V \rightarrow V$ a linear map depending meromorphically on $\mathbf{z} \in E_{\mathbb{C}}$ and satisfying the cocycle conditions $C_{e}^{V}(\mathbf{z})=\operatorname{Id}_{V}$ and

$$
C_{u v}^{V}(\mathbf{z})=C_{u}^{V}(\mathbf{z}) C_{v}^{V}\left(u^{-1} \mathbf{z}\right), \quad \forall u, v \in W .
$$

Definition 3.8. For a given representation $\pi: H(\kappa) \rightarrow \operatorname{End}_{\mathbb{C}}(V)$ we say that a meromorphic $V$-valued function $f(\mathbf{z})$ in $\mathbf{z} \in E_{\mathbb{C}}$ is a solution of the associated quantum affine $\mathrm{KZ}$ equations if

$$
\left(\nabla^{V}(\tau(\lambda)) f\right)(\mathbf{z})=f(\mathbf{z}) \quad \forall \lambda \in \widetilde{\Lambda} .
$$

We write $\operatorname{Sol}_{K Z}(V)=\operatorname{Sol}_{K Z}(V ; \kappa)$ for the solution space.

Note that $\operatorname{Sol}_{K Z}(V)$ is $\nabla^{V}\left(W_{0}\right)$-stable. Note furthermore that the quantum affine $\mathrm{KZ}$ equations form a compatible system of difference equations. Indeed, the quantum affine $\mathrm{KZ}$ equations can be written as

$$
C_{\tau(\lambda)}^{V}(\mathbf{z}) f(\mathbf{z}-\lambda)=f(\mathbf{z}) \quad \forall \lambda \in \widetilde{\Lambda} .
$$

It is apparent from this form of the quantum affine $\mathrm{KZ}$ equations that the cocycle values $C_{\tau(\lambda)}^{V}(\mathbf{z})$ play the role of transport operators. The compatibility of the difference equations (3.9) in terms of the transport operators reads

$$
C_{\tau(\lambda)}^{V}(\mathbf{z}) C_{\tau(\mu)}^{V}(\mathbf{z}-\lambda)=C_{\tau(\lambda+\mu)}^{V}(\mathbf{z})=C_{\tau(\mu)}^{V}(\mathbf{z}) C_{\tau(\lambda)}^{V}(\mathbf{z}-\mu) \quad \forall \lambda, \mu \in \widetilde{\Lambda},
$$


which is a direct consequence of the cocycle property of $\left\{C_{u}^{V}(\mathbf{z})\right\}_{u \in W}$. Finally, note that an intertwiner $\psi: V \rightarrow V^{\prime}$ of $H(\kappa)$-modules gives rise to a $W_{0}$-intertwiner $\operatorname{Sol}_{K Z}(V) \rightarrow \operatorname{Sol}_{K Z}\left(V^{\prime}\right)$. We will denote this map also by $\psi$.

Explicit expressions for the cocycle $C_{w}^{M(\gamma)}(\mathbf{z})(w \in W)$ are given as follows.

Proposition 3.9. Let $\gamma \in E_{\mathbb{C}}$. For $\sigma \in W_{0}$,

$$
\begin{aligned}
& C_{s_{0}}^{M(\gamma)}(\mathbf{z}) v_{\sigma}(\gamma)=\frac{q^{(\widetilde{\psi}, \sigma \gamma)} v_{s_{\psi} \sigma}(\gamma)}{q^{\kappa_{0}} c_{0}(\mathbf{z})}+\left(\frac{c_{0}(\mathbf{z})-q^{-2 \chi\left(\sigma^{-1} \widetilde{\psi}\right) \kappa_{0}}}{c_{0}(\mathbf{z})}\right) v_{\sigma}(\gamma), \\
& C_{s_{i}}^{M(\gamma)}(\mathbf{z}) v_{\sigma}(\gamma)=\frac{v_{s_{i} \sigma}(\gamma)}{q^{\kappa_{i}} c_{i}(\mathbf{z})}+\left(\frac{c_{i}(\mathbf{z})-q^{-2 \chi\left(-\sigma^{-1} \widetilde{\alpha}_{i}\right) \kappa_{i}}}{c_{i}(\mathbf{z})}\right) v_{\sigma}(\gamma), \\
& C_{\varpi}^{M(\gamma)}(\mathbf{z}) v_{\sigma}(\gamma)=q^{-\left(\varpi, w_{0} \sigma \gamma\right)} v_{v(\varpi)^{-1} \sigma}(\gamma)
\end{aligned}
$$

for $1 \leq i \leq n$ and $\varpi \in \Omega$, where $w_{0} \in W_{0}$ is the longest Weyl group element.

Proof. This follows from the explicit formulas for the action of the affine Hecke algebra generators on the basis $\left\{v_{\sigma}(\gamma)\right\}_{\sigma \in W_{0}}$ of $M(\gamma)$ (see, e.g., [64] and references therein).

3.4. Power series solutions. We first construct a basis consisting of power series solutions of $\operatorname{Sol}_{K Z}(M(\gamma))$. To determine the natural normalisation we need to investigate the dependence on $\gamma$. We identify $M(\gamma)$ as complex vector space with $\mathcal{V}:=\bigoplus_{\sigma \in W_{0}} \mathbb{C} v_{\sigma}$ via the linear isomorphism $M(\gamma) \stackrel{\sim}{\longrightarrow} \mathcal{V}$ satisfying $v_{\sigma}(\gamma) \mapsto v_{\sigma}$ for all $\sigma \in W_{0}$. Accordingly, we interpret the cocycle values $C_{w}^{M(\gamma)}(\mathbf{z}): M(\gamma) \rightarrow M(\gamma)(w \in W)$ as linear maps

$$
C_{w}(\mathbf{z}, \gamma): \mathcal{V} \rightarrow \mathcal{V}
$$

depending meromorphically on $(\mathbf{z}, \gamma) \in E_{\mathbb{C}} \times E_{\mathbb{C}}$. In particular, for all $\sigma \in W_{0}$,

$$
\begin{aligned}
C_{s_{0}}(\mathbf{z}, \gamma) v_{\sigma} & =\frac{q^{(\widetilde{\psi}, \sigma \gamma)} v_{s_{\psi} \sigma}}{q^{\kappa_{0}} c_{0}(\mathbf{z})}+\left(\frac{c_{0}(\mathbf{z})-q^{-2 \chi\left(\sigma^{-1} \widetilde{\psi}\right) \kappa_{0}}}{c_{0}(\mathbf{z})}\right) v_{\sigma}, \\
C_{s_{i}}(\mathbf{z}, \gamma) v_{\sigma} & =\frac{v_{s_{i} \sigma}}{q^{\kappa_{i}} c_{i}(\mathbf{z})}+\left(\frac{c_{i}(\mathbf{z})-q^{-2 \chi\left(-\sigma^{-1} \widetilde{\alpha}_{i}\right) \kappa_{i}}}{c_{i}(\mathbf{z})}\right) v_{\sigma}, \\
C_{\varpi}(\mathbf{z}, \gamma) v_{\sigma} & =q^{-\left(\varpi, w_{0} \sigma \gamma\right)} v_{v(\varpi)^{-1} \sigma}
\end{aligned}
$$

for $1 \leq i \leq n$ and $\varpi \in \Omega$.

For fixed $\gamma \in E_{\mathbb{C}}$, the solution space $\operatorname{Sol}_{K Z}(M(\gamma))$ thus identifies with the space of $\mathcal{V}$-valued meromorphic functions $f(\mathbf{z}, \gamma)$ in $\mathbf{z} \in E_{\mathbb{C}}$ satisfying

$$
C_{\tau(\lambda)}(\mathbf{z}, \gamma) f(\mathbf{z}-\lambda, \gamma)=f(\mathbf{z}, \gamma), \quad \forall \lambda \in \widetilde{\Lambda}
$$

which form half of the bispectral quantum KZ equations (see [50,51,63]); the other half is a compatible dual system of quantum affine $\mathrm{KZ}$ equations acting on $\gamma$.

Define the plane wave function by

$$
\mathcal{W}(\mathbf{z}, \gamma):=q^{\left(\widetilde{\rho}+w_{0} \mathbf{z}, \rho-\gamma\right)}
$$


where

$$
\rho:=\frac{1}{2} \sum_{\alpha \in R_{0}^{+}}\left(\kappa_{\alpha}+\kappa_{\alpha^{(1)}}\right) \widetilde{\alpha}^{\vee}, \quad \widetilde{\rho}:=\frac{1}{2} \sum_{\alpha \in R_{0}^{+}}\left(\widetilde{\kappa}_{\widetilde{\alpha}}+\widetilde{\kappa}_{\widetilde{\alpha}^{(1)}}\right) \alpha^{\vee} .
$$

In addition define $\mathcal{S}(\mathbf{z})=\mathcal{S}(\mathbf{z} ; \kappa)$ by

$$
\mathcal{S}(\mathbf{z}):=\prod_{\alpha \in R_{0}^{+}}\left(q_{\alpha}^{2} a_{\alpha}^{-1} q^{-(\alpha, \mathbf{z})}, q_{\alpha}^{2} b_{\alpha}^{-1} q^{-(\alpha, \mathbf{z})}, q_{\alpha}^{2} c_{\alpha}^{-1} q^{-(\alpha, \mathbf{z})}, q_{\alpha}^{2} d_{\alpha}^{-1} q^{-(\alpha, \mathbf{z})} ; q_{\alpha}^{2}\right)_{\infty}
$$

and $\widetilde{\mathcal{S}}(\gamma)=\widetilde{\mathcal{S}}(\gamma ; \widetilde{\kappa})$ by

$$
\widetilde{\mathcal{S}}(\gamma):=\prod_{\alpha \in R_{0}^{+}}\left(q_{\alpha}^{2} \widetilde{a}_{\alpha}^{-1} q^{-(\widetilde{\alpha}, \gamma)}, q_{\alpha}^{2} \widetilde{b}_{\alpha}^{-1} q^{-(\widetilde{\alpha}, \gamma)}, q_{\alpha}^{2} \widetilde{c}_{\alpha}^{-1} q^{-(\widetilde{\alpha}, \gamma)}, q_{\alpha}^{2} \widetilde{d}_{\alpha}^{-1} q^{-(\widetilde{\alpha}, \gamma)} ; q_{\alpha}^{2}\right)_{\infty} .
$$

Note that $\widetilde{\rho}$ and $\widetilde{\mathcal{S}}$ are obtained from $\rho$ and $\mathcal{S}$ by replacing the initial data $(D, \kappa)$ by $(\widetilde{D}, \widetilde{\kappa})$. The zero locus of the holomorphic function $\mathcal{S}(\mathbf{z})$ will give the singularities of the power series solutions of the quantum affine KZ equations (3.10).

Write $Q_{+}:=\mathbb{Z}_{\geq 0} R_{0}^{+}$. The following theorem comprises the results of [64, Sect. 3]. Special cases have been proven before in $[50,51]$.

Theorem 3.10. There exist unique $\mathcal{V}$-valued holomorphic functions $\Gamma_{\mu}(\gamma)$ in $\gamma \in E_{\mathbb{C}}$ $\left(\mu \in Q_{+}\right)$such that

(1) $\Gamma_{0}(\gamma)=\left(\prod_{\alpha \in R_{0}^{+}}\left(q_{\alpha}^{2} q^{-2(\widetilde{\alpha}, \gamma)} ; q_{\alpha}^{2}\right)\right) v_{w_{0}}$;

(2) the $\mathcal{V}$-valued series

$$
\Psi(\mathbf{z}, \gamma):=\sum_{\mu \in Q_{+}} \Gamma_{\mu}(\gamma) q^{-(\mu, \mathbf{z})}
$$

converges normally for $(\mathbf{z}, \gamma)$ in compacta of $E_{\mathbb{C}} \times E_{\mathbb{C}}$;

(3) the $\mathcal{V}$-valued meromorphic function $\Phi(\mathbf{z}, \gamma)=\Phi(\mathbf{z}, \gamma ; \kappa)$ in $(\mathbf{z}, \gamma) \in E_{\mathbb{C}} \times E_{\mathbb{C}}$ defined by

$$
\Phi(z, \gamma):=\frac{\mathcal{W}(z, \gamma)}{\mathcal{S}(\mathbf{z}) \widetilde{\mathcal{S}}(\gamma)} \Psi(\mathbf{z}, \gamma)
$$

satisfies

$$
C_{\tau(\lambda)}(\mathbf{z}, \gamma) \Phi(\mathbf{z}-\lambda, \gamma)=\Phi(\mathbf{z}, \gamma) \quad \forall \lambda \in \tilde{\Lambda}
$$

If $\widetilde{\Phi}$ denotes the $\mathcal{V}$-valued function $\Phi$ with initial data $(D, \kappa)$ replaced by $(\widetilde{D}, \widetilde{\kappa})$, then

$$
\widetilde{\Phi}(\gamma, \mathbf{z})=\Phi(\mathbf{z}, \gamma) .
$$

Remark 3.11. It follows from (3.12) and (3.13) that $\Phi(\mathbf{z}, \gamma)$ is a solution of dual system of quantum $\mathrm{KZ}$ equations acting as difference equations on $\gamma$. The quantum affine $\mathrm{KZ}$ equations (3.10) together with the dual quantum $\mathrm{KZ}$ equations are the bispectral quantum $\mathrm{KZ}$ equations from [50,51,64]. In [64] the power series solution $\Phi(\mathbf{z}, \gamma)$ is first constructed and characterised as power series solution of the bispectral quantum KZ equations. The other properties, such as the duality property (3.13), the singularities and the normalisation, are subsequently derived. 
Definition 3.12. We call $\gamma \in E_{\mathbb{C}, I}^{\kappa}$ generic if

(1) $q^{2(\widetilde{\alpha}, \gamma)} \neq 1$ for all $\alpha \in R_{0}^{+} \backslash R_{0}^{I,+}$;

(2) $q^{(\widetilde{\alpha}, \gamma)} \notin\left\{\widetilde{a}_{\alpha}, \widetilde{b}_{\alpha}\right\}$ for all $\alpha \in R_{0}^{+} \backslash R_{0}^{I,+}$;

(3) $q^{2(\widetilde{\alpha}, \gamma)} \notin q_{\alpha}^{2 \mathbb{Z}_{>0}}$ for all $\alpha \in R_{0} \backslash R_{0}^{I,-}$;

(4) $q^{(\widetilde{\alpha}, \gamma)} \notin\left\{\widetilde{a}_{\alpha}^{-1} q_{\alpha}^{2 \mathbb{Z}_{>0}}, \widetilde{b}_{\alpha}^{-1} q_{\alpha}^{2 \mathbb{Z}_{>0}}, \widetilde{c}_{\alpha}^{-1} q_{\alpha}^{2 \mathbb{Z}_{>0}}, \widetilde{d}_{\alpha}^{-1} q_{\alpha}^{2 \mathbb{Z}_{>0}}\right\}$ for all $\alpha \in R_{0} \backslash R_{0}^{I,-}$.

Note that these are indeed generic conditions on $\gamma \in E_{\mathbb{C}, I}^{\kappa}$ since

$$
E_{\mathbb{C}, I}^{\kappa} \subseteq\left\{\gamma \in E_{\mathbb{C}} \mid q^{\left(\widetilde{\alpha}_{i}, \gamma\right)}=\widetilde{a}_{i} \quad \forall i \in I\right\}
$$

The condition (1) is to ensure that $\left\{b_{\sigma^{-1}}^{u n n, I}\right\}_{\sigma \in W_{0}^{I}}$ is a well defined basis of $M_{I}(\gamma)$. Condition (2) ensures that $D_{\widetilde{\alpha}}(\gamma) \neq 0$ for all $\alpha \in R_{0}^{+} \backslash R_{0}^{I,+}$, hence the unnormalised basis $\left\{b_{\sigma^{-1}}^{u n n, I}(\gamma)\right\}_{\sigma \in W_{0}^{I}}$ of $M_{I}(\gamma)$ can be turned into the normalised basis $\left\{b_{\sigma^{-1}}^{I}(\gamma)\right\}_{\sigma \in W_{0}^{I}}$ and the normalised intertwiners $A_{\sigma^{-1}}(\gamma)$ are well defined for all $\sigma \in W_{0}^{I}$ (see Corollary 3.6). Condition (3) ensures that the leading coefficient $\Gamma_{0}(\sigma \gamma)$ in the power series solution of the quantum affine $\mathrm{KZ}$ equations is nonzero for all $\sigma \in W_{0}^{I}$. Finally, condition (4) ensures that $\widetilde{\mathcal{S}}(\sigma \gamma) \neq 0$ for all $\sigma \in W_{0}^{I}$.

For $\gamma \in E_{\mathbb{C}, I}^{\kappa}$ we write $\phi_{I, \gamma}^{\mathcal{V}}: \mathcal{V} \rightarrow M_{I}(\gamma)$ for the surjective linear map defined by

$$
\phi_{I, \gamma}^{\mathcal{V}}\left(v_{w}\right):=\chi_{I, \gamma}\left(T_{v}\right) v_{u}^{I}(\gamma)
$$

for $w=u v \in W_{0}$ with $u \in W_{0}^{I}$ and $v \in W_{0, I}$. We write $\phi_{\gamma}^{\mathcal{V}}:=\phi_{\emptyset, \gamma}^{\mathcal{V}}$. Note that

$$
\phi_{I, \gamma}^{\mathcal{V}}=\phi_{I, \gamma} \circ \phi_{\gamma}^{\mathcal{V}}
$$

Write $F$ for the field of $\widetilde{\Lambda}$-translation invariant meromorphic functions on $E_{\mathbb{C}}$.

Proposition 3.13. For generic $\gamma \in E_{\mathbb{C}, I}^{\kappa}$ and $\sigma \in W_{0}^{I}$ define

$$
\Phi_{\sigma^{-1}}^{I}(\mathbf{z}, \gamma):=\phi_{I, \gamma}\left(A_{\sigma^{-1}}(\gamma) \phi_{\sigma \gamma}^{\mathcal{V}}(\Phi(\mathbf{z}, \sigma \gamma))\right)
$$

Then $\left\{\Phi_{\sigma^{-1}}^{I}(\cdot, \gamma)\right\}_{\sigma \in W_{0}^{I}}$ is a F-linear basis of $\operatorname{Sol}_{K Z}\left(M_{I}(\gamma)\right)$. Furthermore,

$$
\Phi_{\sigma^{-1}}^{I}(\mathbf{z}, \gamma)=\frac{\mathcal{W}(\mathbf{z}, \sigma \gamma)}{\mathcal{S}(\mathbf{z}) \widetilde{\mathcal{S}}(\sigma \gamma)} \sum_{\alpha \in Q_{+}} \Gamma_{\alpha, \sigma^{-1}}^{I}(\gamma) q^{-(\alpha, \mathbf{z})}
$$

with the $M_{I}(\gamma)$-valued power series converging normally for $\mathbf{z}$ in compact of $E_{\mathbb{C}}$ and with leading coefficient

$$
\Gamma_{0, \sigma^{-1}}^{I}(\gamma)=\left(\prod_{\alpha \in R_{0}^{+}}\left(q_{\alpha}^{2} q^{-2(\widetilde{\alpha}, \sigma \gamma)} ; q_{\alpha}^{2}\right)_{\infty}\right) \pi_{\gamma}^{I}\left(T_{w_{0}}\right) b_{\sigma^{-1}}^{I}(\gamma)
$$


Proof. Since $\gamma \in E_{\mathbb{C}, I}^{\kappa}$ is generic, the $\Phi_{\sigma^{-1}}^{I}(\cdot, \gamma)\left(\sigma \in W_{0}^{I}\right)$ are well defined $M_{I}(\gamma)$ valued meromorphic functions satisfying the quantum affine $\mathrm{KZ}$ equations

$$
C_{\tau(\lambda)}^{M_{I}(\gamma)}(\mathbf{z}) \Phi_{\sigma^{-1}}^{I}(\mathbf{z}-\lambda)=\Phi_{\sigma^{-1}}^{I}(\mathbf{z}), \quad \lambda \in \tilde{\Lambda}
$$

They are $F$-linearly independent because their leading coefficients $\pi_{\gamma}^{I}\left(T_{w_{0}}\right) b_{\sigma-1}^{I}(\gamma)$ $\left(\sigma \in W_{0}^{I}\right)$ form a linear basis of $M_{I}(\gamma)$. The solutions $\Phi_{\sigma^{-1}}^{I}(\cdot, \gamma)\left(\sigma \in W_{0}^{I}\right)$ can be used to define a fundamental matrix solution of the quantum affine $\mathrm{KZ}$ equations (3.16), from which it follows that

$$
\operatorname{Dim}_{F}\left(\operatorname{Sol}_{K Z}\left(M_{I}(\gamma)\right)\right)=\operatorname{Dim}_{\mathbb{C}}\left(M_{I}(\gamma)\right) .
$$

Hence the $\Phi_{\sigma^{-1}}^{I}(\cdot, \gamma)\left(\sigma \in W_{0}^{I}\right)$ form a $F$-linear basis of $\operatorname{Sol}_{K Z}\left(M_{I}(\gamma)\right)$ (cf. [51, Sect. 5.6], where the proof is discussed in detail for the initial data of type $A$ and $I=\emptyset$ ).

3.5. The connection problem and its solution. Fix generic $\gamma \in E_{\mathbb{C}, I}^{\kappa}$. There exists unique coefficients

$$
m_{\tau_{1}, \tau_{2}}^{I, \sigma}(\cdot, \gamma) \in F \quad\left(\sigma \in W_{0} ; \tau_{1}, \tau_{2} \in W_{0}^{I}\right)
$$

such that

$$
\nabla^{M_{I}(\gamma)}(\sigma) \Phi_{\tau_{2}^{-1}}^{I}(\cdot, \gamma)=\sum_{\tau_{1} \in W_{0}^{I}} m_{\tau_{1}, \tau_{2}}^{I, \sigma}(\cdot, \gamma) \Phi_{\tau_{1}^{-1}}^{I}(\cdot, \gamma)
$$

Definition 3.14. We call the $\# W_{0}^{I} \times \# W_{0}^{I}$-matrices

$$
M^{I, \sigma}(\cdot, \gamma)=\left(m_{\tau_{1}, \tau_{2}}^{I, \sigma}(\cdot \gamma)\right)_{\tau_{1}, \tau_{2} \in W_{0}^{I}}, \quad \sigma \in W_{0}
$$

with coefficients in $F$ the connection matrices of the quantum afffine $\mathrm{KZ}$ equations associated to the initial data $(D, \kappa)$ and the principal series representation $M_{I}(\gamma)$.

The following cocycle property is immediate:

$$
\begin{aligned}
M^{I, \sigma \sigma^{\prime}}(\mathbf{z}, \gamma) & =M^{\sigma}(\mathbf{z}, \gamma) M^{\sigma^{\prime}}\left(\sigma^{-1} \mathbf{z}, \gamma\right), \quad \sigma, \sigma^{\prime} \in W_{0}, \\
M^{I, e}(\mathbf{z}, \gamma) & =\text { Id. }
\end{aligned}
$$

To explicitly compute the connection cocycle $\left\{M^{I, \sigma}(\mathbf{z}, \gamma)\right\}_{\sigma \in W_{0}}$ it thus suffices to compute $M^{I, s_{i}}(\mathbf{z}, \gamma)(1 \leq i \leq n)$.

To state the result we first need to introduce some more notations. For $i \in\{1, \ldots, n\}$ let $i^{*} \in\{1, \ldots, n\}$ be the index such that

$$
-w_{0}\left(\alpha_{i}\right)=\alpha_{i} \text {. }
$$

For $\sigma, \sigma^{\prime} \in W_{0}$ let $\delta_{\sigma, \sigma^{\prime}}$ be equal to one if $\sigma=\sigma^{\prime}$ and equal to zero otherwise. For a finite root $\alpha \in R_{0}$ write

$$
\mathfrak{e}_{\alpha}(x, y):=q^{-\frac{1}{2 \mu_{\alpha}}\left(\kappa_{\alpha}+\kappa_{2 \alpha}-x\right)\left(\kappa_{\alpha}+\kappa_{\alpha}(1)-y\right)} \frac{\theta\left(\widetilde{a}_{\alpha} q^{y}, \widetilde{b}_{\alpha} q^{y}, \widetilde{c}_{\alpha} q^{y}, d_{\alpha} q^{y-x} / \widetilde{a}_{\alpha} ; q_{\alpha}^{2}\right)}{\theta\left(q^{2 y}, d_{\alpha} q^{-x} ; q_{\alpha}^{2}\right)} .
$$

We write $\widetilde{\mathfrak{e}}_{\alpha}(x, y)\left(\alpha \in R_{0}\right)$ for its dual version,

$$
\widetilde{\mathfrak{e}}_{\alpha}(x, y):=q^{-\frac{1}{2 \mu_{\alpha}}\left(\kappa_{\alpha}+\kappa_{\alpha}(1)-x\right)\left(\kappa_{\alpha}+\kappa_{2 \alpha}-y\right)} \frac{\theta\left(a_{\alpha} q^{y}, b_{\alpha} q^{y}, c_{\alpha} q^{y}, \widetilde{d}_{\alpha} q^{y-x} / a_{\alpha} ; q_{\alpha}^{2}\right)}{\theta\left(q^{2 y}, \widetilde{d}_{\alpha} q^{-x} ; q_{\alpha}^{2}\right)} .
$$


Theorem 3.15. Fix generic $\gamma \in E_{\mathbb{C}, I}^{\kappa}$ satisfying the additional conditions

$$
q^{2(\widetilde{\beta}, \gamma)} \notin q_{\beta}^{2 \mathbb{Z}} \quad \forall \beta \in R_{0}
$$

Let $i \in\{1, \ldots, n\}$ and $\tau_{2} \in W_{0}^{I}$.

(1) If $s_{i} * \tau_{2} \notin W_{0}^{I}$ then

$$
m_{\tau_{1}, \tau_{2}}^{I, s_{i}}(\cdot, \gamma)=\delta_{\tau_{1}, \tau_{2}} \quad \forall \tau_{1} \in W_{0}^{I}
$$

(2) If $s_{i *} \tau_{2} \in W_{0}^{I}$ then

$$
m_{\tau_{1}, \tau_{2}}^{I, s_{i}}(\cdot, \gamma) \equiv 0 \quad \text { if } \tau_{1} \notin\left\{\tau_{2}, s_{i *} \tau_{2}\right\}
$$

and

$$
\begin{aligned}
m_{\tau_{2}, \tau_{2}}^{I, s_{i}}(\mathbf{z}, \gamma) & =\frac{\mathfrak{e}_{\alpha_{i}}\left(\left(\alpha_{i}, \mathbf{z}\right),\left(\widetilde{\alpha}_{i^{*}}, \tau_{2} \gamma\right)\right)-\widetilde{\mathfrak{e}}_{\alpha_{i}}\left(\left(\widetilde{\alpha}_{i^{*}}, \tau_{2} \gamma\right),\left(\alpha_{i}, \mathbf{z}\right)\right)}{\widetilde{\mathfrak{e}}_{\alpha_{i}}\left(\left(\widetilde{\alpha}_{i^{*}}, \tau_{2} \gamma\right),-\left(\alpha_{i}, \mathbf{z}\right)\right)}, \\
m_{s_{i} * \tau_{2}, \tau_{2}}^{I, s_{i}}(\mathbf{z}, \gamma) & =\frac{\mathfrak{e}_{\alpha_{i}}\left(\left(\alpha_{i}, \mathbf{z}\right),-\left(\widetilde{\alpha}_{i^{*}}, \tau_{2} \gamma\right)\right)}{\widetilde{\mathfrak{e}}_{\alpha_{i}}\left(\left(\widetilde{\alpha}_{i^{*}}, \tau_{2} \gamma\right),-\left(\alpha_{i}, \mathbf{z}\right)\right)} .
\end{aligned}
$$

Remark 3.16. An alternative formulation of Theorem 3.15 is as follows. Suppose that $\gamma \in E_{\mathbb{C}, I}^{\kappa}$ is generic and that (3.18) is valid. Let $i \in\{1, \ldots, n\}$ and $\tau_{2} \in W_{0}^{I}$. If $s_{i} * \tau_{2} \notin W_{0}^{I}$ then

$$
\nabla^{M_{I}(\gamma)}\left(s_{i}\right) \Phi_{\tau_{2}^{-1}}^{I}(\cdot, \gamma)=\Phi_{\tau_{2}^{-1}}^{I}(\cdot, \gamma)
$$

if $s_{i} * \tau_{2} \in W_{0}^{I}$ then

$$
\nabla^{M_{I}(\gamma)}\left(s_{i}\right) \Phi_{\tau_{2}^{-1}}^{I}(\cdot, \gamma)=m_{\tau_{2}, \tau_{2}}^{I, s_{i}}(\cdot, \gamma) \Phi_{\tau_{2}^{-1}}^{I}(\cdot, \gamma)+m_{s_{i}^{*} \tau_{2}, \tau_{2}}^{I, s_{i}}(\cdot, \gamma) \Phi_{\tau_{2}^{-1} s_{i^{*}}}^{I}(\cdot, \gamma)
$$

with the coefficients $m_{\tau_{2}, \tau_{2}}^{I, s_{i}}(\cdot, \gamma), m_{s_{i} * \tau_{2}, \tau_{2}}^{I, s_{i}}(\cdot, \gamma) \in F$ explicitly given by (3.19).

Proof. In [64, Thm. 1.6] the connection problem for the bispectral problem of the Ruijsenaars-Macdonald-Koornwinder-Cherednik difference operators associated to $(D, \kappa)$ is solved. Through the difference Cherednik-Matsuo correspondence it is equivalent to the solution of the connection problem for the bispectral quantum KZ equations associated to $(D, \kappa)$ (cf. the proof of [64, Thm. 1.5]). In our notations it gives the following. View the normalised intertwiners $A_{\tau}(\xi)\left(\tau \in W_{0}\right)$ as linear operators on $\mathcal{V}$ depending meromorphically on $\xi \in E_{\mathbb{C}}$ by identifying $\mathcal{V} \simeq M(\xi)$ as vector spaces through $\phi_{\xi} \mathcal{V}$. Then for $1 \leq i \leq n$ and $\tau_{2} \in W_{0}$,

$$
\begin{aligned}
C_{s_{i}}(\mathbf{z}, \xi)\left(A_{\tau_{2}^{-1}}(\xi) \Phi\left(s_{i} \mathbf{z}, \tau_{2} \xi\right)\right)= & n_{\tau_{2}, \tau_{2}}^{s_{i}}(\mathbf{z}, \xi)\left(A_{\tau_{2}^{-1}}(\xi) \Phi\left(\mathbf{z}, \tau_{2} \xi\right)\right) \\
& +n_{s_{i} * \tau_{2}, \tau_{2}}^{s_{i}}(\mathbf{z}, \xi)\left(A_{\tau_{2}^{-1} s_{i} *}(\xi) \Phi\left(\mathbf{z}, s_{i} * \tau_{2} \xi\right)\right)
\end{aligned}
$$


as $\mathcal{V}$-valued meromorphic functions in $(\mathbf{z}, \xi) \in E_{\mathbb{C}} \times E_{\mathbb{C}}$, with $n_{\tau_{1}, \tau_{2}}^{s_{i}}$ (which is denoted by $m_{\tau_{1}^{-1}, \tau_{2}^{-1}}^{s_{i}}$ in [64]) given explicitly by

$$
\begin{aligned}
& n_{\tau_{2}, \tau_{2}}^{s_{i}}(\mathbf{z}, \xi)=\frac{\mathfrak{e}_{\alpha_{i}}\left(\left(\alpha_{i}, \mathbf{z}\right),\left(\widetilde{\alpha}_{i^{*}}, \tau_{2} \xi\right)\right)-\widetilde{\mathfrak{e}}_{\alpha_{i}}\left(\left(\widetilde{\alpha}_{i^{*}}, \tau_{2} \xi\right),\left(\alpha_{i}, \mathbf{z}\right)\right)}{\widetilde{\mathfrak{e}}_{\alpha_{i}}\left(\left(\widetilde{\alpha}_{i^{*}}, \tau_{2} \xi\right),-\left(\alpha_{i}, \mathbf{z}\right)\right)}, \\
& n_{s_{i *}, \tau_{2}}^{s_{i}}(\mathbf{z}, \xi)=\frac{\mathfrak{e}_{\alpha_{i}}\left(\left(\alpha_{i}, \mathbf{z}\right),-\left(\widetilde{\alpha}_{i^{*}}, \tau_{2} \xi\right)\right)}{\widetilde{\mathfrak{e}}_{\alpha_{i}}\left(\left(\widetilde{\alpha}_{i^{*}}, \tau_{2} \xi\right),-\left(\alpha_{i}, \mathbf{z}\right)\right)} .
\end{aligned}
$$

By the additional generic conditions (3.18) on $\gamma \in E_{\mathbb{C}, I}^{\kappa}$, we can specialise $\xi$ in the coefficients $n_{\tau_{2}, \tau_{2}}^{s_{i}}(\cdot, \xi)$ and $n_{s_{i} * \tau_{2}, \tau_{2}}^{s_{i}}(\cdot, \xi)$ to $\gamma$ to get coefficients $n_{\tau_{2}, \tau_{2}}^{s_{i}}(\cdot, \gamma), n_{s_{i} * \tau_{2}, \tau_{2}}^{s_{i}}(\cdot, \gamma) \in$ $F$. We consider now two cases.

Case 1: $s_{i} * \tau_{2} \in W_{0}^{I}$.

Then in (3.20) we can specialise $\xi$ to $\gamma$ and apply $\phi_{I, \gamma}^{\mathcal{V}}$ to obtain

$$
C_{s_{i}}^{M_{I}(\gamma)}(\mathbf{z}) \Phi_{\tau_{2}^{-1}}^{I}\left(s_{i} \mathbf{z}, \gamma\right)=n_{\tau_{2}, \tau_{2}}^{s_{i}}(\mathbf{z}, \gamma) \Phi_{\tau_{2}^{-1}}^{I}(\mathbf{z}, \gamma)+n_{s_{i} * \tau_{2}, \tau_{2}}^{s_{i}}(\mathbf{z}, \gamma) \Phi_{\tau_{2}^{-1} s_{i} *}^{I}(\mathbf{z}, \gamma)
$$

as identity between $M_{I}(\gamma)$-valued meromorphic functions in $\mathbf{z} \in E_{\mathbb{C}}$, cf. Proposition 3.13. This proves (2).

Case 2: $s_{i} * \tau_{2} \notin W_{0}^{I}$. Then we rewrite (3.20) as

$$
\begin{aligned}
& C_{s_{i}}(\mathbf{z}, \xi)\left(A_{\tau_{2}^{-1}}(\xi) \Phi\left(s_{i} \mathbf{z}, \tau_{2} \xi\right)\right)=n_{\tau_{2}, \tau_{2}}^{s_{i}}(\mathbf{z}, \xi)\left(A_{\tau_{2}^{-1}}(\xi) \Phi\left(\mathbf{z}, \tau_{2} \xi\right)\right) \\
& \quad+G(\mathbf{z}, \xi)\left(\widetilde{\mathcal{S}}\left(s_{i^{*}} \tau_{2} \xi\right) A_{\tau_{2}^{-1} s_{i^{*}}}^{u n n}(\xi) \Phi\left(\mathbf{z}, s_{i} * \tau_{2} \xi\right)\right)
\end{aligned}
$$

with

$$
G(\mathbf{z}, \xi):=\frac{n_{s_{i * \tau_{2}, \tau_{2}}}^{s_{i}}(\mathbf{z}, \xi)}{\widetilde{\mathcal{S}}\left(s_{i^{*}} \tau_{2} \xi\right) D_{\tau_{2}^{-1} s_{i *}}(\xi)} .
$$

We first show that $G(\mathbf{z}, \xi)$ is regular at $\xi=\gamma$. Since $\tau_{2} \in W_{0}^{I}$ and $s_{i} * \tau_{2} \notin W_{0}^{I}$ we have $\alpha_{i *}=\tau_{2}(\beta)$ for some $\beta \in R_{0}^{I}$. So $s_{\beta} \in W_{0}^{I}$, hence

$$
l\left(s_{i} * \tau_{2}\right)=l\left(\tau_{2} s_{\beta}\right)=l\left(\tau_{2}\right)+l\left(s_{\beta}\right) .
$$

Consequently $\beta=\alpha_{j}$ for some $j \in I$ and $l\left(s_{i} * \tau_{2}\right)=l\left(\tau_{2} s_{j}\right)=l\left(\tau_{2}\right)+1$. Hence we have

$$
\begin{aligned}
D_{\tau_{2}^{-1} s_{i *}}(\xi) & =D_{\tau_{2}^{-1}}(\xi) D_{s_{i} *}\left(\tau_{2} \xi\right) \\
& =D_{\tau_{2}^{-1}}(\xi) D_{\alpha_{j}}(\xi) \\
& =D_{\tau_{2}^{-1}}(\xi)\left(1-\widetilde{a}_{j}^{-1} q^{\left(\widetilde{\alpha}_{j}, \xi\right)}\right)\left(1-\widetilde{b}_{j}^{-1} q^{\left(\widetilde{\alpha}_{j}, \xi\right)}\right)
\end{aligned}
$$

by (3.4), and $D_{\tau_{2}^{-1}}(\gamma) \neq 0$ in view of the generic conditions on $\gamma \in E_{\mathbb{C}, I}^{\kappa}$. Note though that $\left(1-\widetilde{a}_{j}^{-1} q^{\left(\widetilde{\alpha}_{j}, \xi\right)}\right)$ will be zero when specialising $\xi$ to $\gamma \in E_{\mathbb{C}, I}^{\kappa}$. We will show though in a moment that this factor also occurs as factor in the connection coefficient $n_{s_{i} * \tau_{2}, \tau_{2}}^{s_{i}}(\mathbf{z}, \xi)$, hence they will cancel each other out in (3.22). 
Observe that

$$
\begin{aligned}
\widetilde{\mathcal{S}}\left(s_{i} * \tau_{2} \xi\right) & =\widetilde{\mathcal{S}}^{r e m}(\xi)\left(q_{j}^{2} \widetilde{a}_{j}^{-1} q^{\left(\widetilde{\alpha}_{j}, \xi\right)}, q_{j}^{2} \widetilde{b}_{j}^{-1} q^{\left(\widetilde{\alpha}_{j}, \xi\right)}, q_{j}^{2} \widetilde{c}_{j}^{-1} q^{\left(\widetilde{\alpha}_{j}, \xi\right)}, q_{j}^{2} \widetilde{d}_{j}^{-1} q^{\left(\widetilde{\alpha}_{j}, \xi\right)} ; q_{j}^{2}\right)_{\infty}, \\
\widetilde{\mathcal{S}}^{r e m}(\xi) & :=\prod_{\alpha \in \tau_{2}^{-1}\left(R_{0}^{+}\right) \backslash\left\{\alpha_{j}\right\}}\left(q_{\alpha}^{2} \widetilde{a}_{\alpha}^{-1} q^{(\widetilde{\alpha}, \xi)}, q_{\alpha}^{2} \widetilde{b}_{\alpha}^{-1} q^{(\widetilde{\alpha}, \xi)}, q_{\alpha}^{2} \widetilde{c}_{\alpha}^{-1} q^{(\widetilde{\alpha}, \xi)}, q_{\alpha}^{2} \widetilde{d}_{\alpha}^{-1} q^{(\widetilde{\alpha}, \xi)} ; q_{\alpha}^{2}\right)_{\infty}
\end{aligned}
$$

and $\widetilde{\mathcal{S}}^{r e m}(\gamma) \neq 0$ by the generic conditions on $\gamma \in E_{\mathbb{C}, I}^{\kappa}$. The connection coefficient $n_{s_{i} * \tau_{2}, \tau_{2}}^{s_{i}}(\mathbf{z}, \xi)$ decouples,

$$
n_{s_{i} * \tau_{2}, \tau_{2}}^{s_{i}}(\mathbf{z}, \xi)=\frac{p_{\widetilde{\alpha}_{j}}\left(-\left(\widetilde{\alpha}_{j}, \xi\right)\right)}{p_{\alpha_{i}}\left(-\left(\alpha_{i}, \mathbf{z}\right)\right)}
$$

with

$$
\left.p_{\alpha}(x):=\frac{\theta\left(a_{\alpha} q^{x}, b_{\alpha} q^{x}, c_{\alpha} q^{x}, d_{\alpha} q^{x} ; q_{\alpha}^{2}\right)}{\theta\left(q^{2 x} ; q_{\alpha}^{2}\right)} q^{\frac{1}{\mu_{\alpha}}\left(\kappa_{\alpha}+\kappa_{\alpha}(1)\right.}\right) x
$$

and with $\widetilde{p}_{\widetilde{\alpha}}(x)$ its dual version

$$
\widetilde{p}_{\widetilde{\alpha}}(x):=\frac{\theta\left(\widetilde{a}_{\alpha} q^{x}, \widetilde{b}_{\alpha} q^{x}, \widetilde{c}_{\alpha} q^{x}, \widetilde{d}_{\alpha} q^{x} ; q_{\alpha}^{2}\right)}{\theta\left(q^{2 x} ; q_{\alpha}^{2}\right)} q^{\frac{1}{\mu_{\alpha}}\left(\widetilde{\kappa}_{\widetilde{\alpha}}+\widetilde{\kappa}_{\widetilde{\alpha}(1)}\right) x}
$$

(the decoupling formula in Remark 2.19(ii) is a special case). Combining these observations we conclude that the coefficient $G(\mathbf{z}, \xi)$ (see (3.22)) can be rewritten as

$$
\begin{aligned}
& G(\mathbf{z}, \xi)=\frac{\widetilde{a}_{j} \widetilde{b}_{j}}{p_{i}\left(-\left(\alpha_{i}, \mathbf{z}\right)\right) D_{\tau_{2}^{-1}}(\xi) \widetilde{\mathcal{S}}^{r e m}(\xi) \theta\left(q^{-2\left(\widetilde{\alpha}_{j}, \xi\right)} ; q_{j}^{2}\right)} q^{-2\left(\widetilde{\alpha}_{j}, \xi\right)-\frac{1}{\mu_{j}} \widetilde{\kappa}_{\left.\widetilde{\alpha}_{j}+\widetilde{\kappa}_{\widetilde{\alpha}_{j}^{(1)}}\right)\left(\widetilde{\alpha}_{j}, \xi\right)}} \\
& \times\left(q_{j}^{2} \widetilde{a}_{j} q^{-\left(\widetilde{\alpha}_{j}, \xi\right)}, q_{j}^{2} \widetilde{b}_{j} q^{-\left(\widetilde{\alpha}_{j}, \xi\right)}, \widetilde{c}_{j} q^{-\left(\widetilde{\alpha}_{j}, \xi\right)}, \tilde{d}_{j} q^{-\left(\widetilde{\alpha}_{j}, \xi\right)} ; q_{j}^{2}\right)_{\infty} .
\end{aligned}
$$

Hence $G(\mathbf{z}, \xi)$ is regular at $\xi=\gamma$.

Returning to the second term in the right hand side of (3.21), we next observe that

$$
\begin{aligned}
& \widetilde{\mathcal{S}}\left(s_{i^{*}} \tau_{2} \xi\right) A_{\tau_{2}^{-1} s_{i} *}^{u n n}(\xi) \Phi\left(\mathbf{z}, s_{i} * \tau_{2} \xi\right) \\
& \quad=\frac{\mathcal{W}\left(\mathbf{z}, s_{i} * \tau_{2} \xi\right)}{\mathcal{S}(\mathbf{z})} A_{s_{j}}^{u n n}(\xi)\left(A_{\tau_{2}^{-1}}^{u n n}\left(s_{j} \xi\right) \sum_{\mu \in Q_{+}} \Gamma_{\mu}\left(s_{i} * \tau_{2} \xi\right) q^{-(\mu, \mathbf{z})}\right)
\end{aligned}
$$

since

$$
A_{\tau_{2}^{-1} s_{i} *}^{u n n}(\xi)=A_{s_{j} \tau_{2}^{-1}}^{u n n}(\xi)=A_{s_{j}}^{u n n}(\xi) A_{\tau_{2}^{-1}}^{u n n}\left(s_{j} \xi\right)
$$

by (3.4). Now $\phi_{I, \gamma}^{\mathcal{V}} \circ A_{s_{j}}^{u n n}(\gamma): \mathcal{V} \rightarrow M_{I}(\gamma)$ is the zero map (see the proof of Proposition 3.4), hence we conclude that

$$
\phi_{I, \gamma}^{\mathcal{V}}\left(\widetilde{\mathcal{S}}\left(s_{i *} \tau_{2} \gamma\right) A_{\tau_{2}^{-1} s_{i *}^{*}}^{u n n}(\gamma) \Phi\left(\mathbf{z}, s_{i *} \tau_{2} \gamma\right)\right) \equiv 0
$$

as $M_{I}(\gamma)$-valued holomorphic function in $\mathbf{z} \in E_{\mathbb{C}}$. 
Thus specialising $\xi$ to $\gamma$ in (3.21) and applying the map $\phi_{I, \gamma}^{\mathcal{V}}$ we obtain

$$
C_{s_{i}}^{M_{I}(\gamma)}(\mathbf{z}) \Phi_{\tau_{2}^{-1}}^{I}\left(s_{i} \mathbf{z}, \gamma\right)=n_{\tau_{2}, \tau_{2}}^{s_{i}}(\mathbf{z}, \gamma) \Phi_{\tau_{2}^{-1}}^{I}(\mathbf{z}, \gamma)
$$

It thus remains to show that

$$
n_{\tau_{2}, \tau_{2}}^{s_{i}}(\mathbf{z}, \gamma) \equiv 1
$$

as meromorphic function in $\mathbf{z} \in E_{\mathbb{C}}$. To prove (3.24) first note that

$$
n_{\tau_{2}, \tau_{2}}^{s_{i}}(\mathbf{z}, \gamma)=\frac{\mathfrak{e}_{\alpha_{i}}\left(\left(\alpha_{i}, \mathbf{z}\right),\left(\widetilde{\alpha}_{j}, \gamma\right)\right)-\widetilde{\mathfrak{e}}_{\alpha_{i}}\left(\left(\widetilde{\alpha}_{j}, \gamma\right),\left(\alpha_{i}, \mathbf{z}\right)\right)}{\widetilde{\mathfrak{e}}_{\alpha_{i}}\left(\left(\widetilde{\alpha}_{j}, \gamma\right),-\left(\alpha_{i}, \mathbf{z}\right)\right)}
$$

since $\alpha_{j}=\tau_{2}^{-1}\left(\alpha_{i *}\right)$. But $j \in I$, hence $\gamma \in E_{\mathbb{C}, I}^{\kappa}$ and $\alpha_{i} \in W_{0}\left(\alpha_{j}\right)$ implies that

$$
\left(\widetilde{\alpha}_{j}, \gamma\right)=\widetilde{\kappa}_{\widetilde{\alpha}_{j}}+\widetilde{\kappa}_{2} \widetilde{\alpha}_{j}=\widetilde{\kappa}_{\widetilde{\alpha}_{i}}+\widetilde{\kappa}_{2} \widetilde{\alpha}_{i} .
$$

Consequently

$$
n_{\tau_{2}, \tau_{2}}^{s_{i}}(\mathbf{z}, \gamma)=\frac{\mathfrak{e}_{\alpha_{i}}\left(\left(\alpha_{i}, \mathbf{z}\right), \widetilde{\kappa}_{\widetilde{\alpha}_{i}}+\widetilde{\kappa}_{2 \widetilde{\alpha}_{i}}\right)-\widetilde{\mathfrak{e}}_{\alpha_{i}}\left(\widetilde{\kappa}_{\widetilde{\alpha}_{i}}+\widetilde{\kappa}_{2} \widetilde{\alpha}_{i},\left(\alpha_{i}, \mathbf{z}\right)\right)}{\widetilde{\mathfrak{e}}_{\alpha_{i}}\left(\widetilde{\kappa}_{\widetilde{\alpha}_{i}}+\widetilde{\kappa}_{2} \widetilde{\alpha}_{i},-\left(\alpha_{i}, \mathbf{z}\right)\right)} .
$$

By a direct computation the numerator in (3.25) can be rewritten as

$$
\begin{aligned}
& \mathfrak{e}_{\alpha_{i}}\left(\left(\alpha_{i}, \mathbf{z}\right), \widetilde{\kappa}_{\widetilde{\alpha}_{i}}+\widetilde{\kappa}_{2} \widetilde{\alpha}_{i}\right)-\widetilde{\mathfrak{e}}_{\alpha_{i}}\left(\widetilde{\kappa}_{\widetilde{\alpha}_{i}}+\widetilde{\kappa}_{2} \widetilde{\alpha}_{i},\left(\alpha_{i}, \mathbf{z}\right)\right) \\
& \quad=\frac{\theta\left(\widetilde{d}_{i} / \widetilde{a}_{i}, q^{2\left(\alpha_{i}, \mathbf{z}\right)}, \widetilde{a}_{i} \widetilde{b}_{i}, \widetilde{a}_{i} \widetilde{c}_{i} ; q_{i}^{2}\right)-\theta\left(a_{i} q^{\left(\alpha_{i}, \mathbf{z}\right)}, b_{i} q^{\left(\alpha_{i}, \mathbf{z}\right)}, c_{i} q^{\left(\alpha_{i}, \mathbf{z}\right)}, \widetilde{d}_{i} q^{\left(\alpha_{i}, \mathbf{z}\right)} / a_{i} \widetilde{a}_{i} ; q_{i}^{2}\right)}{\theta\left(q^{2\left(\alpha_{i}, \mathbf{z}\right)}, \widetilde{d}_{i} / \widetilde{a}_{i} ; q_{i}^{2}\right)} .
\end{aligned}
$$

Recall the well known theta function identity

$$
\theta(x \nu, x / \nu, \lambda \mu, \mu / \lambda ; q)-\theta(x \lambda, x / \lambda, \mu \nu, \mu / \nu ; q)=-\frac{\mu}{\lambda} \theta(x \mu, x / \mu, \lambda \nu, \lambda / \nu ; q) .
$$

For an exhaustive discussion of (3.27) and its origin, see [47]. Using (3.27) with $(q, x, v, \lambda, \mu)$ specialised to

$$
\left(q_{i}^{2}, c_{i} \sqrt{a_{i} b_{i}}, \sqrt{a_{i}^{-1} b_{i}}, \sqrt{a_{i} b_{i}} q^{-\left(\alpha_{i}, \mathbf{z}\right)}, \sqrt{a_{i} b_{i}} q^{\left(\alpha_{i}, \mathbf{z}\right)}\right)
$$

we can rewrite $(3.26)$ as

$$
\begin{aligned}
& \mathfrak{e}_{\alpha_{i}}\left(\left(\alpha_{i}, \mathbf{z}\right), \widetilde{\kappa}_{\widetilde{\alpha}_{i}}+\widetilde{\kappa}_{2} \widetilde{\alpha}_{i}\right)-\widetilde{\mathfrak{e}}_{\alpha_{i}}\left(\widetilde{\kappa}_{\widetilde{\alpha}_{i}}+\widetilde{\kappa}_{2} \widetilde{\alpha}_{i},\left(\alpha_{i}, \mathbf{z}\right)\right) \\
& \quad=-\frac{\theta\left(a_{i} q^{-\left(\alpha_{i}, \mathbf{z}\right)}, b_{i} q^{-\left(\alpha_{i}, \mathbf{z}\right)}, c_{i} q^{-\left(\alpha_{i}, \mathbf{z}\right)} \widetilde{d}_{i} q^{-\left(\alpha_{i}, \mathbf{z}\right)} / a_{i} \widetilde{a}_{i} ; q_{i}^{2}\right)}{\theta\left(q^{2\left(\alpha_{i}, \mathbf{z}\right)}, \widetilde{d}_{i} / \widetilde{a}_{i} ; q_{i}^{2}\right)} q^{2\left(\alpha_{i}, \mathbf{z}\right)} .
\end{aligned}
$$

A direct computation shows that the right hand side of (3.28) equals

$$
\widetilde{\mathfrak{e}}_{\alpha_{i}}\left(\widetilde{\kappa}_{\widetilde{\alpha}_{i}}+\widetilde{\kappa}_{2} \widetilde{\alpha}_{i},-\left(\alpha_{i}, \mathbf{z}\right)\right) \text {. }
$$

Hence

$$
n_{\tau_{2}, \tau_{2}}^{s_{i}}(\mathbf{z}, \gamma) \equiv 1
$$

as meromorphic function in $\mathbf{z} \in E_{\mathbb{C}}$, as desired. 
3.6. The link to the spin- $\frac{1}{2} X X Z$ boundary $q K Z$ equations. We specialise the results of the previous subsection to the initial data $(D, \kappa)$ corresponding to the Koornwinder (or $\left.C^{\vee} C_{n}\right)$ case of the Cherednik-Macdonald theory. The five tuple $D=\left(R_{0}, \Delta_{0}, \bullet, \Lambda, \widetilde{\Lambda}\right)$ is then given as follows (we choose our notations to ensure that it matches with the notations in Sect. 2.1). Take $E=\mathbb{R}^{n}(n \geq 2)$ with the standard orthonormal basis $\left\{e_{i}\right\}_{i=1}^{n}$. The root system $R_{0}$ is taken to be the root system of type $B_{n}$ realised by

$$
R_{0}=\left\{ \pm\left(e_{i} \pm e_{j}\right)\right\}_{1 \leq i<j \leq n} \cup\left\{ \pm e_{i}\right\}_{i=1}^{n} .
$$

As ordered basis we take

$$
\Delta_{0}=\left(\alpha_{1}, \ldots, \alpha_{n-1}, \alpha_{n}\right)=\left(e_{1}-e_{2}, \ldots, e_{n-1}-e_{n}, e_{n}\right) .
$$

We are considering the twisted theory $\bullet=t$, hence $\mu_{\alpha}=|\alpha|^{2} / 2$ and $\widetilde{\alpha}=\alpha$ for all $\alpha \in R_{0}$. The lattices are taken to be as small as possible,

$$
\Lambda=\mathbb{Z}^{n}=\widetilde{\Lambda}
$$

(the root lattice of $R_{0}$ ). The affine simple root is $\alpha=\frac{c}{2}-e_{1}$. The Weyl group $W_{0}=$ $\left\langle s_{1}, \ldots, s_{n-1}, s_{n}\right\rangle$ is isomorphic to the hyperoctahedral group $S_{n} \ltimes( \pm 1)^{n}$. Furthermore we have $s_{0}=\tau\left(e_{1}\right) s_{e_{1}}$ and $W=\left\langle s_{0}, \ldots, s_{n}\right\rangle \simeq W_{0} \ltimes \mathbb{Z}^{n}$.

The affine root system $R(D)$ associated to $D$ has five $W$-orbits

$$
W \alpha_{0}, W\left(2 \alpha_{0}\right), W \alpha_{1}, W \alpha_{n}, W\left(2 \alpha_{n}\right) .
$$

A multiplicity function $\kappa: R(D) \rightarrow \mathbb{R}$ thus is determined by five values, which we denote by

$$
\left(\zeta, v, \zeta^{\prime}, v^{\prime}, \kappa\right):=\left(\kappa_{\alpha_{n}}, \kappa_{2 \alpha_{n}}, \kappa_{\alpha_{0}}, \kappa_{2 \alpha_{0}}, \kappa_{\alpha_{1}}\right)
$$

The dual parameters are then obtained by interchanging $v$ and $\zeta^{\prime}$. The Askey-Wilson parameters $\left\{a_{\alpha}, b_{\alpha}, c_{\alpha}, d_{\alpha}\right\}$ are $\{a, b, c, d\}$ (see (2.7)) if $\alpha \in R_{0}$ is short. If $\alpha \in R_{0}$ is long then $\left\{a_{\alpha}, b_{\alpha}, c_{\alpha}, d_{\alpha}\right\}=\left\{q^{2 \kappa},-1, q^{1+2 \kappa},-q\right\}$.

We take $I:=\{1, \ldots, n-1\}$, so that

$$
W_{0, I}=\left\langle s_{1}, \ldots, s_{n-1}\right\rangle \simeq S_{n} .
$$

Note that

$$
E_{\mathbb{C}, I}^{\kappa}=\{(\xi+(n-1) \kappa, \xi+(n-3) \kappa, \ldots, \xi+(1-n) \kappa) \mid \xi \in \mathbb{C}\} .
$$

Recall the spin representation $\left(\pi_{(\xi)}^{s p},\left(\mathbb{C}^{2}\right)^{\otimes n}\right.$ ) (see Proposition 2.1) and the elements $w_{\underline{\epsilon}} \in W_{0}\left(\underline{\epsilon} \in\{ \pm 1\}^{n}\right)$ defined by (2.2), which are the minimal coset representatives $W_{0}^{I}$ of $W_{0} / W_{0, I}$ (see Lemma 2.3). By [65, Prop. 3.5] we have

$$
\left(\pi_{\gamma}^{I}, M_{I}(\gamma)\right) \simeq\left(\pi_{(\xi)}^{s p},\left(\mathbb{C}^{2}\right)^{\otimes n}\right)
$$

as $H(\kappa)$-modules, with $\gamma \in E_{\mathbb{C}, I}^{\kappa}$ given by

$$
\gamma:=(\xi+(n-1) \kappa, \xi+(n-3) \kappa, \ldots, \xi+(1-n) \kappa) .
$$

The isomorphism $M_{I}(\gamma) \stackrel{\sim}{\longrightarrow}\left(\mathbb{C}^{2}\right)^{\otimes n}$ of $H(\kappa)$-modules maps the basis element $v_{w_{\underline{\epsilon}}}(\gamma) \in M_{I}(\gamma)$ to $v_{\underline{\epsilon}} \in\left(\mathbb{C}^{2}\right)^{\otimes n}$ for all $\underline{\epsilon} \in\{ \pm 1\}^{n}$ (see Lemma 2.3).

In the remainder of this subsection we assume that $\gamma \in E_{\mathbb{C}, I}^{\kappa}$ (see (3.32)) is generic and satisfies (3.18) (this gives generic conditions on $\xi$ and $\kappa$ ). The isomorphism $M_{I}(\gamma) \stackrel{\sim}{\longrightarrow}\left(\mathbb{C}^{2}\right)^{\otimes n}$ allows to transfer the definitions and results of this section to the setting of the XXZ spin chain (see Sect. 2) in the following way.

Let $\underline{\epsilon}, \underline{\epsilon^{\prime}} \in\{ \pm 1\}^{n}$ and $\sigma \in W_{0}$, then 
(1) The vector $b_{w_{\epsilon}^{-1}}^{I}(\gamma) \in M_{I}(\gamma)$ (Corollary 3.6 and Proposition 3.4) is mapped to $b_{\epsilon} \in\left(\mathbb{C}^{2}\right)^{\otimes n}($ Lemma 2.4);

(2) The quantum affine $\mathrm{KZ}$ equations associated to $M_{I}(\gamma)$ (Definition 3.8) become the boundary quantum KZ equations (2.6) associated to the XXZ spin- $\frac{1}{2}$ chain;

(3) The power series solution $\Phi_{w_{\epsilon}^{-1}}^{I}(\cdot, \gamma)$ (Proposition 3.13) of the quantum affine $\mathrm{KZ}$ equations become the power series solution $\Phi_{\epsilon}(\cdot)$ (Theorem 2.7) of the boundary qKZ equation (2.6). In particular, Theorem 2.7 is a special case of Proposition 3.13.

(4) The connection coefficient $m_{w_{\underline{\epsilon}}, w_{\epsilon^{\prime}}}^{I, \sigma}(\cdot, \gamma)$ (Sect. 3.5) equals $M_{c m ; \underline{\epsilon}, \underline{\epsilon}^{\prime}}^{\sigma}(\cdot, \xi)$ (Sect. 2.4).

We are now ready to prove Theorem 2.10 as a consequence of the explicit expressions of the connection matrix of the quantum affine $\mathrm{KZ}$ equation for principal series modules (see Theorem 3.15).

Proof of Theorem 2.10. Recall that $M_{c m}^{\sigma}(\mathbf{z}, \xi)$ is the linear operator on $\left(\mathbb{C}^{2}\right)^{\otimes n}$ defined by

$$
\begin{aligned}
M_{c m}^{\sigma}(\mathbf{z}, \xi) v_{\underline{\epsilon}^{\prime}} & :=\sum_{\underline{\epsilon} \in\{ \pm 1\}^{n}} M_{c m ; \underline{\epsilon}, \underline{\epsilon}^{\prime}}^{\sigma}(\mathbf{z}, \xi) v_{\underline{\epsilon}} \\
& =\sum_{\underline{\epsilon} \in\{ \pm 1\}^{n}} m_{w_{\underline{\epsilon}}, w_{\underline{\epsilon^{\prime}}}}^{I, \sigma}(\mathbf{z}, \gamma) v_{\underline{\epsilon}} .
\end{aligned}
$$

Fix $i \in\{1, \ldots, n\}$ and note that $i^{*}=i$. By Theorem 3.15 we have

$$
M_{c m}^{s_{i}}(\mathbf{z}, \xi) v_{\underline{\epsilon}^{\prime}}=v_{\underline{\epsilon}^{\prime}} \quad \text { if } s_{i} w_{\underline{\epsilon}^{\prime}} \notin W_{0}^{I},
$$

and

$$
M_{c m}^{s_{i}}(\mathbf{z}, \xi) v_{\underline{\epsilon}^{\prime}}=m_{w_{\underline{\epsilon}^{\prime}}, w_{\underline{\epsilon}^{\prime}}}^{I, s_{i}}(\mathbf{z}, \gamma) v_{\underline{\epsilon}^{\prime}}+m_{w_{\underline{\epsilon}^{\prime \prime}}, w_{\underline{\epsilon}^{\prime}}}^{I, s_{i}}(\mathbf{z}, \gamma) v_{\underline{\epsilon}^{\prime \prime}} \quad \text { if } \quad s_{i} w_{\underline{\epsilon}^{\prime}} \in W_{0}^{I},
$$

where $\underline{\epsilon}^{\prime \prime} \in\{ \pm 1\}^{n}$ is such that $s_{i} w_{\epsilon^{\prime}}=w_{\epsilon^{\prime \prime}}$. Let $W_{0} \simeq S_{n} \ltimes( \pm 1)^{n}$ act on $\{ \pm 1\}^{n}$ by permutations and sign changes. We need the following simple lemma.

Lemma 3.17. Let $\underline{\epsilon} \in\{ \pm 1\}^{n}$ and $1 \leq j<n$.

(i) $s_{j} w_{\underline{\epsilon}} \in W_{0}^{I} \Leftrightarrow\left(\epsilon_{j}, \epsilon_{j+1}\right) \in\{(+1,-1),(-1,+1)\}$, in which case $s_{j} w_{\underline{\epsilon}}=w_{s_{j} \underline{\epsilon}}$.

(ii) $s_{n} w_{\underline{\epsilon}}=w_{s_{n} \underline{\epsilon}} \in W_{0}^{I}$.

Proof. (ii) is trivial in view of the explicit expression (2.2) of $w_{\underline{\epsilon}} \in W_{0}^{I}$. The explicit expression (2.2) of $w_{\underline{\epsilon}}$ immediately implies that $s_{j} w_{\underline{\epsilon}}=w_{s_{j} \underline{\epsilon}}$ if $\left(\epsilon_{j}, \epsilon_{j+1}\right) \epsilon$ $\{(+1,-1),(-1,+1)\}$. Suppose that $\left(\epsilon_{j}, \epsilon_{j+1}\right)=(+1,+1)$. Then

$$
s_{j} w_{\underline{\epsilon}}=w_{\underline{\epsilon}} s_{k}
$$

for some $1 \leq k \leq j<n$ since

$$
s_{j}\left(s_{r} s_{r+1} \cdots s_{n-1} s_{n}\right)= \begin{cases}\left(s_{r} s_{r+1} \cdots s_{n-1} s_{n}\right) s_{j} & \text { if } j+1<r \leq n, \\ \left(s_{r} s_{r+1} \cdots s_{n-1} s_{n}\right) s_{j-1} & \text { if } 1 \leq r<j .\end{cases}
$$

Hence $s_{j} w_{\underline{\epsilon}} \notin W_{0}^{I}$.

Suppose that $\left(\epsilon_{j}, \epsilon_{j+1}\right)=(-1,-1)$. Then we need in addition the equality

$$
s_{j}\left(\left(s_{j+1} s_{j+2} \cdots s_{n}\right)\left(s_{j} s_{j+1} \cdots s_{n}\right)\right)=\left(\left(s_{j+1} s_{j+2} \cdots s_{n}\right)\left(s_{j} s_{j+1} \cdots s_{n}\right)\right) s_{n-1}
$$

in $W_{0}$ to conclude that $s_{j} w_{\underline{\epsilon}}=w_{\underline{\epsilon}} s_{k}$ for some $1 \leq k<n$, hence $s_{j} w_{\underline{\epsilon}} \notin W_{0}^{I}$. 
Continuing with the proof of Theorem 2.10, we conclude from (3.33), (3.34), Lemma 3.17 and the explicit expressions for the connection coefficients $m_{w_{\underline{\epsilon}}, w_{\underline{\epsilon}^{\prime}}}^{I, s_{i}}, \gamma$ ) (see Theorem 3.15) that for all $\underline{\epsilon}^{\prime}=\left(\epsilon_{1}^{\prime}, \ldots, \epsilon_{n}^{\prime}\right) \in\{ \pm 1\}^{n}$,

$$
M_{c m}^{s_{i}}(\mathbf{z}, \xi) v_{\underline{\epsilon}^{\prime}}=R^{(i)}\left(\left(\alpha_{i}, \mathbf{z}\right),\left(\alpha_{i}, w_{\underline{\epsilon}^{\prime(i)}} \gamma\right)\right)_{i, i+1} v_{\underline{\epsilon}^{\prime}}
$$

if $1 \leq i<n$ with $\underline{\epsilon}^{\prime(i)}:=\left(\epsilon_{1}^{\prime}, \ldots, \epsilon_{i-1}^{\prime}, 1,-1, \epsilon_{i+1}^{\prime}, \ldots, \epsilon_{n}^{\prime}\right)$ and

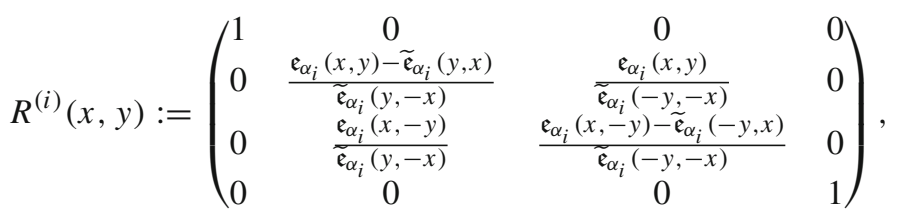

and

$$
M_{c m}^{s_{n}}(\mathbf{z}, \xi) v_{\underline{\epsilon}^{\prime}}=K\left(\left(\alpha_{n}, \mathbf{z}\right),\left(\alpha_{n}, w_{\underline{\epsilon}^{\prime(n)}} \gamma\right)\right)_{n} v_{\underline{\epsilon}^{\prime}}
$$

with $\underline{\epsilon}^{\prime(n)}:=\left(\epsilon_{1}^{\prime}, \ldots, \epsilon_{n-1}^{\prime},+1\right)$ and

$$
K(x, y):=\left(\begin{array}{cc}
\frac{\mathfrak{e}_{\alpha_{n}}(x, y)-\widetilde{\mathfrak{e}}_{\alpha_{n}}(y, x)}{\widetilde{\mathfrak{e}}_{\alpha}(y,-x)} & \frac{\mathfrak{e}_{\alpha_{n}}(x, y)}{\mathfrak{\mathfrak { e }}_{\alpha_{n}}(-y,-x)} \\
\frac{\mathfrak{e}_{\alpha_{n}}(x,-y)}{\mathfrak{e}_{\alpha_{n}}(y,-x)} & \frac{\mathfrak{e}_{\alpha_{n}}(x,-y)-\mathfrak{\mathfrak { e }}_{\alpha_{n}}(-y, x)}{\mathfrak{\mathfrak { E }}_{\alpha_{n}}(-y,-x)}
\end{array}\right) .
$$

If $1 \leq i<n$ then $\left(\mathbb{Z}, \alpha_{i}^{\vee}\right)=\mathbb{Z}$, so [64, (1.9) and Prop. 1.7] gives

$$
\begin{aligned}
\frac{\mathfrak{e}_{\alpha_{i}}(x, y)-\widetilde{\mathfrak{e}}_{\alpha_{i}}(y, x)}{\widetilde{\mathfrak{e}}_{\alpha_{i}}(y,-x)}=\frac{\theta\left(q^{2 \kappa}, q^{y-x} ; q\right)}{\theta\left(q^{y}, q^{2 \kappa-x} ; q\right)} q^{(2 \kappa-y) x}=B_{c m}(x,-y), \\
\frac{\mathfrak{e}_{\alpha_{i}}(x,-y)}{\widetilde{\mathfrak{e}}_{\alpha_{i}}(y,-x)}=\frac{\theta\left(q^{2 \kappa-y}, q^{-x} ; q\right)}{\theta\left(q^{2 \kappa-x}, q^{-y} ; q\right)} q^{2 \kappa(x-y)}=A_{c m}(x, y),
\end{aligned}
$$

hence

$$
M_{c m}^{s_{i}}(\mathbf{z}, \xi) v_{\underline{\epsilon}^{\prime}}=P_{i, i+1} R_{c m}\left(z_{i}-z_{i+1},\left(\alpha_{i}, w_{\underline{\epsilon}^{\prime(i)}} \gamma\right)\right)_{i, i+1} v_{\underline{\epsilon}^{\prime}}
$$

(see Theorem 2.10 for the definitions of $A_{c m}(x, y), B_{c m}(x, y)$ and $R_{c m}(x, y)$ ). For $i=n$ a direct computation using (3.30) gives

$$
\mathfrak{e}_{\alpha_{n}}(x, y)=\mathcal{C}(x, y)
$$

(see (2.9) for the definition of $\mathcal{C}(x, y)$ ), hence

$$
M_{c m}^{s_{n}}(\mathbf{z}, \xi) v_{\underline{\epsilon}^{\prime}}=K_{c m}\left(z_{n},\left(\alpha_{n}, w_{\underline{\epsilon}^{\prime(n)}} \gamma\right)\right)_{n} v_{\underline{\epsilon}^{\prime}}
$$

(see Theorem 2.10 for the definition of $K_{c m}(x, y)$ ). So the following lemma completes the proof of Theorem 2.10 .

Lemma 3.18. Let $1 \leq i<n, \underline{\epsilon} \in\{ \pm 1\}^{n}$ and $\gamma \in E_{\mathbb{C}, I}^{\kappa}($ see (3.32)).

(1) If $\left(\epsilon_{i}, \epsilon_{i+1}\right) \in\{(+1,-1),(-1,+1)\}$ then

$$
\left(\alpha_{i}, w_{\underline{\epsilon}^{(i)}} \gamma\right)=2 \xi-2 \kappa\left(\epsilon_{1}+\epsilon_{2}+\cdots+\epsilon_{i-1}\right) .
$$


(2) $\left(\alpha_{n}, w_{\epsilon^{(n)}} \gamma\right)=\xi-\kappa\left(\epsilon_{1}+\epsilon_{2}+\cdots+\epsilon_{n-1}\right)$.

Proof. Write $V_{\epsilon}:=\left\{j \in\{1, \ldots, n\} \mid \epsilon_{j}=-1\right\}=\left\{j_{1}, \ldots, j_{r}\right\}$ with $j_{1}<j_{2}<\cdots<j_{r}$. (1) Suppose that $\left(\epsilon_{i}, \epsilon_{i+1}\right)=(+1,-1)$ and let $1 \leq t \leq r$ be the index such that $j_{t}=i+1$. Using the expression

$$
w_{\underline{\epsilon}}=\left(s_{j_{r}} s_{j_{r}+1} \cdots s_{n}\right) \cdots\left(s_{j_{2}} s_{j_{2}+1} \cdots s_{n}\right)\left(s_{j_{1}} s_{j_{1}+1} \cdots s_{n}\right)
$$

(see (2.2)) we obtain

$$
w_{\underline{\epsilon}^{(i)}}^{-1}\left(\alpha_{i}\right)=w_{\underline{\epsilon}}^{-1}\left(\alpha_{i}\right)=e_{i-t+1}+e_{n-t+1} .
$$

Then

$$
\begin{aligned}
\left(\alpha_{i}, w_{\epsilon^{(i)}} \gamma\right)=\gamma_{i-t+1}+\gamma_{n-t+1} & =2 \xi+(4 t-2 i-2) \kappa \\
& =2 \xi+2 \kappa(t-1)-2 \kappa(i-t) \\
& =2 \xi-2 \kappa\left(\epsilon_{1}+\cdots+\epsilon_{i-1}\right) .
\end{aligned}
$$

If $\left(\epsilon_{i}, \epsilon_{i+1}\right)=(-1,+1)$ then we apply the result of the previous paragraph to $\underline{\epsilon}^{\prime}:=s_{i} \underline{\epsilon}$ to conclude that

$$
\begin{aligned}
\left(\alpha_{i}, w_{\underline{\epsilon}^{(i)}} \gamma\right) & =\left(\alpha_{i}, w_{\underline{\epsilon}^{\prime}} \gamma\right) \\
& =2 \xi-2 \kappa\left(\epsilon_{1}^{\prime}+\cdots+\epsilon_{i-1}^{\prime}\right) \\
& =2 \xi-2 \kappa\left(\epsilon_{1}+\cdots+\epsilon_{i-1}\right) .
\end{aligned}
$$

(2) Suppose that $\epsilon_{n}=+1$, so that $j_{r}<n$. Then

$$
w_{\underline{\epsilon}^{(n)}}^{-1}\left(\alpha_{n}\right)=w_{\underline{\epsilon}}^{-1}\left(\alpha_{n}\right)=e_{n-r}
$$

hence

$$
\begin{aligned}
\left(\alpha_{n}, w_{\underline{\epsilon}^{(n)}} \gamma\right) & =\gamma_{n-r} \\
& =\xi+\kappa(1-n+2 r)=\xi+\kappa r-\kappa(n-1-r) \\
& =\xi-\kappa\left(\epsilon_{1}+\epsilon_{2}+\cdots+\epsilon_{n-1}\right) .
\end{aligned}
$$

If $\epsilon_{n}=-1$ then we apply this result to $\underline{\epsilon}^{\prime}:=s_{n} \underline{\epsilon}$ to reach the same conclusion,

$$
\begin{aligned}
\left(\alpha_{n}, w_{\underline{\epsilon}^{(n)}} \gamma\right) & =\left(\alpha_{n}, w_{\epsilon^{(n)}} \gamma\right) \\
& =\xi-\kappa\left(\epsilon_{1}^{\prime}+\epsilon_{2}^{\prime}+\cdots+\epsilon_{n-1}^{\prime}\right) \\
& =\xi-\kappa\left(\epsilon_{1}+\epsilon_{2}+\cdots+\epsilon_{n-1}\right) .
\end{aligned}
$$

This completes the proof of the lemma.

Acknowledgements. I thank Ivan Cherednik, Masatoshi Noumi, Nicolai Reshetikhin and Bart Vlaar for discussions and Yasushi Komori for providing me with a copy of [31].

Open Access This article is distributed under the terms of the Creative Commons Attribution 4.0 International License (http://creativecommons.org/licenses/by/4.0/), which permits unrestricted use, distribution, and reproduction in any medium, provided you give appropriate credit to the original author(s) and the source, provide a link to the Creative Commons license, and indicate if changes were made. 


\section{References}

1. Andrews, G.E., Baxter, R.J., Forrester, P.J.: Eight-vertex SOS model and generalized Rogers-Ramanujan type identities. J. Stat. Phys. 35, 193-266 (1984)

2. Avan, J., Ragouchy, E.: A new dynamical reflection algebra and related quantum integrable systems. Lett. Math. Phys. 101, 85-101 (2012)

3. Askey, R., Wilson, J.: Some basic hypergeometric orthogonal polynomials that generalize Jacobi polynomials. Mem. Am. Math. Soc. 54, 319 (1985)

4. Baseilhac, P., Kojima, T.: Correlation functions on the half-infinite XXZ spin chain with a triangular boundary. Nucl. Phys. B 880, 378-413 (2014)

5. Baxter, R.J.: Exactly solved models of statistical mechanics. Academic Press, London (1982)

6. Behrend, R.E., Pearce, P.A.: Solutions of the boundary Yang-Baxter equation for A-D-E models. Int. J. Mod. Phys. B 11, 2833-2847 (1997)

7. Behrend, R.E., Pearce, P.A., O'Brien, D.L.: Interaction-round-a-face models with fixed boundary conditions: the ABF fusion hierarchy. J. Statist. Phys. 84(1-2), 1-48 (1996)

8. Chalykh, O., Etingof, P.: Orthogonality relations and Cherednik identities for multivariable BakerAkhiezer functions. Adv. Math. 238, 246-289 (2013)

9. Cherednik, I.: Double affine Hecke algebras. London Math. Soc. Lecture Note Series 319. Cambridge University Press, Cambridge (2005)

10. Cherednik, I.: Quantum Knizhnik-Zamolodchikov equations and affine root systems. Comm. Math. Phys. 150, 109-136 (1992)

11. Cherednik, I.: Induced representations of double affine Hecke algebras and applications. Math. Res. Lett. 1, 319-337 (1994)

12. Date, E., Jimbo, M., Miwa, T., Okado, M.: Fusion of the eight vertex SOS model. Lett. Math. Phys. 12(3), 209-215 (1986)

13. Delius, G.W., MacKay, N.J.: Quantum group symmetry in sine-Gordon and affine Toda field theories on the half line. Comm. Math. Phys. 233, 173-190 (1995)

14. Diejen, J.-F.van : Integrability of difference Calogero-Moser systems. J. Math. Phys. 35, 2983-3004 (1994)

15. Etingof, P.I., Frenkel, I.B., Kirillov A.A. Jr.: Lectures on representation theory and KnizhnikZamolodchikov equations. In: Math. Surveys and Monographs, vol. 58. American Mathematical Society, New York (1998)

16. Etingof, P., Varchenko, A.: Solutions of the quantum dynamical Yang-Baxter equation and dynamical quantum groups. Comm. Math. Phys. 196, 591-640 (1998)

17. Etingof, P., Varchenko, A.: Exchange dynamical quantum groups. Comm. Math. Phys. 205(1), 19-52 (1999)

18. Fan, H., Hou, B.-Y., Shi, K.-J.: General solution of reflection equation for eight-vertex SOS model. J. Phys. A 28, 4743-4749 (1995)

19. Felder, G.: Conformal field theory and integrable systems associated to elliptic curves. In: Proceedings of the International Congress of Mathematicians, vol. 2, pp. 1247-1255. Zürich (1994)

20. G. Felder, Elliptic quantum groups. XIth International Congress of Mathematical Physics (Paris 1994), pp. 211-218. Int. Press, Cambridge (1995)

21. Felder, G., Tarasov, V., Varchenko, A.: Monodromy of solutions of the elliptic quantum KnizhnikZamolodchikov-Bernard difference equations. Int. J. Math. 10(8), 943-975 (1999)

22. Felder, G., Varchenko, A.: Algebraic Bethe ansatz for the elliptic quantum group $E_{\tau, \eta}\left(s l_{2}\right)$. Nucl. Phys. B 480, 485-503 (1996)

23. Filali, G.: Elliptic dynamical reflection algebra and partition function of SOS model with reflecting end. J. Geom. Phys. 61(10), 1789-1796 (2011)

24. Filali, G., Kitanine, N.: Spin chains with non-diagonal boundaries and trigonometric SOS model with reflecting end. SIGMA 7, paper 012, p. 22 (2011)

25. Di Francesco, P.: Boundary qKZ equation and generalized Razumov-Stroganov sum rules for open IRF models. J. Stat. Mech. Theory Exp. no. 11, P11003, p. 18 (2005)

26. Di Francesco, P., Zinn-Justin, P.: Quantum Knizhnik-Zamolodchikov equation: reflecting boundary conditions and combinatorics. J. Stat. Mech. Theory Exp. no. 12, P12009, p. 30 (2007)

27. Frenkel, I., Reshetikhin, N.: Quantum affine algebras and holonomic difference equations. Comm. Math. Phys. 146, 1-60 (1992)

28. Gervais, J.-L., Neveu, A.: Novel triangle relation and absence of tachyons in Liouville string field theory. Nucl. Phys. B 238(1), 125-141 (1984)

29. de Gier, J., Nichols, A.: The two-boundary Temperley-Lieb algebra. J. Algebra 321(4), 1132-1167 (2009)

30. de Gier, J., Pyatov, P.: Factorized solutions of Temperley-Lieb qKZ equations on a segment. Adv. Theor. Math. Phys. 14(3), 795-877 (2010) 
31. Hikami, K., Komori, Y.: Boundary Boltzmann weight for the eight-vertex SOS model: vertex-IRF correspondence. J. Phys. Soc. Jpn. 67(1), 78-82 (1998)

32. Hou, B.-Y., Shi, K.-J., Fan, H., Yang, Z.-X.: Solution of reflection equation. Comm. Theor. Phys. 23(2), 163-166 (1995)

33. Inami, T., Konno, H.: Integrable XYZ spin chain with boundaries. J. Phys. A 27(24), L913-L918 (1994)

34. Jimbo, M., Miwa, T.: Algebraic analysis of solvable lattice models. In: Proceedings of Regional Conference Series in Mathematics no. 85, AMS (1995)

35. Jimbo, M., Kedem, R., Konno, H., Miwa, T., Weston, R.: Difference equations in spin chains with a boundary. Nucl. Phys. B 448, 429-456 (1995)

36. Jimbo, M., Kuniba, A., Miwa, T., Okado, M.: The $A_{n}^{(1)}$ face models. Comm. Math. Phys. 119, 543-565 (1988)

37. Jimbo, M., Miwa, T., Okado, M.: Solvable lattice models related to the vector representation of classical simple Lie algebras. Comm. Math. Phys. 116, 507-525 (1988)

38. Kasatani, M.: Boundary quantum Knizhnik-Zamolodchikov equations. In: Proceedings of New trends in quantum integrable systems. pp. 157-171, World Sci. Publ., Hackensack (2011)

39. Koelink, E., Stokman, J.V.: The Askey-Wilson function transform. Int. Math. Res. Not. IMRN 2001(22), 1203-1227 (2001)

40. Kojima, T.: Finite XXZ critical chain with double boundaries. arXiv:nlin/0008014

41. Kolb, S.: Quantum symmetric Kac-Moody pairs. Adv. Math. 267, 395-469 (2014)

42. Komori, Y., Hikami, K.: Quantum integrability of the generalized elliptic Ruijsenaars models. J. Phys. A Math. Gen. 30, 4341-4364 (1997)

43. Komori, Y., Hikami, K.: Elliptic $K$-matrix associated with Belavin's symmetric $R$-matrix. Nucl. Phys. B 494, 687-701 (1997)

44. Konno, H.: Dynamical R matrices of elliptic quantum groups and connection matrices for the $q$-KZ equations. SIGMA Symmetry Integrability Geom. Methods Appl. vol. 2, paper 091, p. 25 (2006)

45. Konno, H.: Fusion of Baxter's elliptic R-matrix and the vertex-face correspondence. Ann. Henri Poincaré 7(7-8), 1555-1567 (2006)

46. Koornwinder, T.H.: Askey-Wilson polynomials for root systems of type BC. Contemp. Math. 139, 189204 (1992)

47. Koornwinder, T.H.: On the equivalence of two fundamental theta identities. Anal. Appl. (Singap) 12, 711725 (2014)

48. Kulish, P.P.: Yang-Baxter equation and reflection equations in integrable models. In: "Low-dimensional models in statistical physics and quantum field theory", pp. 125-144, Lecture Notes in Physics, no. 469, Springer (1996)

49. Lusztig, G.: Affine Hecke algebras and their graded version. J. Am. Math. Soc. 2(3), 599-635 (1989)

50. Meer, M.van : Bispectral quantum Knizhnik-Zamolodchikov equations for arbitrary root systems. Selecta Math. (N.S.) 17(1), 183-221 (2011)

51. van Meer, M., Stokman, J.V.: Double affine Hecke algebras and bispectral quantum KnizhnikZamolodchikov equations. Int. Math. Res. Not. IMRN 2010(6), 969-1040 (2010)

52. Moura, A.A.: Elliptic dynamical R-matrices from the monodromy of the $q$-Knizhnik-Zamolodchikov equations for the standard representation of $U_{q}\left(\widetilde{\mathfrak{s l}}_{n+1}\right)$. Asian J. Math. 7(1), 91-114 (2003)

53. Rains, E.: $B C_{n}$-symmetric Abelian functions. Duke Math. J. 135(1), 99-180 (2006)

54. Reshetikhin, N.: Jackson-type integrals, Bethe vectors, and solutions to a difference analog of the Knizhnik-Zamolodchikov system. Lett. Math. Phys. 26, 153-165 (1992)

55. Reshetikhin, N., Stokman, J.V., Vlaar, B.H.M.: Boundary quantum Knizhnik-Zamolodchikov equations and Bethe vectors. Comm. Math. Phys. 336(2), 953-986 (2015)

56. Rosengren, H.: An Izergin-Korepin-type identity for the 8vSOS model, with applications to alternating sign matrices. Adv. in Appl. Math. 43(2), 137-155 (2009)

57. Ruijsenaars, S.N.M.: Complete integrability of relativistic Calogero-Moser systems and elliptic function identities. Comm. Math. Phys. 110, 191-213 (1987)

58. Sahi, S.: Nonsymmetric Koornwinder polynomials and duality. Ann. Math. (2) 150( 1), 267-282 (1999)

59. Sklyanin, E.K.: Boundary conditions for integrable quantum systems. J. Phys. A Math. Gen. 21, 23752389 (1988)

60. Smirnov, F.: General formula for soliton form factors in sine-Gordon model. J. Phys. A 19(10), L575L578 (1986)

61. Stokman, J.V.: Some remarks on very-well-poised ${ }_{8} \phi_{7}$ series. SIGMA Symmetry Integrability Geom. Methods Appl. vol. 8, paper 039, p. 17 (2012)

62. Stokman, J.V.: Quantum affine Knizhnik-Zamolodchikov equations and quantum spherical functions, I. Int. Math. Res. Not. IMRN 2011(5), 1023-1090 (2011)

63. Stokman, J.V.: The $c$-function expansion of a basic hypergeometric function associated to root systems. Ann. Math. (2) 179(1), 253-299 (2014)

64. Stokman, J.V.: Connection coefficients for basic Harish-Chandra series. Adv. Math. 250, 351-386 (2014) 
65. Stokman, J.V., Vlaar, B.H.M.: Koornwinder polynomials and the XXZ spin chain. J. Approx. Theory (to appear). arXiv: 1310.5545

66. Stokman, J.V.: Macdonald-Koornwinder polynomials. chapter for Volume 5 of the Askey-Bateman project (to appear). arXiv:1111.6112

67. de Vega, H.J., González-Ruiz, A.: Boundary $K$-matrices for the XYZ, XXZ and XXX spin chains. J. Phys. A Math. Gen. 27, 6129-6137 (1994)

68. Tarasov, V., Varchenko, A.: Geometry of $q$-hypergeometric functions, quantum affine algebras and elliptic quantum groups. Astérisque 246, 1-135 (1997)

69. Weston, R.: Correlation functions and the boundary qKZ equation in a fractured XXZ chain. J. Stat. Mech. Theory Exp. (12) P12002, p. 28 (2011)

Communicated by N. Reshetikhin 